\title{
Realizing IR theories by projections in the UV
}

\section{Fabian Klos ${ }^{a}$ and Daniel Roggenkamp ${ }^{b}$}

${ }^{a}$ Institut für Theoretische Physik, Universität Heidelberg, Philosophenweg 19, 69120 Heidelberg, Germany

${ }^{b}$ Institut für Mathematik, Universität Mannheim, B6, 26, 68131 Mannheim, Germany

E-mail: F.Klos@ThPhys.Uni-Heidelberg.de, roggenkamp@uni-mannheim.de

Abstract: We show how (topologically twisted) quantum field theories in the IR of bulk RG flows can be represented within the respective UV theories by means of codimenionone projection defects. Indeed, from this perspective, RG flows of bulk theories can be described in terms of RG flows of the codimension-one identity defect in the fixed UV bulk theory. We illustrate this in the example of RG flows between supersymmetric LandauGinzburg orbifold models, for which the respective defects can be described in terms of matrix factorizations.

Keywords: Field Theories in Lower Dimensions, Renormalization Group, Topological Field Theories

ArXiv EPrint: 1907.12339 


\section{Contents}

1 Introduction 1

2 Defects in 2d TQFTs 5

3 RG-networks in 2d TQFTs $\quad 7$

3.1 Projections from RG defects $\quad 7$

\begin{tabular}{ll}
3.2 & Representing the IR in the UV \\
\hline
\end{tabular}

$\begin{array}{lll}3.3 & \text { Bulk RG flow as defect flow } & 15\end{array}$

$\begin{array}{lll}3.4 & \text { IR theories from projections } & 15\end{array}$

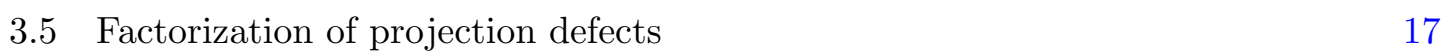

$\begin{array}{ll}3.6 & \text { Relation to the generalized orbifold procedure } \\ \end{array}$

4 RG-networks in Landau-Ginzburg orbifolds $\quad 21$

4.1 B-type defects in Landau-Ginzburg models 21

4.2 Defects $\mathcal{M}_{d} / \mathbb{Z}_{d} \rightarrow \mathcal{M}_{d^{\prime}} / \mathbb{Z}_{d^{\prime}}$

4.3 RG defects in LG orbifolds 25

4.4 Representing $\mathcal{M}_{d^{\prime}} / \mathbb{Z}_{d^{\prime}}$ in $\mathcal{M}_{d} / \mathbb{Z}_{d}$ for $d^{\prime}<d \quad 26$

4.5 The limit $d \rightarrow \infty \quad 28$

$\begin{array}{llr}5 & \text { Conclusion } & 29\end{array}$

$\begin{array}{ll}\text { A Properties of projection defects } & 31\end{array}$

A.1 IR bulk fields in the UV 31

A.2 Bimodule equal bicomodule morphisms $\quad 32$

A.3 $P$-modules $B$ and $B \otimes P \cong B \quad 32$

A.4 $P$-adjunction 33

A.5 Projections with unit and counit 34

A.6 Equivalence of (co)multiplication and Frobenius properties for projections $\quad 35$

A.7 Adjoints of induced RG defects 36

$\begin{array}{ll}\text { B Generalized orbifold theories } & 37\end{array}$

C Orbifold minimal models as generalized orbifolds $\quad 40$

$\begin{array}{lll}\text { C.1 Orbifold identity defect } & 40\end{array}$

$\begin{array}{lll}\text { C.2 Nakayama automorphism } & 41\end{array}$

$\begin{array}{lll}\text { C.3 Bulk space } & 42\end{array}$

C.4 Defects and their adjoints 42

C.5 Left boundary conditions and their adjoints 43

C.6 Equivariant generators of the orbifold identity defect 43

C.7 Important calculations 44

C.7.1 Comultiplication of identity defect $A \quad 44$

$\begin{array}{lll}\text { C.8 (Co)evaluation maps } & 48\end{array}$ 
D Explicit calculations for RG defects in LG orbifolds

D.1 $R \otimes_{A} R^{*} \cong A \quad 51$

D.2 The projection defect $P \quad 52$

D.3 Boundary conditions satisfying $B \otimes_{A} P \cong B \quad 53$

D.4 IR symmetry defects in the UV 54

D.5 $R_{\infty} \otimes_{\mathrm{U}(1)} R_{\infty}^{*} \cong I_{\mathrm{IR}}$

\section{Introduction}

In quantum field theories, the renormalization group $(\mathrm{RG})$ flow drives theories at high ultraviolet (UV) energies to theories at low infrared (IR) energy. In this paper, we describe a method to construct IR correlators directly within the UV theory, by inserting certain codimension-one defects into UV correlators. This allows us to represent the entire IR theory in terms of the UV theory.

While this approach might also be useful in more general situations, in this paper we will only deal with quantum field theories admitting a topological twist compatible with the RG flow. The topological twist provides a good handle on defects allowing us to relate the twisted theories in the IR and UV of RG flows. For brevity and concreteness we will restrict our discussion to two-dimensional quantum field theories, such as $2 d N=(2,2)$ supersymmetric QFTs, but we expect our method to be applicable in any dimension.

Starting point of the construction are RG defects as introduced in [1]. These are domain walls between UV and IR theories obtained in the following way. Consider a perturbation of a scale invariant quantum field theory by a relevant local operator. The RG flow drives the theory from the original theory in the UV to some other theory in the IR. If the perturbation is restricted to a finite region of space-time, the $R G$ flow drives the theory to the IR on the domain of the perturbation, while leaving it at the UV on the rest of space-time. Along the way, it creates a domain wall $R$ on the boundary of the perturbation domain, separating the IR theory from the UV theory:

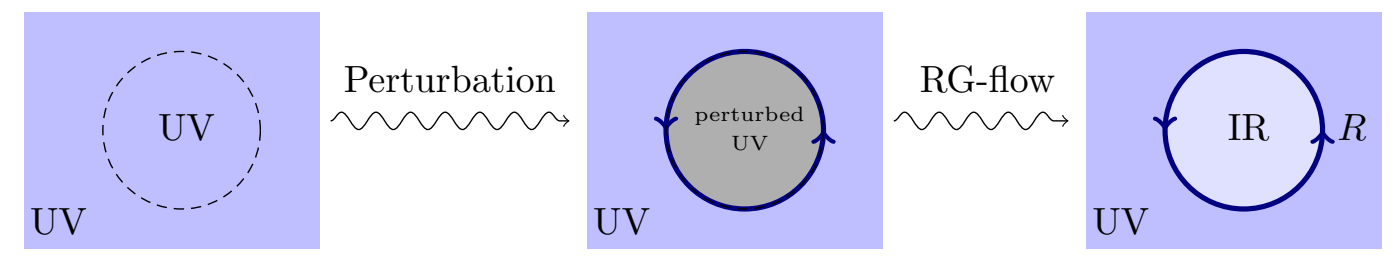

The $R G$ defects $R$ obtained in this way capture the entire relation between UV and IR theories. They project UV degrees of freedom onto the IR theory and embed IR degrees of freedom into the UV theory.

In order to get a good handle on defect lines, in particular the behavior of correlation functions under changes of their position, we now pass to the topologically twisted theory. Compatibility of the RG flow with the topological twist assures that the respective RG defect descends to a defect between the topologically twisted IR and UV theories. We 
still refer to this defect as RG defect and to the topologically twisted theories as IR and UV theories. (Note that the notion of RG defects as defined in [1] does not require a topological twist. In fact, examples of RG defects are known in full CFTs [2]. We expect the ideas presented below to also be applicable in this more general context, albeit in a more intricate way.)

Fusion $^{1}$ of RG defects $R$ with their downward oriented versions $R^{\dagger}$ gives rise to the trivial identity defect in the IR theory, $R \otimes R^{\dagger} \cong I_{\mathrm{IR}}$, while fusion in the opposite order yields non-trivial defects $P=R^{\dagger} \otimes R$ in the UV theory:

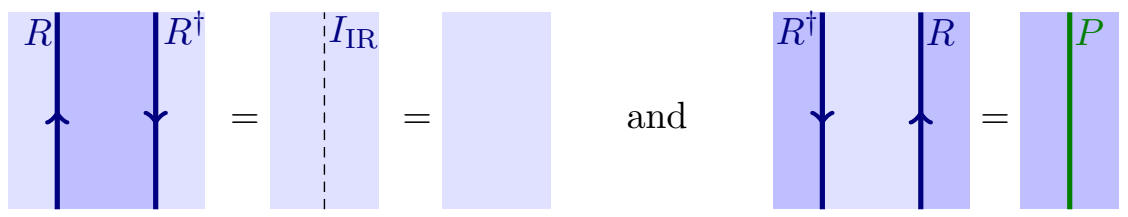

The first equation is a central property of RG defects, which ensures locality in the sense that islands of IR theories trivially connect:

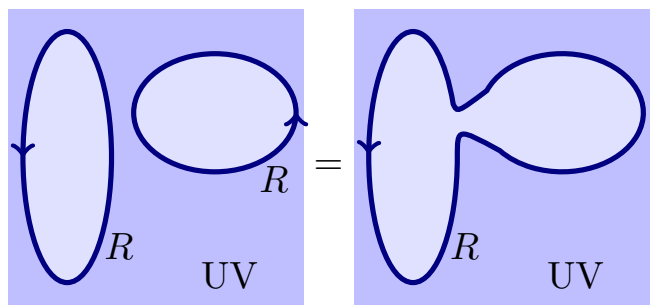

It also implies that the defects $P$ are projection defects, i.e. they are idempotent under fusion, $P \otimes P \cong P$. They project onto IR degrees of freedom in the UV theory.

Another consequence of (1.2) is that right $R$-loops evaluate to the identity:

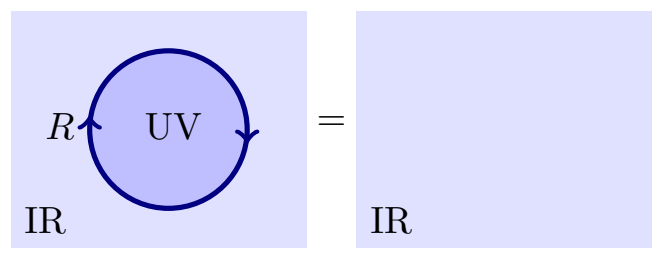

(Since the IR carries less information than the UV, the above loop-condition does not hold for left $R$-loops.) This can be used to express correlation functions of the IR theory in terms of UV correlators by the following trick familiar from the discussion of dualities and generalized orbifolds [3-6]: Because of equation (1.3), a given IR correlator is not changed upon insertion of right $R$ loops, cf. step I in the example (1.4) below. Since we are dealing with a topological quantum field theory, the UV islands created in this way can be expanded without changing the correlation function until they cover the entire space-time surface, see step II in (1.4). The result is a correlation function in the UV theory with a network of the projection defect $P$ inserted. For instance, a disk correlator in the IR with

\footnotetext{
${ }^{1}$ In a TQFT one can move parallel defects infinitely close together resulting in a new, fused defect. We will denote fusion of defects by ' $\otimes$ '.
} 

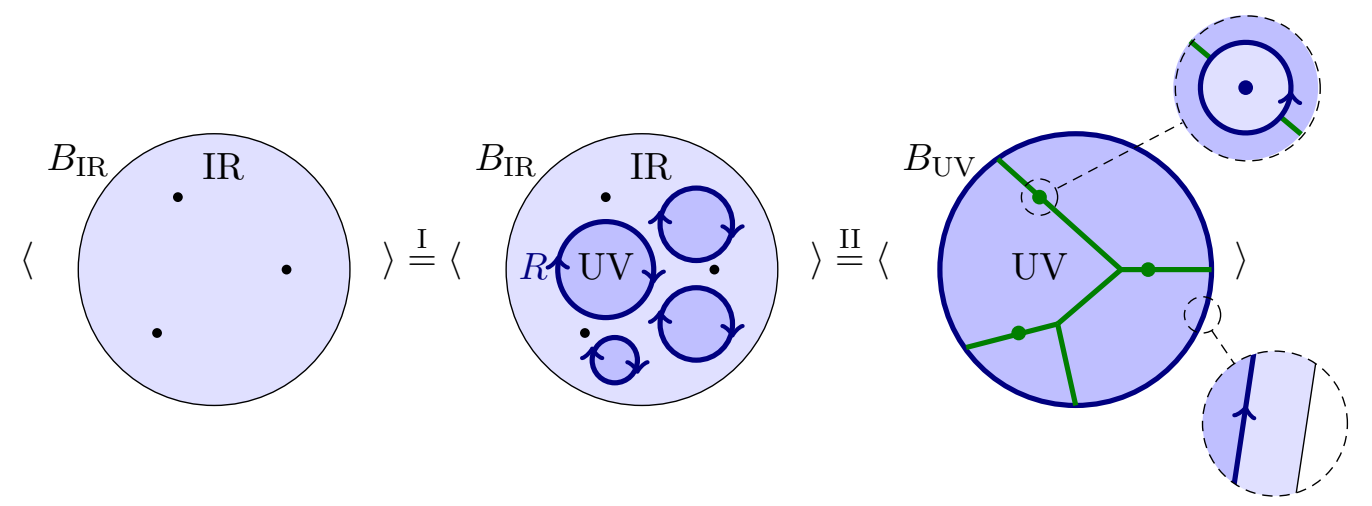

Of course, steps I and II involve many choices leading to representations of one and the same IR correlation function by possibly different $P$-networks in the UV. The latter can be related by sequences of local transformations generated by identities satisfied by the defects $P$ and their junctions.

Carrying out this procedure on the level of correlators immediately reveals how objects of the IR theory are represented in the UV. For instance, IR bulk fields appear as field insertions on the defect $P$. Right boundary conditions $B_{\mathrm{IR}}$ are mapped to boundary conditions $B_{\mathrm{UV}}=R^{\dagger} \otimes B_{\mathrm{IR}}$ in the UV. Similarly, IR defects $D_{\mathrm{IR}}$ are mapped to defects $D_{\mathrm{UV}}=R^{\dagger} \otimes D_{\mathrm{IR}} \otimes R$ in the UV. This in particular applies to the defects associated to symmetries of the IR theory. These symmetry defects encode the action of the symmetry group on all objects of the theory, and they fuse according to the multiplication in the symmetry group. Lifting IR symmetry defects to the UV one obtains UV defects, whose fusion is still governed by the IR symmetry group. This yields a realization of the IR symmetry group in the UV, which however is not an honest representation. After all, the identity defect in the IR corresponding to the neutral element in the IR symmetry group is lifted to the projection defect $P$ in the UV. Thus, the lifted symmetries are only invertible on the IR degrees of freedom.

In fact, given the projection defect $P$, the objects in the UV theory representing IR objects can be characterized completely within the UV theory without any reference to $R$ : IR bulk fields are represented by defect fields on $P$, right IR boundary conditions are represented by right UV boundary conditions $B_{\mathrm{UV}}$ which are invariant under fusion with $P, P \otimes B_{\mathrm{UV}} \cong B_{\mathrm{UV}}$. IR defects are represented by defects $D_{\mathrm{UV}}$ in the UV, which are invariant under fusion with $P$ from both sides, $D_{\mathrm{UV}} \otimes P \cong D_{\mathrm{UV}} \cong P \otimes D_{\mathrm{UV}}$, etc. Given the respective projection defect $P$, one can therefore completely describe the IR theory in the framework of the UV theory.

Through perturbations by different relevant operators, a UV theory might permit many different RG flows leading to possibly different IR theories at various engery scales. All of these theories with all their symmetries etc. can be described by projection defects in one and the same UV theory. This applies in particular if the theory is asympotically free in the UV, in which case all possible IR theories can be realized by means of projection defects in a free theory. 
Remarkably, the description of IR correlators in terms of UV correlators containing networks of the projection defect $P=R^{\dagger} \otimes R$ provides a radically new view on bulk perturbations: instead of perturbing the theory on the entire space-time, we can restrict the perturbation on a network of thin strips. These strips can even be made infinitely thin, effectively reducing the bulk perturbation to a (one-dimensional) perturbation of the identity defect in the UV theory. RG flow leaves the bulk theory at the UV, but drives the identity defect to some projection defect $P$ in the IR.

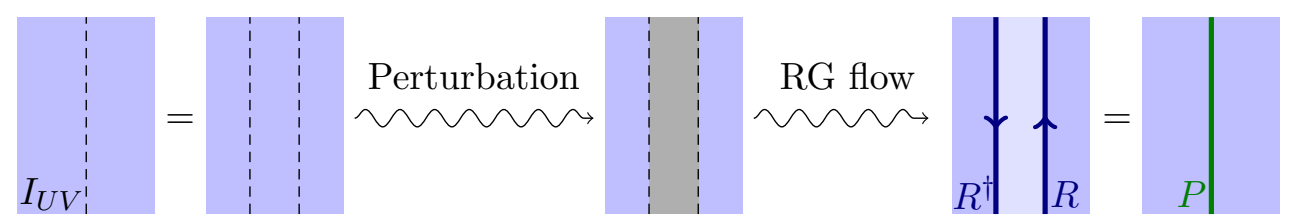

Concretely, one obtains the correlation function of the IR theory from the one at UV by first inserting an (invisible) network of the identity defect, which in particular passes through all bulk insertions and runs parallel to every boundary and also on both sides of any defect. The IR correlation function can then be obtained by a defect RG flow on this network.

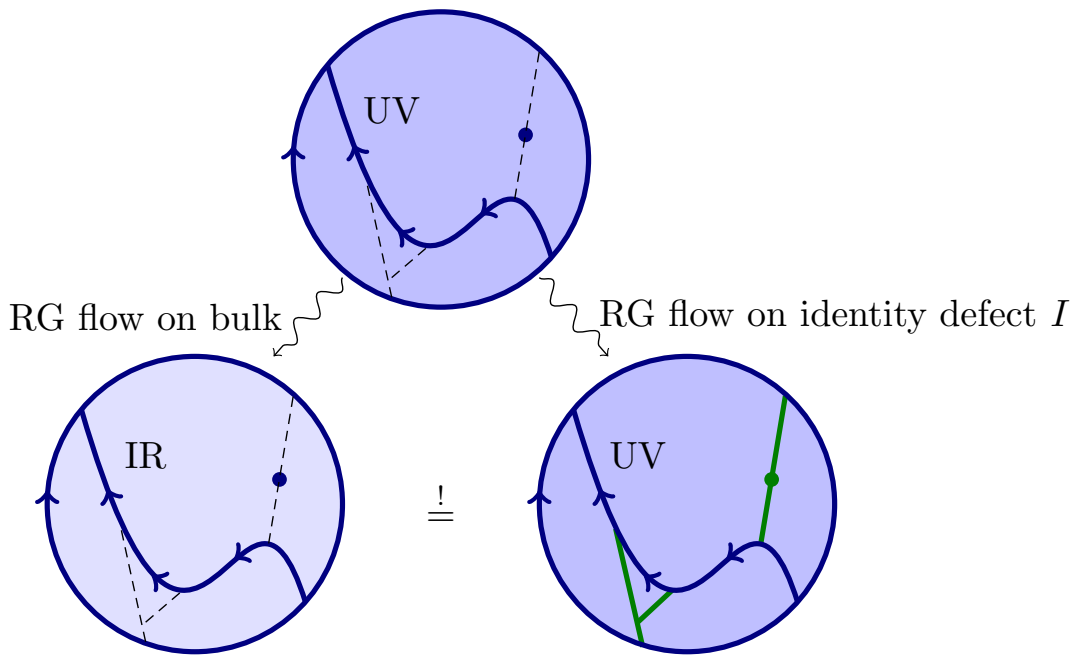

Under the flow UV boundary conditions and defects flow to their respective fusion with $P$. In this way, bulk RG flows can be entirely studied in the fixed UV bulk theory by means of perturbations of the UV identity defect.

The fact that one can describe IR theories in the UV without reference to the RG defects $R$ by using the respective projection defects $P=R^{\dagger} \otimes R$ suggests applying this procedure to general projection defects $P$, which do not a priori arise from RG flows. In this way, new ' $P$-projected' theories can be constructed from any projection defect $P$ in a given TQFT.

Interestingly, it turns out that any projection defect factorizes as $P=R^{\dagger} \otimes R$ for some defect $R$ separating the $P$-projected theory from the original one. What is more, the defect $R$ satisfies the locality property of RG defects, i.e. the left equation of (1.2). Hence, in fact all projection defects factorize into RG type defects. 
The construction described above is in fact closely related to the generalized orbifold procedure $[4,5,7,8]$. It differs from it in that we drop the technical assumption that left and right adjoints of defects agree $D^{\dagger} \cong{ }^{\dagger} D$, which is in particular not satisfied in the examples we present in this paper: the flows between orbifolds of Landau-Ginzburg models with a single chiral superfield studied in [1].

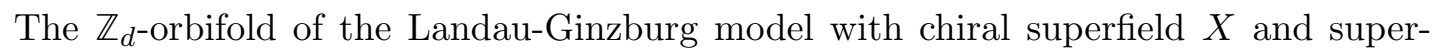
potential $W(X)=X^{d}$, which we denote by $\mathcal{M}_{d}$ admits RG flows to Landau-Ginzburg orbifolds $\mathcal{M}_{d^{\prime}}$ for all $d^{\prime}<d$. Applying the procedure described above to these flows yields a realization of all models $\mathcal{M}_{d^{\prime}}$ in terms of projection defects in $\mathcal{M}_{d}$ for $d^{\prime}<d$. In particular, taking $d \rightarrow \infty$ one obtains a representation of all models $\mathcal{M}_{d^{\prime}}$ in the theory of a free twisted chiral field.

The paper is organized as follows: In section 2 we briefly introduce defect lines in $2 \mathrm{~d}$ TQFTs, focussing on aspects which are important for our construction. The construction is then spelled out in section 3. Section 4 is devoted to a detailed discussion of the example of RG flows between Landau-Ginzburg orbifolds, in which the respective defects can be concretely described by means of matrix factorizations. We conclude with a discussion of open questions in section 5. Some more technical discussions and a brief outline of the generalized orbifold construction are relegated to various appendices.

\section{Defects in 2d TQFTs}

In this section, we set the stage by briefly introducing some aspects of defect lines in $2 \mathrm{~d}$ topological quantum field theories (TQFTs). We do this mainly to introduce notation. For more details on defect lines, see e.g. [9-11].

Defect lines are line operators, which (since they have codimension 1) can also separate different $2 \mathrm{~d}$ TQFTs on the same space-time surface. Locally, a neighborhood around a point on a defect $D: T \rightarrow T^{\prime}$ separating two TQFTs $T$ and $T^{\prime}$ can be depicted as ${ }^{2}$

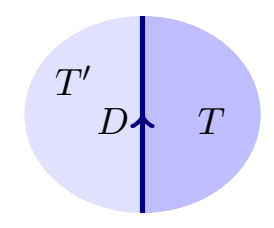

Defect lines carry local degrees of freedom, called defect fields, which can be inserted at points on defects. Defect fields can also separate different defects or glue together defects at junctions. We denote the space of defect changing fields between two defects $D, D^{\prime}: T \rightarrow T^{\prime}$ by $\operatorname{Hom}\left(D, D^{\prime}\right)$. Every defect carries the identity field $1_{D} \in \operatorname{Hom}(D, D)$.

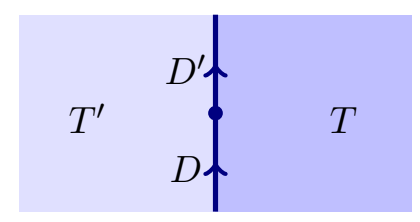

\footnotetext{
${ }^{2}$ Note that defect lines are oriented.
} 
In every $2 \mathrm{~d}$ TQFT $T$ there is a special invisible or identity defect $I_{T}$, whose insertion does not change correlation functions, and which can be connected to any other defect. The defect fields on this defect are just the bulk fields of the underlying 2d TQFT, $\operatorname{Hom}\left(I_{T}, I_{T}\right) \cong \mathcal{H}_{T}$.

Due to topological invariance, defects and field insertions can be moved on the spacetime surface without changing correlation functions, as long as field insertions or defects do not cross. This in particular implies an associative operator product on defect fields $\operatorname{Hom}\left(D, D^{\prime}\right) \otimes \operatorname{Hom}\left(D^{\prime}, D^{\prime \prime}\right) \rightarrow \operatorname{Hom}\left(D, D^{\prime \prime}\right)$.

Similarly, parallel defect lines can be brought close together, leading to the notion of defect fusion: When brought close together, defects $D^{\prime}: T^{\prime} \rightarrow T^{\prime \prime}$ and $D: T \rightarrow T^{\prime}$ fuse to the defect $D^{\prime} \otimes D: T \rightarrow T^{\prime \prime}$ :

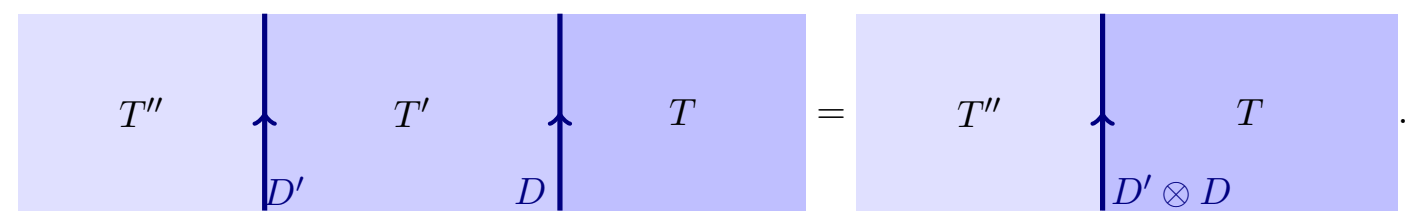

Topological invariance yields certain obvious compatibility relations between the operator product of defect fields and defect fusion, which we won't spell out here. We will however briefly mention one feature of topological defects, which will be of particular importance for our construction.

Due to topological invariance one can bend a defect (to the right or left) without changing correlators. This is described by the following two Zorro move identities (relations like this hold locally when inserted in any correlator):

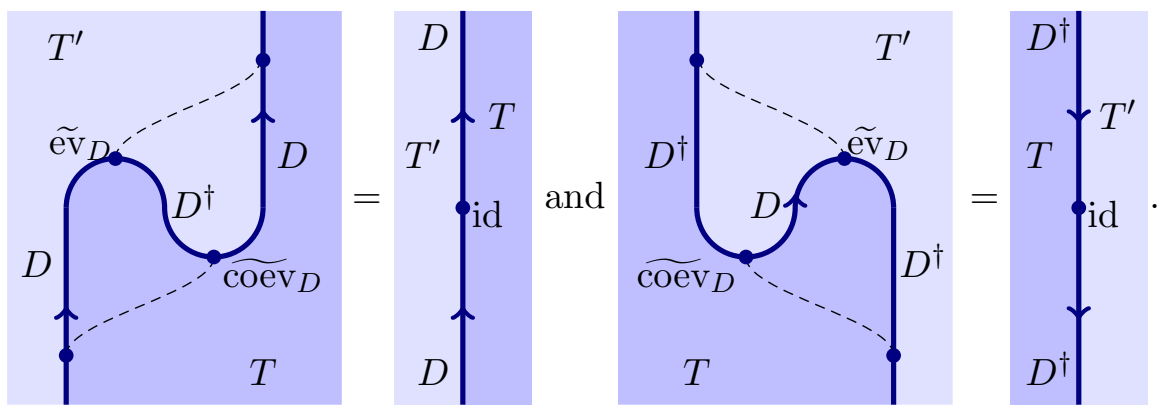

These diagrams involve additional structure: First of all, bending $D$ to the right results in a downwards oriented version $D^{\dagger}$ of $D$, its right-adjoint. Secondly, dotted lines depict the (invisible) identity defect $I$, which connects to the defects $D$ and $D^{\dagger}$ in defect (junction) fields

$$
\begin{array}{r}
\widetilde{\mathrm{ev}}_{D}: D \otimes D^{\dagger} \rightarrow I_{T^{\prime}} \\
\widetilde{\operatorname{coev}}_{D}: I_{T} \rightarrow D^{\dagger} \otimes D,
\end{array}
$$

called evaluation and coevaluation maps, respectively. Of course, one can equally well bend the defect $D$ to the left

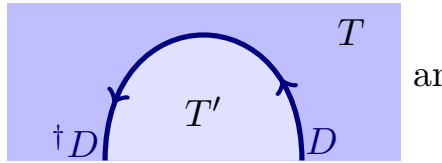

and

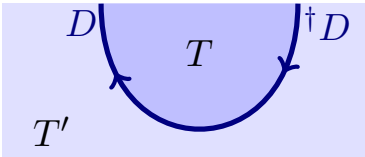


giving rise to the left-adjoint ${ }^{\dagger} D$ of $D$. Topological invariance implies an analogous Zorro move identity involving $D$ and ${ }^{\dagger} D$ and the respective (co-)evaluation maps

$$
\begin{gathered}
\operatorname{ev}_{D}:{ }^{\dagger} D \otimes D \rightarrow I_{T} \\
\operatorname{coev}_{D}: I_{T^{\prime}} \rightarrow D \otimes{ }^{\dagger} D .
\end{gathered}
$$

Of course for all defects $D,\left({ }^{\dagger} D\right)^{\dagger} \cong D \cong{ }^{\dagger}\left(D^{\dagger}\right)$. For more details on adjunctions of defects, see [12].

In the main text of this paper, defect loops will play an important role. The description of loops of a defect $D$ in this framework requires a morphism $\phi \in \operatorname{Hom}\left({ }^{\dagger} D, D^{\dagger}\right)$, by which such loops can be closed

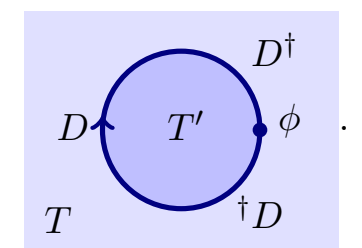

For many classes of $2 \mathrm{~d}$ TQFTs (such as non-orbifold LG-models with an even number of chiral fields), there is a canonical isomorphism $D^{\dagger} \cong{ }^{\dagger} D,{ }^{3}$ for any defect $D$, which can be used for this purpose. This is not true in general, however. So we cannot resort to these canonical maps. Instead, we will construct natural loop closing homomorphisms for the special class of defects which appear in our construction.

\section{RG-networks in 2d TQFTs}

\subsection{Projections from RG defects}

Starting point of our construction are RG defects as defined in [1]. These defects arise when $2 \mathrm{~d}$ field theories are perturbed by local operators only on part of the space-time surface. The RG flow drives the theory to the IR on the perturbation domain, while leaving it at the UV on the rest, thus creating a defect on the boundary of the perturbation domain separating the IR from the UV theory as in (1.1). This RG defect encodes all aspects of the relationship between UV and IR theories. ${ }^{4}$

Next, we pass to the context of $2 \mathrm{~d}$ topological quantum field theories via the topological twist. Indeed, we assume that the 2d QFT under consideration allows for a topological twist which moreover is compatible with the RG flow. ${ }^{5}$ Then the RG defects descend to topological defects between topologically twisted IR and UV theories, which we will still refer to as RG defects.

Arising from local perturbations, RG defects have rather special properties. Locality postulates that perturbations on two adjacent domains is nothing but the perturbation on

\footnotetext{
${ }^{3}$ or equivalently $D^{\dagger \dagger} \cong D$.

${ }^{4}$ For instance, in [1] RG-defects are used to describe how boundary conditions behave under perturbations of the bulk theory.

${ }^{5}$ Examples of such theories are $2 d N=(2,2)$ superconformal field theories perturbed by chiral or twisted chiral fields.
} 
the union of the domains. This implies that fusion of an RG defect $R$ with its opposite defect $R^{\dagger}$ in the UV theory yields the identity defect $I_{\mathrm{IR}}$ in the IR:

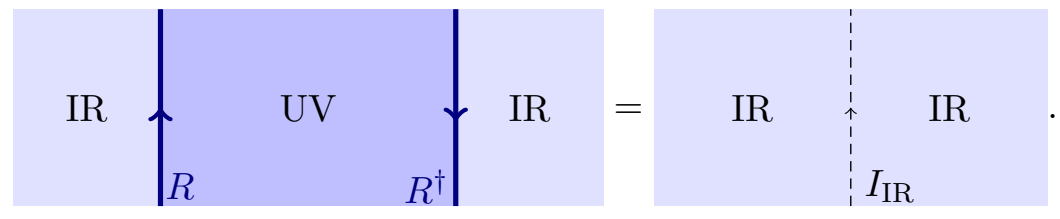

In other words, there is an isomorphism $R \otimes R^{\dagger} \cong I_{\mathrm{IR}}$ to the identity defect in the IR theory, which together with its inverse yields the following relations:
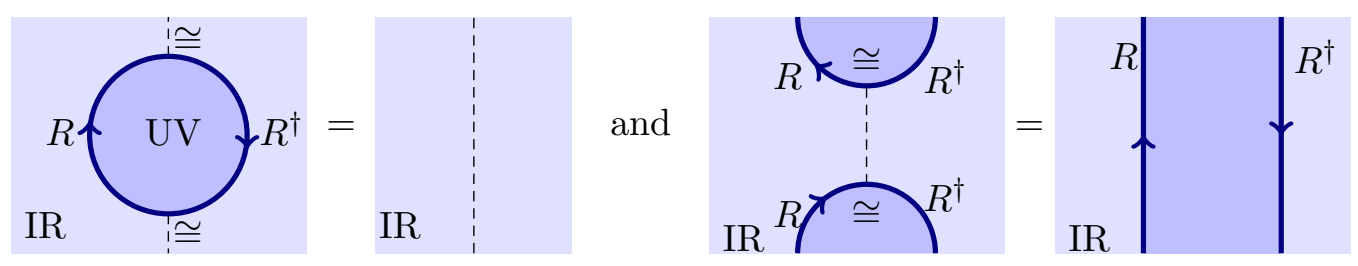

Since $I_{\mathrm{IR}}$ is self-adjoint, $R \otimes R^{\dagger} \cong I_{\mathrm{IR}}$ is equivalent to $R \otimes{ }^{\dagger} R \cong I_{\mathrm{IR}}$, and similar relations hold for ${ }^{\dagger} R$.

One important consequence of this is that one can close right $R$ loops in a way that makes them evaluate to the identity. (This is not true for left $R$ loops, i.e. those enclosing the IR theory, which are not invertible in the case of non-trivial RG flows.) The loopclosing morphism $\phi:{ }^{\dagger} R \rightarrow R^{\dagger}$ is obtained by moving the isomorphisms in the first relation in (3.1) along the defect to the right:
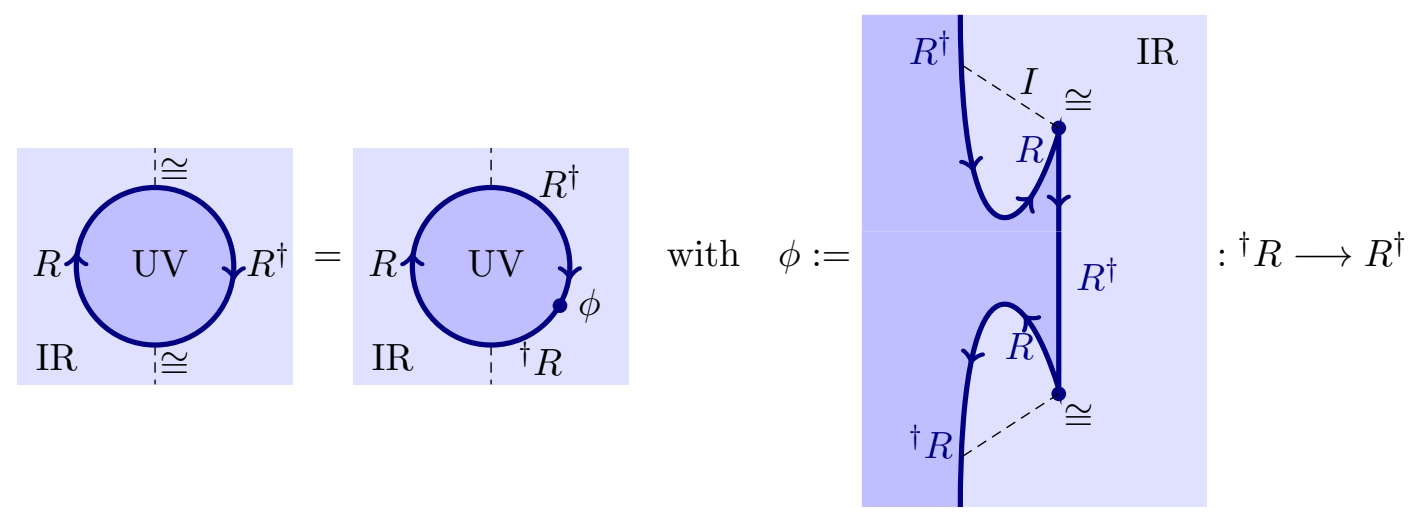

Utilizing that right $R$ loops evaluate to the identity it is possible to express correlation functions of the IR theory in terms of correlation functions in the UV by the following trick: Given a correlation function of the IR theory, one can insert right $R$ loops without changing it. Expanding these islands of UV theory until they cover the entire surface, the IR correlation function is transformed into a correlation function of the UV theory with a network of defects as in equation (1.4).

The network is built out of the defect $P:=R^{\dagger} \otimes R$ (in the following represented by green lines which we take as upwards oriented if an orientation is not specified) and 
its junctions
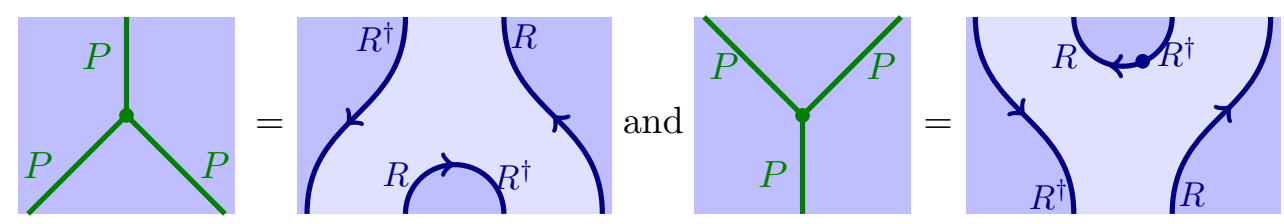

which we call multiplication and comultiplication, respectively.

The defect $P$ together with its junctions has some rather special features, which easily follow from the properties of $R$. In particular, $P \otimes P \cong P$, and the following relations hold:
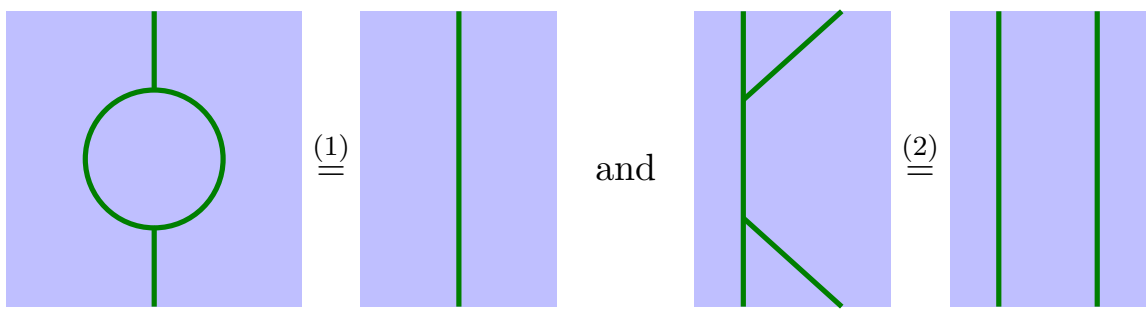

We call the first one loop-omission property (or separability) and the second one projection property. Beyond these, $P$ also obeys the following identities:

associativity:

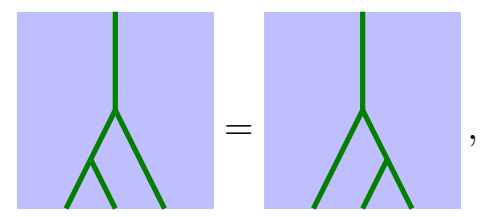

coassociativity:

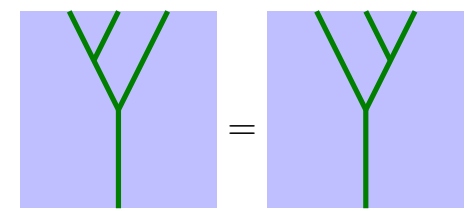

and the Frobenius identities:
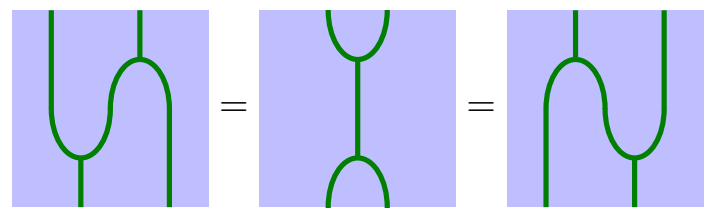

Moreover, $P$ comes with a unit

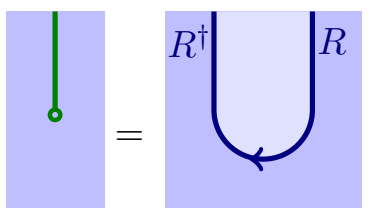

for which

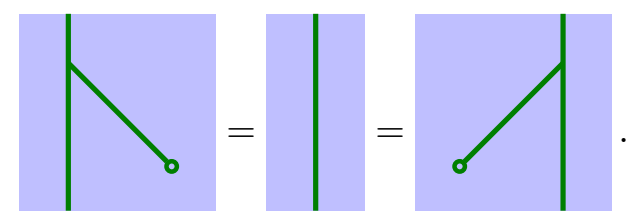

Indeed, instead of $P=R^{\dagger} \otimes R$ we could just as well have chosen $P^{\prime}={ }^{\dagger} P={ }^{\dagger} R \otimes R$ as building block of the network above. The latter defect equally satisfies the relations above with the only difference that instead of a unit, it has a counit

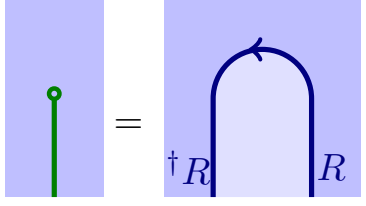

for which
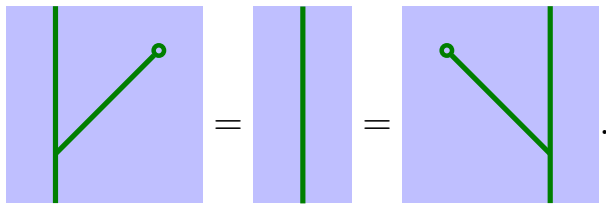

In summary, any correlation function of the IR theory can be written as a correlator in the UV with a $P$-network inserted. The correlation function is invariant under local changes 
of the $P$-network generated by loop-omission and projection properties, the associativity and coassociativity relations and the Frobenius identities. This in particular reflects the fact that the resulting correlation function does not depend on how exactly the UV islands are inserted into the IR correlators and how they are expanded.

\subsection{Representing the IR in the UV}

Having expressed the IR correlators in terms of UV correlators in the last section, we now discuss how the defining structures of IR correlators such as bulk fields, boundaries, defects and symmetries are represented in the UV theory. The results can be summarized as follows: If one characterizes the respective IR object by its relation to the IR identity defect $I_{\mathrm{IR}}$, then its UV realization is obtained by replacing the IR identity defect by the defect $P$ of the UV theory, cf. table 1 . For simplicity we will restrict the discussion to the case of unital projection defect $P=R^{\dagger} \otimes R$. The results are the same for the counital case, and the argument is similar.

IR bulk fields. Let us first discuss bulk fields of the IR theory. Upon expanding the UV islands in the IR, bulk fields become defect fields on $P$, i.e. elements in $\operatorname{Hom}(P, P)$ (represented in diagrams by dots on defects). Due to topological invariance, they have to be compatible with the multiplication on $P$. Namely,

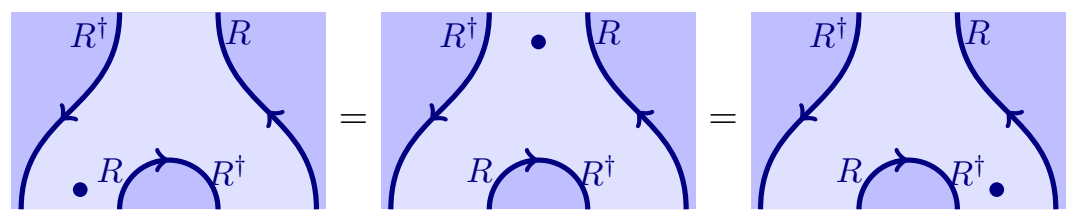

implying

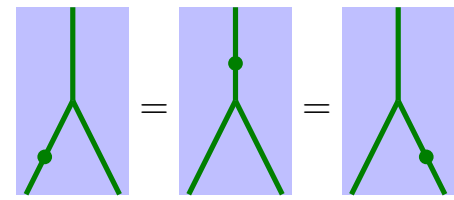

Considering the algebra $P$ as $P$-bimodule, the IR bulk fields become $P$-bimodule morphisms of $P$ in the UV. By the same argument these morphisms also respect the $P$-comodule structure on $P$ :

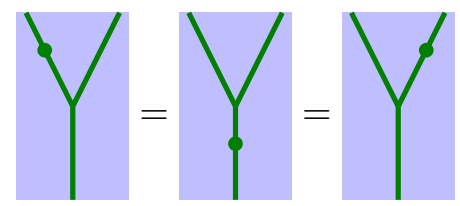

Now, not only are IR bulk fields lifted to $P$-bimodule morphisms of $P$ in the UV, the Hilbert space of bulk fields of the IR theory is in fact isomorphic to the space of $P$-bimodule morphisms of $P$. More precisely, the map

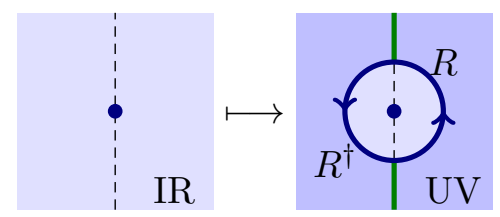




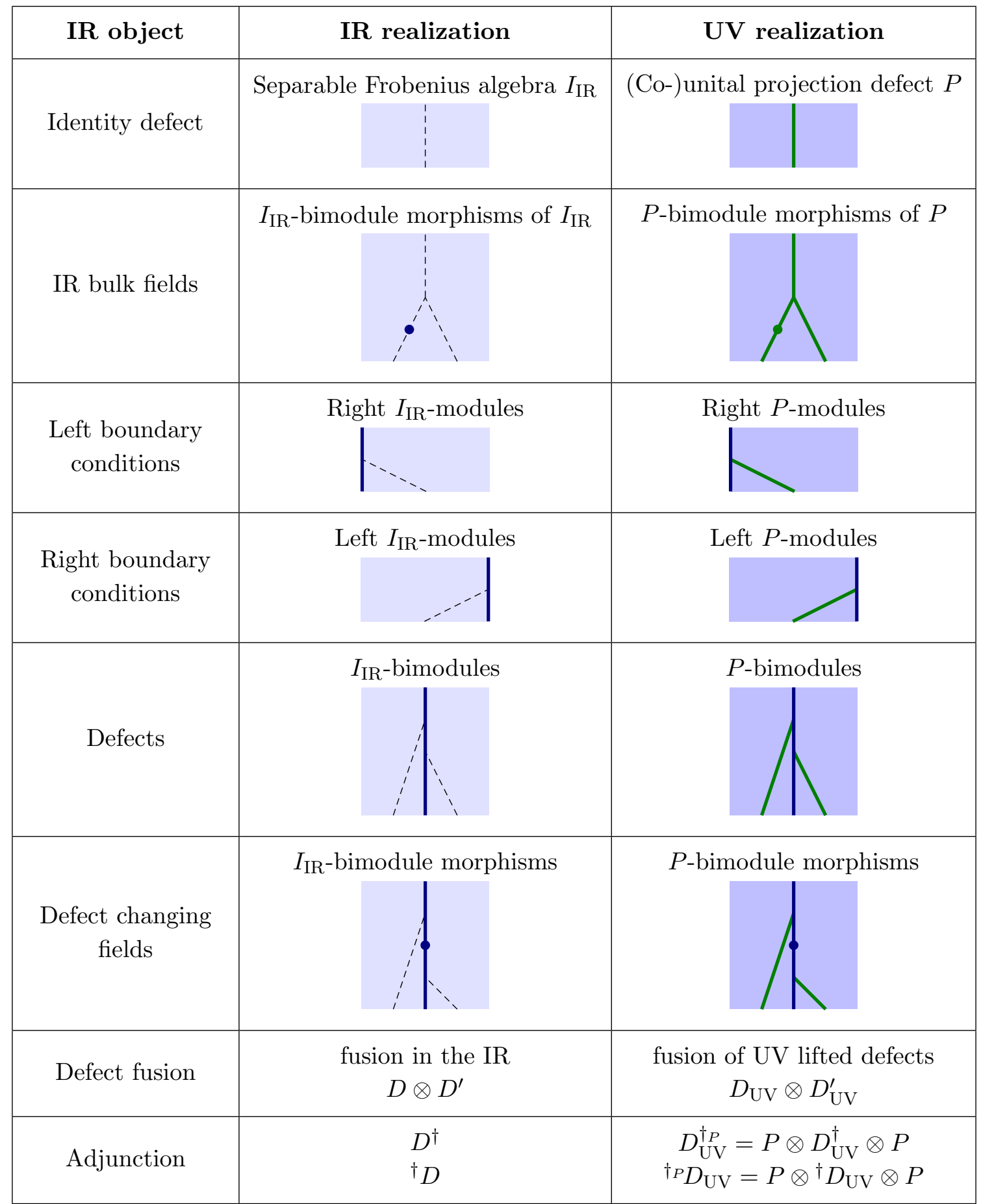

Table 1. Dictionary of IR structures lifted into the UV. 
sending IR bulk fields to $P$-bimodule morphisms of $P$ is an isomorphism. This is spelled out in appendix A.1.

In fact, due to the special properties of $P$, all morphisms of $P$, i.e. all defect fields on $P$ are automatically $P$-bimodule morphisms of $P$ and at the same time also $P$-bicomodule morphisms of $P$, see appendix A.2. Thus, the IR bulk Hilbert space is isomorphic to the space of defect fields on $P$.

IR boundary conditions and defects. Next, let us discuss left IR boundary conditions. Upon inserting and expanding UV islands in the IR theory, a left IR boundary condition $B_{\mathrm{IR}}$ is lifted to the UV boundary condition $B_{\mathrm{UV}}:=B_{\mathrm{IR}} \otimes R$. The latter comes equipped with a map

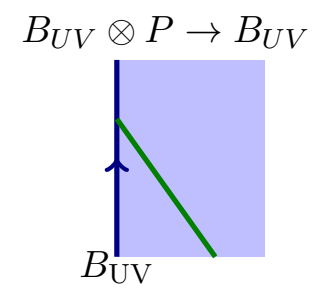

arising from

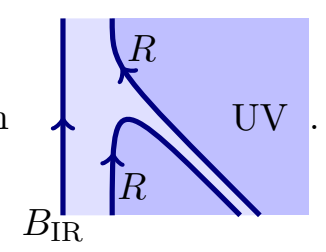

It satisfies the identities
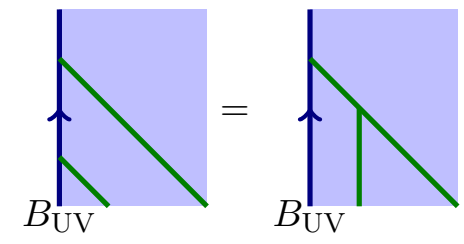

and

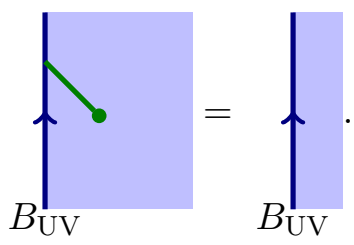

In other words, $B_{\mathrm{UV}}$ is a right $P$-module. In fact, the unit of $P$ induces a $P$-comodule structure on any $P$-module, hence also on $B_{\mathrm{UV}}$ :

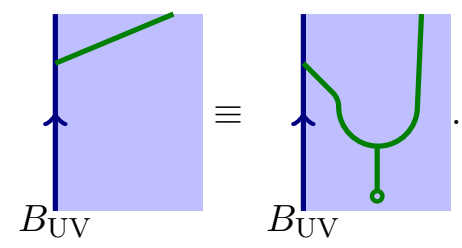

Therefore, left IR boundary conditions lift to right $P$-modules in the UV, which automatically are also $P$-comodules.

Conversely, all right $P$-modules arise in this way from IR boundary conditions. To see this, note that due to the special properties of the defect $P$, a left UV boundary condition $B$ is a right $P$-module iff $B \cong B \otimes P$ as shown in appendix A.3. Hence, given a right $P$-module $B$, the IR boundary condition $B_{\mathrm{IR}}=B \otimes R^{\dagger}$ satisfies $B_{\mathrm{IR}} \otimes R=B \otimes R^{\dagger} \otimes R=B \otimes P \cong B$. Thus, left IR boundary conditions are in one-to-one correspondence with right $P$-modules in the UV. ${ }^{6}$

\footnotetext{
${ }^{6}$ Indeed, this also holds if one chooses to construct the network using $P^{\prime}={ }^{\dagger} R \otimes R$ instead of $P=R^{\dagger} \otimes R$. In that case however $B_{U V}=B_{I R} \otimes{ }^{\dagger} R$ inherits a natural $P^{\prime}$-comodule structure, which by means of the counit on $P^{\prime}$ also induces a $P^{\prime}$-module structure on $B_{U V}$.
} 
Analogously one finds that right IR boundary conditions $B_{\mathrm{IR}}$ lift to left $P$-modules $B_{\mathrm{UV}}=R^{\dagger} \otimes B_{\mathrm{IR}}$ in the UV, and defects $D_{\mathrm{IR}}$ of the IR theory lift to $P$-bimodules $D_{U V}=$ $R^{\dagger} \otimes D_{\mathrm{IR}} \otimes R$. Importantly, $P$ itself is the UV lift of the IR identity defect:

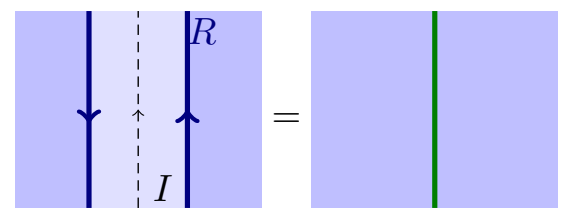

A straight-forward generalization of the discussion of IR bulk fields shows that IR defect fields are lifted to bimodule morphisms of the respective UV lifted defects, which again due to the special properties of $P$ are nothing but the defect fields of the UV lifts.

Fusion of IR defects. Because of $R \otimes R^{\dagger} \cong I_{\mathrm{IR}}$, the lift of fused IR defects is the fusion of the lifted defects:

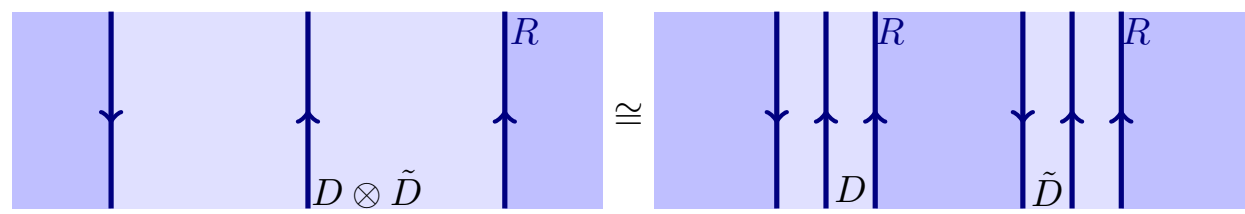

This is a rather special property closely tied to the projection property of $P$.

Adjunction of IR defects. While fusion of defects in the IR lifts to fusion in the UV, adjunction is not compatible with the lift from IR to UV. If for instance, an IR defect $D_{\text {IR }}$ is lifted to a defect $D_{\mathrm{UV}}=R^{\dagger} \otimes D_{\mathrm{IR}} \otimes R$ in the IR, then the right adjoint of the latter in the UV theory is given by $D_{\mathrm{UV}}^{\dagger}=R^{\dagger} \otimes D_{\mathrm{IR}}^{\dagger} \otimes R^{\dagger \dagger}$, which in general does not coincide with the lift $R^{\dagger} \otimes D_{\mathrm{IR}}^{\dagger} \otimes R$ of the right adjoint of $D_{\mathrm{IR}}$ to the UV theory. However, the two are related: Selfadjointness of the IR identity defect yields $R^{\dagger \dagger} \otimes R^{\dagger} \cong I_{\mathrm{IR}}$, and hence the UV lift of the adjoint can be expressed as $R^{\dagger} \otimes D_{\mathrm{IR}}^{\dagger} \otimes R \cong D_{\mathrm{UV}}^{\dagger} \otimes P \cong P \otimes D_{\mathrm{UV}}^{\dagger} \otimes P$, leading to the notion of IR adjunction in the UV theory, which we denote by

$$
D_{\mathrm{UV}}^{\dagger P}:=P \otimes D_{\mathrm{UV}}^{\dagger} \otimes P
$$

Similarly, the UV lift of a left adjoint defect is given by ${ }^{7}$

$$
{ }^{\dagger} D_{\mathrm{UV}}=P \otimes{ }^{\dagger} D_{\mathrm{UV}} \otimes P .
$$

These formulas are very natural. After all, the defining relation of adjoints are the Zorro move identities (2.1), which involve the identity defect. Lifting these identities from the

\footnotetext{
${ }^{7}$ The same formulas for left and right IR adjunction hold if one chooses to construct the network using the counital $P^{\prime}={ }^{\dagger} R \otimes R$ instead of $P=R^{\dagger} \otimes R$.
} 
IR theory to the UV replaces the identity defect with the defect $P$ :

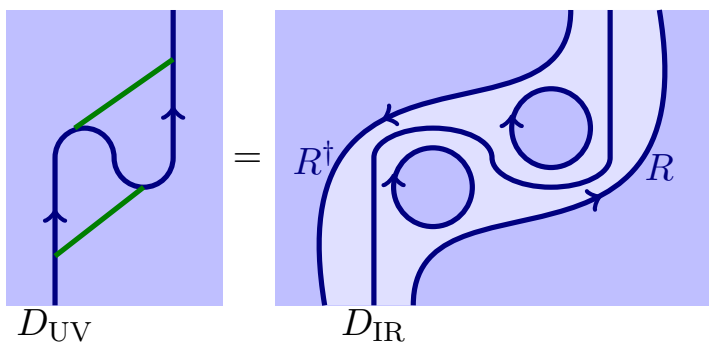

For instance, lifting the IR Zorro move identities for the right adjoint to the UV results in the relations
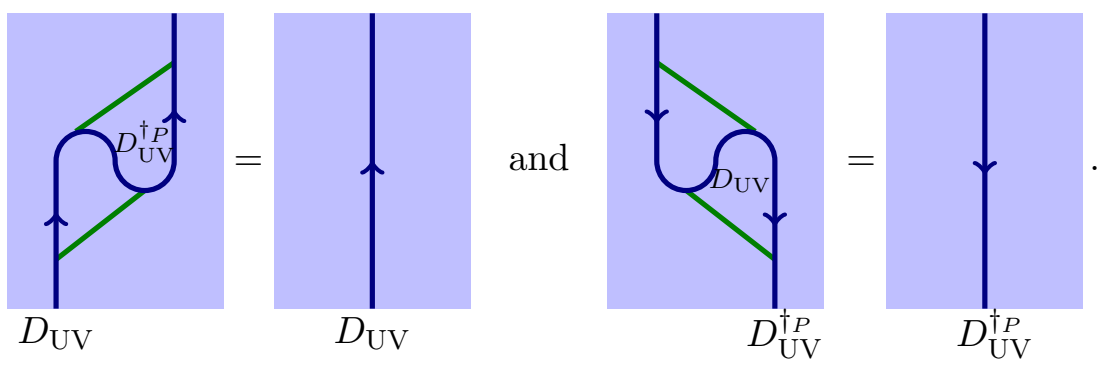

It is easy to see that fusing the UV adjoint from both sides with $P$ yields a defect which satisfies the $P$-Zorro move identities, cf. appendix A.4.

A special case is $P$ itself: Since it is the UV lift of the IR identity defect, which is selfadjoint, $P$ is selfadjoint with respect to $P$-adjunction: $P \cong P^{\dagger} P=P \otimes P^{\dagger} \otimes P$.

IR symmetries. Also symmetries of the IR theory can be easily described in the UV. As noted in [3] (see also [13]), symmetries of $2 \mathrm{~d}$ field theories can be described by symmetry defects ${ }_{g} I$ which describe the action of an element $g$ of the symmetry group on any object in field theory. The symmetry defects fuse according to the multiplication in the symmetry group: ${ }_{g} I \otimes_{h} I={ }_{g} \cdot h I(g \cdot h$ denotes the product in the symmetry group). Now, IR symmetry defects lift to the UV as any other defect: ${ }_{g} I \mapsto{ }_{g} I_{\mathrm{UV}}=R^{\dagger} \otimes_{g} I \otimes R$. Since IR fusion lifts to UV fusion, the fusion of the lifted symmetry defects still respects the multiplication in the symmetry group, ${ }_{g} I_{\mathrm{UV}} \otimes{ }_{h} I_{\mathrm{UV}}={ }_{g \cdot h} I_{\mathrm{UV}}$. In that sense, the IR symmetry group is already present in the UV theory. However it is not realized as a symmetry group in the UV, since the lift of the IR identity defect, which is the symmetry defect associated to the neutral element of the symmetry group, does not lift to the identity defect, but rather to $P$. So the lifted symmetry defects are in general not invertible defects in the UV, but instead satisfy ${ }_{g} I_{\mathrm{UV}} \otimes g_{g^{-1}} I_{\mathrm{UV}}=P$.

IR projectors and subsequent flows. Projection defects $P_{2}=\left(R_{2}\right)^{\dagger} \otimes R_{2}$ in the IR theory associated to some RG flow from the IR theory to some theory $\mathrm{IR}_{2}$ can also be lifted to the UV. The corresponding defects in the UV theory are given by

$$
\widetilde{P}=R^{\dagger} \otimes P_{2} \otimes R=R^{\dagger} \otimes R_{2}^{\dagger} \otimes R_{2} \otimes R=\left(R_{2} \otimes R\right)^{\dagger} \otimes\left(R_{2} \otimes R\right) .
$$

These are precisely the projection defects built out of the RG defect $R_{2} \otimes R$ associated to the concatenation of RG flows from the UV via IR to theory $\mathrm{IR}_{2}$. 
IR correlation functions. Having described how to realize IR objects inside the UV theory, it is straight-forward to represent IR correlators in the UV theory: First, prepare the IR correlator by placing identity defects through all field insertions, in particular bulk fields and at defect cusps. Then replace all IR objects by the respective UV objects as described above. Importantly, this includes the IR identity defect, which has to be replaced by the UV projection defect $P$. The resulting UV correlator coincides with the original IR correlator.

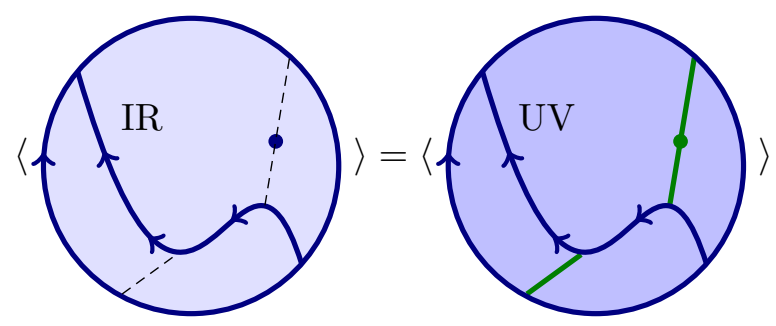

\subsection{Bulk RG flow as defect flow}

The previous discussion suggests a radically new view on bulk RG flow. Namely, that bulk perturbations of a $2 \mathrm{~d}$ theory can be understood as a perturbation of a defect network in the fixed UV bulk theory. More precisely, insertion and expansion of UV islands in the perturbed theory confines the perturbation on ever smaller domains, which eventually become one-dimensional. Hence, perturbed correlation functions are nothing but UV correlation functions with networks of perturbed identity defects inserted. RG flow then does not change the bulk UV theory, but only drives the identity defect in the UV to some projection defect $P$, cf. (1.5).

The two-dimensional RG flow in the bulk can hence be reduced to a one-dimensional RG flow on the identity defect $I_{\mathrm{UV}}$. Such defect flows are of course much easier to handle, because the underlying bulk theory does not change. For instance, UV bulk fields $\left(I_{\mathrm{UV}^{-}}\right.$ endomorphisms $)$ and boundaries ( $I_{\mathrm{UV}}$-modules) flow to bulk fields and boundaries in the UV theory, which are compatible with $P$, i.e. to $P$-bimodule morphisms of $P$ and $P$ modules, respectively.

Thus, if one can get a handle on perturbations of the identity defect in a given TQFT, the structures (bulk space, boundaries, correlators, etc.) associated to the corresponding perturbed bulk theory can be easily extracted.

\subsection{IR theories from projections}

In the previous discussion, we represented correlation functions of a perturbed $2 \mathrm{~d}$ TQFT as correlation functions of the unperturbed UV theory with a defect network inserted. While the starting point of the construction were RG defects $R$, the correlation functions of the perturbed theory only depended on the projection defect $P=R^{\dagger} \otimes R$. This suggests applying this method to arbitrary unital or counital projection defects $P$, which have the same properties as the defects associated to RG flows discussed in section 3.1: The 
projection property, $P \otimes P \cong P$ means that there are two junctions ${ }^{8}$

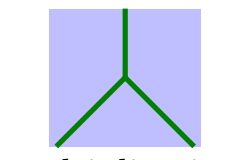

multiplication and

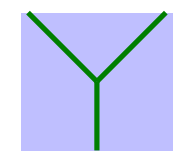

comultiplication

satisfying the loop-omission (separability) and projection properties:

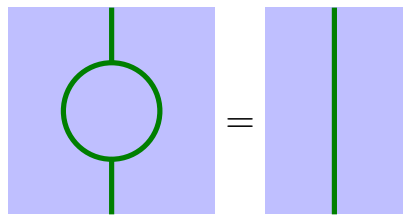

and

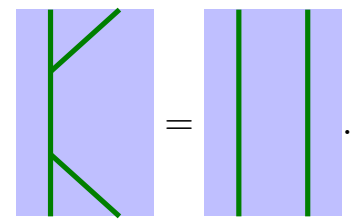

The junctions turn $P$ into an algebra as well as a coalgebra. We require $P$ to either have ${ }^{9}$

a unit
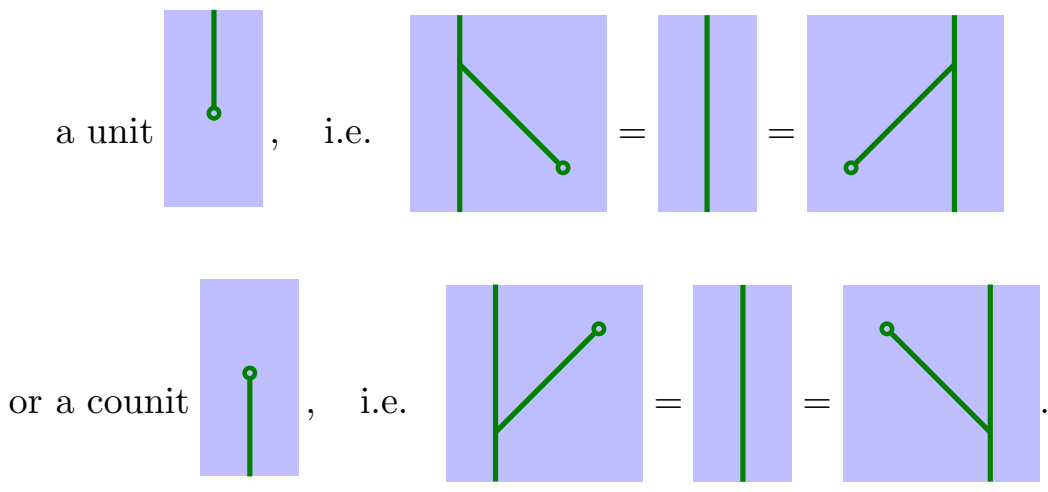

As is shown in appendix A.6, the existence of a unit for a projection defect implies coassociativity, while the existence of a counit implies associativity. In fact, for projection defects, associativity, coassociativity and the Frobenius identities

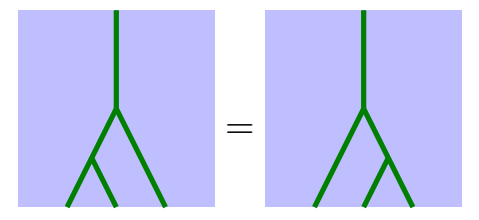

and
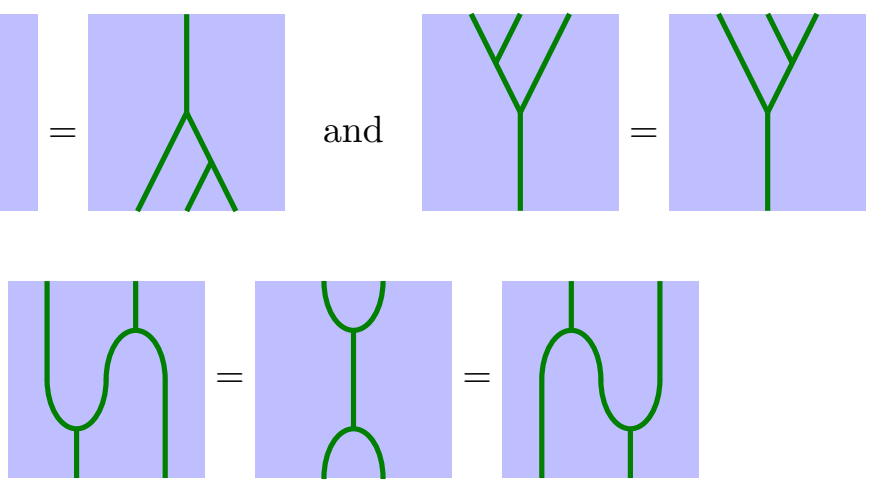

are all equivalent to one another, cf. appendix A.6. Thus, unital or counital projection defects satsify all of them.

${ }^{8}$ As before, $P$ is depicted in green, oriented from bottom to top.

${ }^{9}$ The special case in which $P$ has a unit as well as a counit is discussed in appendix A.5. 
As in the context of RG defects discussed in section 3.2, replacing the identity defect $I$ in a $2 \mathrm{~d}$ TQFT by an arbitrary projection defect $P$, and inserting $P$ networks into the correlation functions one obtains correlation functions of a new, $P$-projected $2 \mathrm{~d}$ TQFT. The relation between the projected and unprojected theories is exactly the same as the relation between IR and UV theories discussed in section 3.2.

While apriori, projection defects $P$ do not arise from a bulk perturbations, we will show in the next section that in fact they always factorize into RG type defects.

\subsection{Factorization of projection defects}

We now come full circle by showing that any (unital or counital) projection defect $P$ factorizes as

$$
\begin{array}{ll}
P=R^{\dagger} \otimes R & \text { in case } P \text { is unital } \\
P={ }^{\dagger} R \otimes R & \text { in case } P \text { is counital }
\end{array}
$$

where $R$ is an RG type ${ }^{10}$ defect between the $P$-projected theory on one side and the original unprojected theory on the other. By analogy to the case of RG flows, we call the original, unprojected theory UV and the $P$-projected theory IR.

The basic idea is simple: as $P$ is a (co)algebra, it can be viewed as a left and/or right (co)module over itself. Thus, the defect $P$ can be regarded as a defect in the original (UV) theory (the defect $P$ itself), a defect in the $P$-projected (IR) theory (the identity defect), or a defect separating one of those from the other. To indicate which of the interpretations we are referring to, we denote the respective defects as $P_{\mathrm{UV} \mid \mathrm{UV}}, P_{\mathrm{IR} \mid \mathrm{IR}}$, $P_{\mathrm{IR} \mid \mathrm{UV}}$ or $P_{\mathrm{UV} \mid \mathrm{IR}}$, respectively. For instance, viewed as a left $P$-(co)module and a right $I_{\mathrm{UV}}$-(co)module $P$ represents the defect $P_{\mathrm{IR} \mid \mathrm{UV}}$ between the $P$-projected (IR) theory and the original (UV) theory

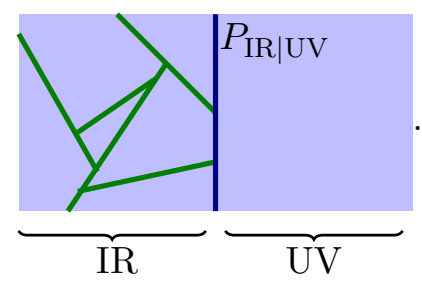

This defect plays the role of the RG defect $R$.

To show that it is indeed of RG type, we first need to determine its adjoints. We will restrict our discussion to the case that $P$ is unital. (There is an analogous argument for the case of counital $P$.) Since $P_{\mathrm{IR} \mid \mathrm{UV}}$ is a defect between IR and UV theory, the adjoints

\footnotetext{
${ }^{10} R \otimes R^{\dagger}$ is isomorphic to the identity defect.
} 
have to satisfy mixed Zorro identities:
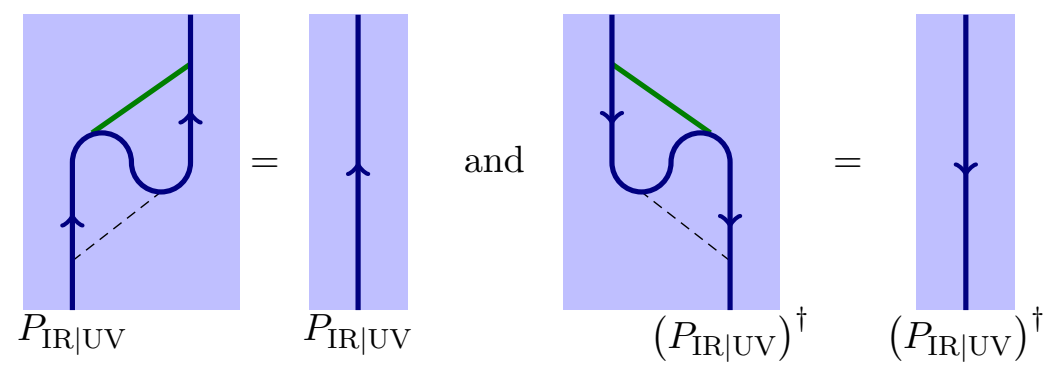

for the right adjoint and
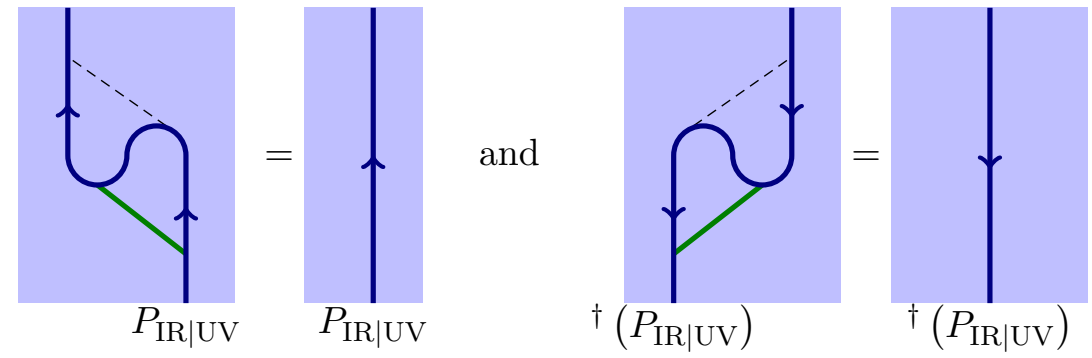

for the left adjoint. Here, the defect $P$ plays the role of the identity defect on the IR side of the defect. For unital $P$, comultiplication induces a coevaluation map $I_{\mathrm{UV}} \rightarrow P \rightarrow P \otimes P$, and, as is shown in appendix A.7

$$
\begin{aligned}
& \left(P_{\mathrm{IR} \mid \mathrm{UV}}\right)^{\dagger}=P_{\mathrm{UV} \mid \mathrm{IR}} \\
& { }^{\dagger}\left(P_{\mathrm{IR} \mid \mathrm{UV}}\right)=\left({ }^{\dagger} P\right)_{\mathrm{UV} \mid \mathrm{IR}} .
\end{aligned}
$$

( ${ }^{\dagger} P$ denotes the left adjoint of $P$ in the UV theory.) Now, since fusion over the IR theory is the same as fusion in the UV, it follows from the projection property of $P$ that

$$
P_{\mathrm{UV} \mid \mathrm{UV}}=P \cong P \otimes P=P_{\mathrm{UV} \mid \mathrm{IR}} \otimes P_{\mathrm{IR} \mid \mathrm{UV}}=\left(P_{\mathrm{IR} \mid \mathrm{UV}}\right)^{\dagger} \otimes P_{\mathrm{IR} \mid \mathrm{UV}} .
$$

Moreover, the identity defect in the IR theory is represented by $P$ in the UV theory, and hence

$$
I_{\mathrm{IR}}=P_{\mathrm{IR} \mid \mathrm{IR}}=P \cong P \otimes P=P_{\mathrm{IR} \mid \mathrm{UV}} \otimes P_{\mathrm{UV} \mid \mathrm{IR}}=P_{\mathrm{IR} \mid \mathrm{UV}} \otimes\left(P_{\mathrm{IR} \mid \mathrm{UV}}\right)^{\dagger} .
$$

Thus, any unital projection defect $P$ factorizes as $P=R^{\dagger} \otimes R$, where $R=P_{\mathrm{IR} \mid \mathrm{UV}}$ has the property that $R \otimes R^{\dagger} \cong I_{\mathrm{IR}}$. Note that all the defects $R, R^{\dagger}$ and $I_{\mathrm{IR}}$ are represented by $P$ in the UV theory, and the isomorphism $R \otimes R^{\dagger} \rightarrow I_{\mathrm{IR}}$ and its inverse are just given by the multiplication and comultiplication of $P$, respectively. The loop-omission and projection property of $P$ then imply
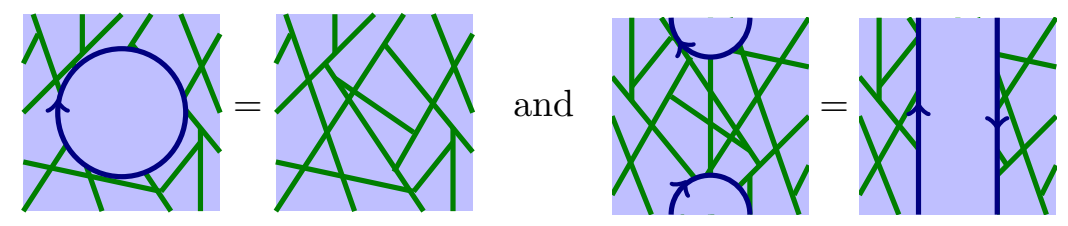
Similar considerations lead to an analogous factorization of counital projection defects $P$. The role of the RG defect is again played by $R=P_{\mathrm{IR} \mid \mathrm{UV}}$. But the adjoints differ from the unital case:

$$
\begin{aligned}
& \left(P_{\mathrm{IR} \mid \mathrm{UV}}\right)^{\dagger}=\left(P^{\dagger}\right)_{\mathrm{UV} \mid \mathrm{IR}} \\
& { }^{\dagger}\left(P_{\mathrm{IR} \mid \mathrm{UV}}\right)=P_{\mathrm{UV} \mid \mathrm{IR}},
\end{aligned}
$$

which leads to slightly different factorizations

$$
P_{\mathrm{UV} \mid \mathrm{UV}}=P \cong P \otimes P=P_{\mathrm{UV} \mid \mathrm{IR}} \otimes P_{\mathrm{IR} \mid \mathrm{UV}}={ }^{\dagger}\left(P_{\mathrm{IR} \mid \mathrm{UV}}\right) \otimes P_{\mathrm{IR} \mid \mathrm{UV}}
$$

and

$$
I_{\mathrm{IR}}=P_{\mathrm{IR} \mid \mathrm{IR}}=P \cong P \otimes P=P_{\mathrm{IR} \mid \mathrm{UV}} \otimes P_{\mathrm{UV} \mid \mathrm{IR}}=P_{\mathrm{IR} \mid \mathrm{UV}} \otimes^{\dagger}\left(P_{\mathrm{IR} \mid \mathrm{UV}}\right) .
$$

If $P$ comes with both, a unit and a counit, it is self-adjoint $\left(P^{\dagger} \cong P \cong{ }^{\dagger} P\right.$, see appendix A.5), and the left and right adjoint of the induced RG defect $R$ are isomorphic, $R^{\dagger} \cong{ }^{\dagger} R$.

\subsection{Relation to the generalized orbifold procedure}

The method described in section 3.5 above to construct a new 2d TQFT by replacing the identity defect by a projection defect $P$ is very close to and in fact inspired by the generalized orbifold procedure $[4,5,7,8,14,15]$ (see appendix B for a quick summary). In that procedure a new $2 \mathrm{~d}$ theory is defined from an original one by inserting networks of a defect $A$ into the correlation functions of the original theory. The difference to our construction is the requirements imposed on $A$.

In the generalized orbifold construction the defect $A$ has to be a separable Frobenius algebra, ${ }^{11}$ cf. appendix B. This condition is very similar to the properties of projection defects with two differences: On the one hand the defect $A$ does not have to satisfy the projection property, but is on the other hand required to have both, a unit and a counit, which we do not demand of projection defects. Moreover, it is often assumed in the generalized orbifold procedure that left and right adjoints of any defect $D$ are isomorphic, i.e. $D^{\dagger} \cong{ }^{\dagger} D$, so that further conditions such as pivotality and symmetry can be demanded (see e.g. [5]). We do not require such a condition, and in fact it is not met in our examples discussed in section 4 .

A projection defect $P$ has both a unit and counit if and only if left and right adjoints of the respective RG defects are isomorphic

$$
\left(P_{\mathrm{IR} \mid \mathrm{UV}}\right)^{\dagger} \cong P_{\mathrm{UV} \mid \mathrm{IR}} \cong{ }^{\dagger}\left(P_{\mathrm{IR} \mid \mathrm{UV}}\right)
$$

cf. appendix A.5. In that case $P$ is a separable Frobenius algebra, and the construction described in section 3.5 is a special case of the generalized orbifold construction. Indeed, the projection property of $P$ brings about interesting simplifications in the generalized orbifold construction, which we will spell out in the remainder of this section.

\footnotetext{
${ }^{11}$ a unital, counital, associative, coassociative algebra and coalgebra satisfying loop-omission and Frobenius properties.
} 
Let $A$ be a separable Frobenius algebra in a given $2 \mathrm{~d}$ theory. We will represent it by green line segments in diagrams. Defects in the generalized orbifold theory defined by $A$ are given by defects in the underlying $2 \mathrm{~d}$ theory, which are $A$-(bi)modules. Let $D$ and $\tilde{D}$ be two such (bi)modules. Their fusion in the generalized orbifold theory is given by their tensor product $D \otimes_{A} \tilde{D}$ over the algebra $A$, pictorially

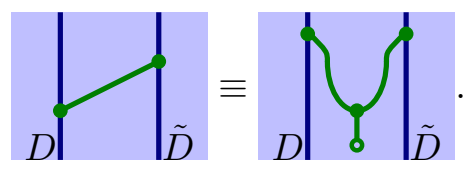

In general, it is different from the fusion $D \otimes \tilde{D}$ in the underlying unorbifolded theory.

Indeed, similarly to projection defects, also separable Frobenius algebras always factorize into defects between the orbifold and the underlying unorbifolded theory and their adjoints. Namely, considered as a left $A$ - and right $I$-module, ${ }^{12} A$ represents a defect $R$ between orbifolded and unorbifolded theory. Considered as right $A$ - and left $I$-module it represents the adjoint defect $R^{\dagger} \cong{ }^{\dagger} R$. Now, for any separable Frobenius algebra we have $A \otimes_{A} A \cong A$, or pictorially

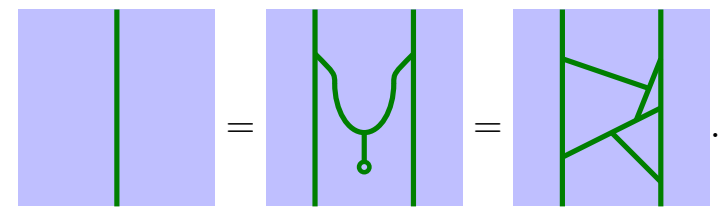

Hence, $A$ as a defect in the unorbifolded theory factorizes as $A \cong R^{\dagger} \otimes_{A} R$. However, for generic $A$ the defect $R$ is not of RG type, i.e. $R \otimes R^{\dagger}$ is not isomorphic to the identity defect in the orbifold theory. Hence, bubbles of a generalized orbifold theory inserted in the unorbifolded theory do not in general connect trivially:

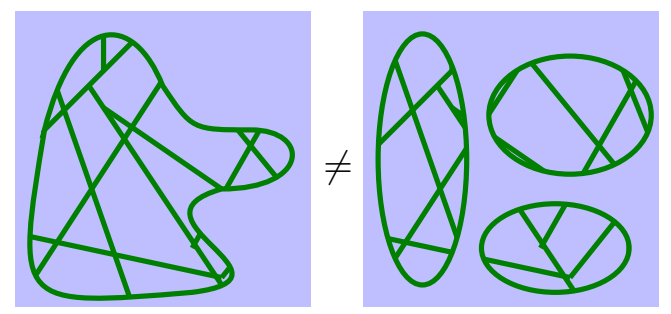

Instead, pushing two bubbles of the generalized orbifold against each other creates a nontrivial defect at the interface of the two bubbles. Thus, the generalized orbifold cannot be obtained by a local perturbation of the original theory. This is only true if $A$ additionally satisfies the projection property.

In that case, fusion in the generalized orbifold simplifies dramatically - it reduces to fusion in the unorbifolded theory. Namely, for a separable Frobenius algebra the projection property can be rephrased as

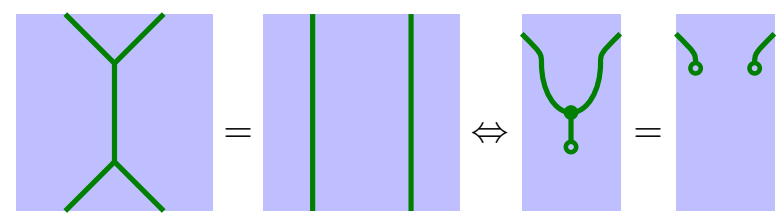

\footnotetext{
${ }^{12} I$ is the identity defect of the underlying unorbifolded theory.
} 
leading to the following simplification for defect fusion in the orbifold theory:

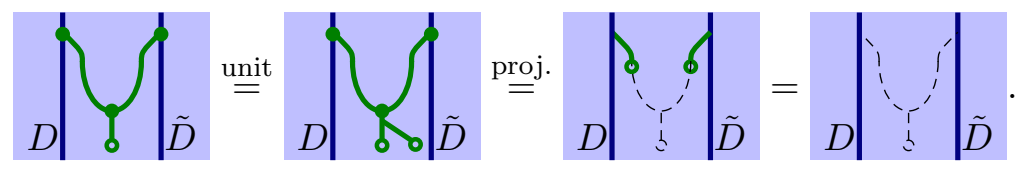

\section{RG-networks in Landau-Ginzburg orbifolds}

In this section we will apply our construction in the context of topologically twisted LandauGinzburg models. More precisely, we will consider Landau-Ginzburg models $\mathcal{M}_{d}$ with a single chiral superfield $X$ and superpotential $W(X)=X^{d}$. These models admit relevant perturbations generated by deformations of the superpotential by lower degree polynomials. For instance, the model $\mathcal{M}_{d}$ can be perturbed by adding a term $\lambda X^{d^{\prime}}$ to the superpotential. For $d^{\prime}<d$ this perturbation is relevant and the renormalization group flow drives the theory from the model $\mathcal{M}_{d}$ in the UV $(\lambda=0)$ to the model $\mathcal{M}_{d^{\prime}}(\lambda=\infty)$ in the IR. These perturbations are chiral and hence preserve A-type supersymmetry. The corresponding RG defects are therefore A-type defects. We prefer to work with B-type defects in LandauGinzburg models, because they are much better understood. For that reason, we will consider the mirror dual situation instead: RG flows between Landau-Ginzburg orbifolds $\mathcal{M}_{d} / \mathbb{Z}_{d}$ and $\mathcal{M}_{d^{\prime}} / \mathbb{Z}_{d^{\prime}}$ generated by twisted chiral perturbations. The respective RG defects have been constructed in [1].

We will start by giving a brief outline of the description of B-type defects in LandauGinzburg models by means of matrix factorizations. Then we will review the construction of the respective RG defects from [1]. Finally, we will use our construction to realize the IR theories by means of projection defects in the UV theories. In particular, we will show how to realize all the Landau-Ginzburg orbifolds $\mathcal{M}_{d} / \mathbb{Z}_{d}$ in the theory of a free twisted chiral field.

\subsection{B-type defects in Landau-Ginzburg models}

As put forward by Kontsevich, B-type defects in Landau-Ginzburg models can be described in terms of matrix factorizations [13, 16-19].

A matrix factorization of a polynomial $W \in S=\mathbb{C}\left[x_{1}, \ldots, x_{n}\right]$ consists of a $\mathbb{Z}_{2}$-graded free module $D=D_{0} \oplus D_{1}$ over the polynomial ring $S$, with an odd endomorphism $d_{D}$ : $D \rightarrow D$, which squares to $W$ times the identity map, i.e. $d_{D}^{2}=W \operatorname{id}_{D}$. One often unfolds matrix factorizations into 2-periodic complexes

$$
D: D_{1} \underset{\mathrm{d}_{D 0}}{\stackrel{\mathrm{d}_{D 1}}{\rightleftarrows}} D_{0}, \quad \mathrm{~d}_{D}=\left(\begin{array}{cc}
0 & \mathrm{~d}_{D 1} \\
\mathrm{~d}_{D 0} & 0
\end{array}\right) \text {. }
$$

These complexes are twisted by $W: d_{D 1} \circ d_{D 0}=W \operatorname{id}_{D_{0}}$ and $d_{D 0} \circ d_{D 1}=W \operatorname{id}_{D_{1}}$.

Now, topological Landau-Ginzburg models are completely specified by their chiral superfields $X_{1}, \ldots, X_{n}$ and their superpotential $W\left(X_{1}, \ldots, X_{n}\right)$. B-type defects $D$ between 
two LG models with superpotential $W\left(X_{1}, . ., X_{n}\right)$ and $V\left(Z_{1}, \ldots, Z_{m}\right)$

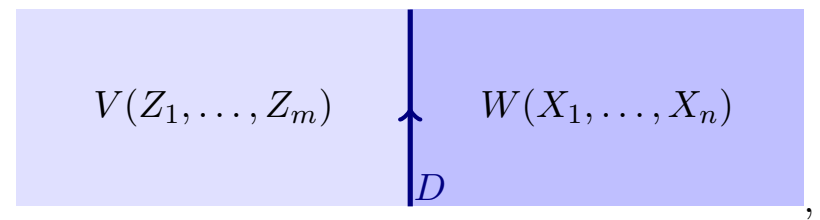

can be described by matrix factorizations of the difference $V-W$ of the respective superpotentials. By abuse of notation we also denote the matrix factorization by $D$ and write $D: W \rightarrow V$.

The space of defect-changing fields $\operatorname{Hom}\left(D, D^{\prime}\right)$ between two defects represented by matrix factorizations $D, D^{\prime}: W \rightarrow V$ is given by the homology of the induced $\mathbb{Z}_{2}$-graded complex on the space of homomorphisms $\operatorname{Hom}_{S}\left(D, D^{\prime}\right)$ of the respective $S$-modules. ${ }^{13}$ More precisely,

$$
\begin{aligned}
& \operatorname{Hom}\left(D, D^{\prime}\right)=H_{\mathrm{d}}^{*}\left(\operatorname{Hom}_{S}\left(D, D^{\prime}\right)\right), \\
& \quad \text { with differential } \mathrm{d} \phi=\mathrm{d}_{D^{\prime}} \circ \phi-(-1)^{\operatorname{deg}} \phi \circ \mathrm{d}_{D}, \quad \text { for } \quad \phi \in \operatorname{Hom}_{S}\left(D, D^{\prime}\right) .
\end{aligned}
$$

Here deg denotes the $\mathbb{Z}_{2}$-degree. The space of defect-changing fields is $\mathbb{Z}_{2}$-graded with even and odd elements corresponding to bosons and fermions, respectively. The operator product of defect-changing fields is just the composition of homomorphisms.

Defect fusion is described by the tensor product of matrix factorizations [13]. Namely, let $U \in \mathbb{C}\left[X_{1}, \ldots, X_{m}\right], V \in \mathbb{C}\left[Y_{1}, \ldots, Y_{n}\right], W \in \mathbb{C}\left[Z_{1}, \ldots, Z_{o}\right]$ and $D: W \rightarrow V$ and $D^{\prime}: V \rightarrow U$ be matrix factorizations of $V-W$ and $U-V$, respectively. Then the fused defect is given by the tensor product $D^{\prime} \otimes D$ of matrix factorizations. This is the matrix factorization built on the $\mathbb{Z}_{2}$-graded $\mathbb{C}\left[X_{1}, \ldots, X_{m}, Z_{1}, \ldots, Z_{o}\right]$-module $D^{\prime} \otimes_{\mathbb{C}\left[Y_{1}, \ldots, Y_{n}\right]}$ $D$ with homomorphism

$$
\mathrm{d}_{D^{\prime} \otimes D}=\mathrm{d}_{D^{\prime}} \otimes \mathrm{id}_{D}+\mathrm{id}_{D^{\prime}} \otimes \mathrm{d}_{D} .
$$

This differential is to be understood with Koszul signs, meaning that

$$
\left(\operatorname{id}_{D^{\prime}} \otimes \mathrm{d}_{D}\right)(\nu \otimes \omega)=(-1)^{\operatorname{deg}}(\nu) \otimes \mathrm{d}_{D}(\omega) .
$$

Since the factorized polynomials add upon taking the tensor product, this is indeed a matrix factorization of $(U-V)+(V-W)=U-W$, i.e. $D^{\prime} \otimes D: W \rightarrow U \cdot{ }^{14}$

Adjunctions of B-type defects in LG models have been studied in [12, 20] (see [21] for a nice review). Adjoints are given by

$$
D^{\dagger} \cong D^{\vee}[n], \quad{ }^{\dagger} D \cong D^{\vee}[m]
$$

where $D^{\vee}$ is the dual of a matrix factorization $D$, consisting of the dual modules $\left(D^{\vee}\right)_{i}=$ $\left(D_{i}\right)^{\vee}$, and the maps

$$
\mathrm{d}_{D^{\vee}}=\left(\begin{array}{cc}
0 & \mathrm{~d}_{D 0}^{\vee} \\
-\mathrm{d}_{D 1}^{\vee} & 0
\end{array}\right) .
$$

\footnotetext{
${ }^{13}$ Note that the Hom-complex is untwisted!

${ }^{14} \mathrm{~A}$ priori, tensor product matrix factorizations like this are of infinite rank. It can be shown however, that tensor products of finite-rank matrix factorizations are isomorphic to finite-rank matrix factorizations [13].
} 
Moreover, $(\cdot)[m]$ denotes the shift of $\mathbb{Z}_{2}$-degree by $m:(D[m])_{i}=D_{i+m}$ and $\mathrm{d}_{D[m] i}=$ $(-1)^{m} \mathrm{~d}_{D(i+m)}$.

Indeed, boundary conditions are a special case of defects, namely those with a trivial theory on one side. The trivial LG theory is of course the theory with no chiral fields and zero superpotential. Right (left) B-type boundary conditions of a Landau-Ginzburg theory with superpotential $W$ can therefore be described by matrix factorizations of $W(-W)$.

B-type defects in Landau-Ginzburg orbifolds. The description of B-type defects in Landau-Ginzburg models by means of matrix factorizations extends in a straight-forward manner to the context of Landau-Ginzburg orbifolds. Whenever the polynomial ring $\mathbb{C}\left[X_{1}, \ldots, X_{n}\right]$ carries an action of a finite group $G_{W}$ which leaves a polynomial $W$ invariant, the Landau-Ginzburg model defined by $W$ can be orbifolded by $G_{W}$ leading to a new $2 \mathrm{~d}$ TQFT which by abuse of notation we denote by $W / G_{W}[22]$.

Now let $V \in \mathbb{C}\left[X_{1}, \ldots, X_{n}\right]$ and $W \in \mathbb{C}\left[Y_{1}, \ldots, Y_{m}\right]$ be two superpotentials and $G_{V}$ and $G_{W}$ orbifold groups. Then B-type defects between the respective LG orbifolds can be described by $G=G_{V} \times G_{W}$-equivariant matrix factorizations of $V-W[1,23,24]$. These are matrix factorizations $D: W \rightarrow V$ as before, which are additionally equipped with a representation $\rho_{D}$ of $G$. The latter has to be compatible with the module structure on $D$ and has to commute with $\mathrm{d}_{D}$. Denoting by $\rho$ the representation of $G=G_{V} \times G_{W}$ on the combined polynomial ring $S=\mathbb{C}\left[X_{1}, \ldots, X_{n}, Y_{1}, \ldots, Y_{m}\right]$ this means that for all $g \in G$

$$
\begin{aligned}
\rho_{D}(g)(s \cdot p) & =\rho(g)(s) \cdot \rho_{D}(g)(p), \quad \forall s \in S, p \in D=D_{0} \oplus D_{1}, \\
\rho_{D}(g) \circ \mathrm{d}_{D} & =\mathrm{d}_{D} \circ \rho_{D}(g) .
\end{aligned}
$$

Given two equivariant matrix factorizations $D, D^{\prime}: W \rightarrow V$, the complex $\operatorname{Hom}_{S}\left(D, D^{\prime}\right)$ carries an action of $G=G_{V} \times G_{W}$ which commutes with the differential d, inducing a representation on the homology $H_{\mathrm{d}}^{*}\left(\operatorname{Hom}_{S}\left(D, D^{\prime}\right)\right)$. The space of defect-changing fields in the orbifold theory is then given by the $G$-invariant part $\operatorname{Hom}^{G}\left(D, D^{\prime}\right)=\left(H_{\mathrm{d}}^{*}\left(\operatorname{Hom}_{S}\left(D, D^{\prime}\right)\right)\right)^{G}$. The operator product of defect-changing fields is again just composition of homomorphisms.

Defect fusion carries over from the unorbifolded LG models by taking invariant parts. More precisely, let $U \in \mathbb{C}\left[X_{1}, \ldots, X_{m}\right], V \in \mathbb{C}\left[Y_{1}, \ldots, Y_{n}\right]$ and $W \in \mathbb{C}\left[Z_{1}, \ldots, Z_{o}\right]$ be polynomials invariant under actions of groups $G_{U}, G_{V}, G_{W}$ on the respective polynomial rings. And let $D: W \rightarrow V$ and $D^{\prime}: V \rightarrow U$ be $G_{W} \times G_{V^{-}}$, respectively $G_{V} \times G_{U^{-}}$ equivariant matrix factorizations. Then the tensor product $D^{\prime} \otimes D$ is a $G_{U} \times G_{V} \times G_{W^{-}}$ equivariant matrix factorization of $U-W$. Fusion of the defects in the orbifold theory is then given by the $G_{V}$-invariant part $D^{\prime} \otimes_{G_{V}} D:=\left(D^{\prime} \otimes D\right)^{G_{V}}$ of $D^{\prime} \otimes D$, which is of course $G_{U} \times G_{W^{-}}$equivariant.

Adjunction of defects $D$ in the orbifold theory is given by adjunction (4.1) in the underlying unorbifolded theory, where however the $G$-action on the adjoints is twisted. This can be seen in a systematic way in the generalized orbifold construction $[4,5,7,8]$ which offers a completely general framework to describe orbifold theories using defects in the underlying unorbifolded theory. We outline the generalized orbifold procedure in appendix B, and in particular spell out the formula for adjoints. 


\subsection{Defects $\mathcal{M}_{d} / \mathbb{Z}_{d} \rightarrow \mathcal{M}_{d^{\prime}} / \mathbb{Z}_{d^{\prime}}$}

For the case of Landau-Ginzburg orbifolds $\mathcal{M}_{d} / \mathbb{Z}_{d}$ the discussion simplifies somewhat. An element $a \in \mathbb{Z}_{d}$ of the orbifold group acts on the chiral field $X$ by $X \mapsto e^{\frac{2 \pi i a}{d}} X$.

A defect $D: X^{d} / \mathbb{Z}_{d} \rightarrow Z^{d^{\prime}} / \mathbb{Z}_{d^{\prime}}$ is given by a $G=\mathbb{Z}_{d^{\prime}} \times \mathbb{Z}_{d^{-}}$equivariant matrix factorization of $Z^{d^{\prime}}-X^{d}$. Since $G$ is commutative, its representations on $D$ can be specified by $G$-gradings or -charges of the generators of the free $S=\mathbb{C}[Z, X]$-module $D=D_{0} \oplus D_{1}$. We will indicate them in square brackets and specify a $G$-equivariant matrix factorizations as

$$
D: S^{M}\left(\begin{array}{c}
{\left[l_{M}, r_{M}\right]} \\
{\left[l_{M+1}, r_{M+1}\right]} \\
\vdots \\
{\left[l_{2 M-1}, r_{2 M-1}\right]}
\end{array}\right) \underset{\mathrm{d}_{D 0}}{\stackrel{\mathrm{d}_{D 1}}{\rightleftarrows}} S^{M}\left(\begin{array}{c}
{\left[l_{0}, r_{0}\right]} \\
{\left[l_{1}, r_{1}\right]} \\
\vdots \\
{\left[l_{M-1}, r_{M-1}\right]}
\end{array}\right)
$$

cf. [1] for more details. Adjoints then take the form, cf. appendix C.4,

$$
\begin{gathered}
D^{\dagger}: S^{M}\left(\begin{array}{c}
{\left[-r_{0}+1,-l_{0}\right]} \\
{\left[-r_{1}+1,-l_{1}\right]} \\
\vdots \\
{\left[-r_{M-1}+1,-l_{M-1}\right]}
\end{array}\right) \stackrel{\mathrm{d}_{D 1}^{T}}{\underset{-\mathrm{d}_{D 0}^{T}}{\leftrightarrows}} S^{M}\left(\begin{array}{c}
{\left[-r_{M}+1,-l_{M}\right]} \\
{\left[-r_{M+1}+1,-l_{M+1}\right]} \\
\vdots \\
{\left[-r_{2 M-1}+1,-l_{2 M-1}\right]}
\end{array}\right) \\
{ }^{\dagger} D: S^{M}\left(\begin{array}{c}
{\left[-r_{0},-l_{0}+1\right]} \\
{\left[-r_{1},-l_{1}+1\right]} \\
\vdots \\
{\left[-r_{M-1},-l_{M-1}+1\right]}
\end{array}\right) \underset{\left[-\mathrm{d}_{D 0}^{T}\right.}{\stackrel{\mathrm{d}_{D 1}^{T}}{\leftrightarrows}} S^{M}\left(\begin{array}{c}
{\left[-r_{M}+1\right]} \\
{\left[-r_{M+1},-l_{M+1}+1\right]} \\
\vdots \\
{\left[-r_{2 M-1},-l_{2 M-1}+1\right]}
\end{array}\right)
\end{gathered}
$$

Note that left adjoints differ from right adjoints by a shift in $G$-charges by $[-1,1]$. We write ${ }^{\dagger} D=D^{\dagger}\{[-1,1]\}$.

An important example is the identity defect $I_{d}: X^{d} / \mathbb{Z}_{d} \rightarrow Z^{d} / \mathbb{Z}_{d}$ which is represented by the following $\mathbb{Z}_{d} \times \mathbb{Z}_{d}$-equivariant matrix factorization (cf. appendix C.6)

$$
I_{d}: S^{d}\left(\begin{array}{c}
{[1,0]} \\
{[2,-1]} \\
{[3,-2]} \\
\vdots
\end{array}\right) \stackrel{\left(\begin{array}{ccccc}
Z & 0 & \ldots & 0 & -X \\
-X & Z & & & \\
0 & -X & Z & & \\
\vdots & & \ddots & \ddots & \\
0 & & & -X & Z
\end{array}\right)}{\rightleftarrows} S^{d}\left(\begin{array}{c}
{[0,0]} \\
{[1,-1]} \\
{[2,-2]} \\
\vdots
\end{array}\right) .
$$

One easily reads off that this defect is self-adjoint, i.e. $I_{d}^{\dagger} \cong I_{d} \cong{ }^{\dagger} I_{d}$. 


\subsection{RG defects in LG orbifolds}

As alluded to above, Landau-Ginzburg orbifolds $\mathcal{M}_{d} / \mathbb{Z}_{d}$ exhibit relevant perturbations by twisted chiral fields. The corresponding RG flows drive the theory from $\mathcal{M}_{d} / \mathbb{Z}_{d}$ in the UV to another orbifold $\mathcal{M}_{d^{\prime}} / \mathbb{Z}_{d^{\prime}}$ with $d^{\prime}<d$ in the IR. The associated RG defects have been constructed in [1]. They preserve B-type supersymmetry and can therfore be described by

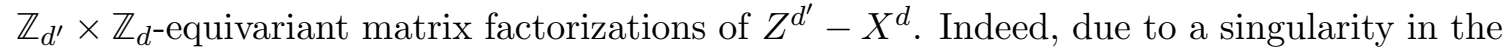
parameter space, there are different flows from $\mathcal{M}_{d} / \mathbb{Z}_{d}$ to $\mathcal{M}_{d^{\prime}} / \mathbb{Z}_{d^{\prime}}$. The corresponding RG defects $R=R\left(m, n_{0}, \ldots, n_{d^{\prime}-1}\right)$ are specified by $m \in \mathbb{Z}_{d}$, and integers $n_{0}, \ldots, n_{d^{\prime}-1} \geq 1$, such that $n_{0}+\ldots+n_{d^{\prime}-1}=d$. They are represented by matrix factorizations

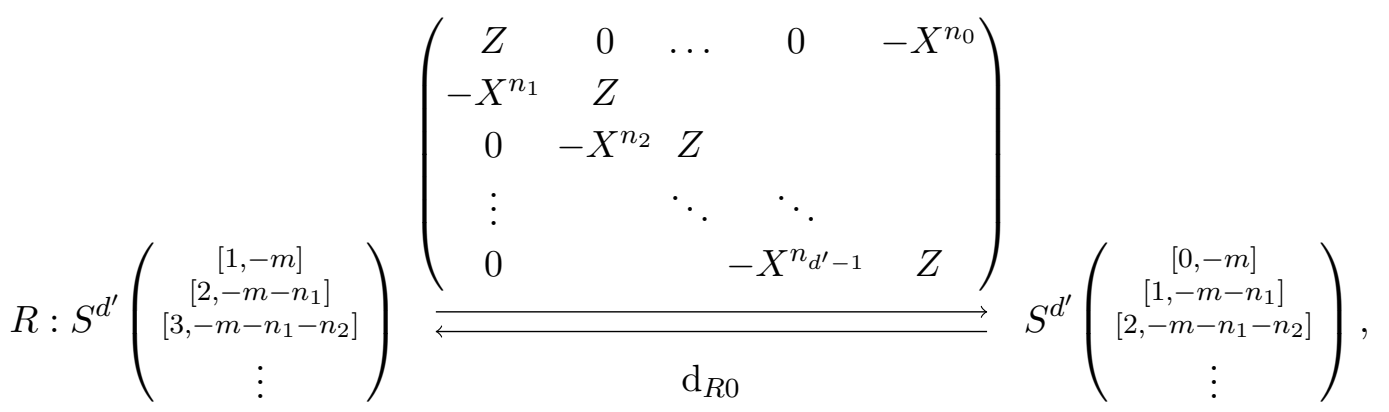

where, $S=\mathbb{C}[X, Z]$. For more details see [1]. In the following we will sometimes take the subscripts of the $n_{i}$ to be elements in $\mathbb{Z}_{d^{\prime}}$ by defining $n_{i+z d^{\prime}}=n_{i}$ for all $z \in \mathbb{Z}$.

Using this concrete realization of RG defects, one can now explicitly carry out the construction outlined in section 3 and represent the LG orbifolds $\mathcal{M}_{d^{\prime}} / \mathbb{Z}_{d^{\prime}}$ in $\mathcal{M}_{d} / \mathbb{Z}_{d}$ for any $d^{\prime}<d$. In order to construct the respective projection defects, we need right and left adjoints of the defects $R$, which can easily be read off from formula (4.2). They are given by

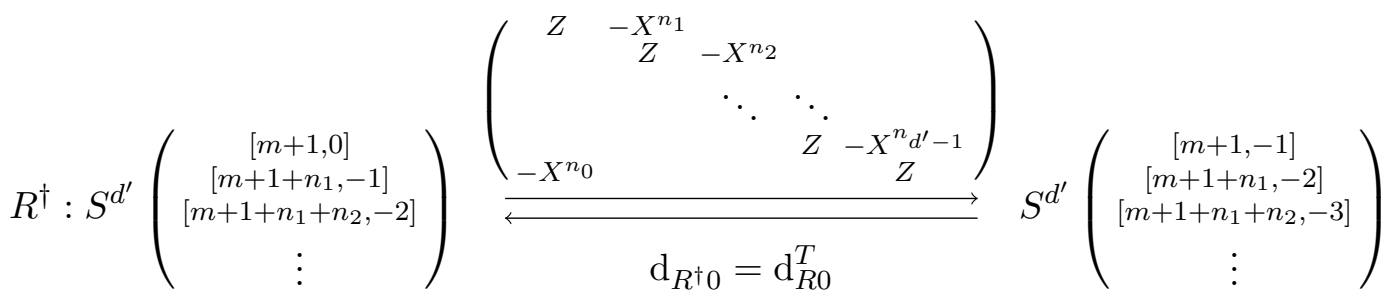

and

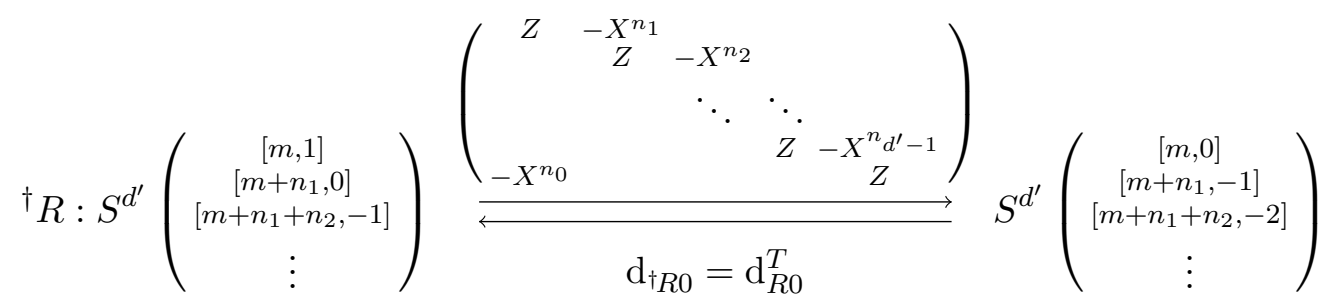

A straight-forward calculation presented in appendix D.1 then shows that indeed

$$
\begin{aligned}
& R \otimes_{\mathbb{Z}_{d}} R^{\dagger} \cong I_{d^{\prime}} \\
& R \otimes_{\mathbb{Z}_{d}}{ }^{\dagger} R \cong I_{d^{\prime}},
\end{aligned}
$$


i.e. the defects $R$ are indeed of RG type. Fusion in the opposite order yields the respective projection defects (see appendix D.2 for the explicit calculation). For the unital projection defect $P=R^{\dagger} \otimes_{\mathbb{Z}_{d^{\prime}}} R$ one obtains

$$
P: S^{d^{\prime}}\left(\begin{array}{c}
{[m+1,-m]} \\
{\left[m+1+n_{1},-m-n_{1}\right]} \\
{\left[m+1+n_{1}+n_{2},-m-n_{1}-n_{2}\right]} \\
\vdots \\
{\left[m+1+\sum_{l=1}^{d^{\prime}-1} n_{l},-m-\sum_{l=1}^{d^{\prime}-1} n_{l}\right]}
\end{array}\right) \stackrel{\mathrm{d}_{P 1}}{\rightleftarrows} S^{d^{\prime} 0}\left(\begin{array}{c}
{\left[m+1+\sum_{l=1}^{d^{\prime}-1} n_{l},-m\right]} \\
{\left[m+1,-m-n_{1}\right]} \\
{\left[m+1+n_{1},-m-n_{1}-n_{2}\right]} \\
\vdots \\
{\left[m+1+\sum_{l=1}^{d^{\prime}-2} n_{l},-m-\sum_{l=1}^{d^{\prime}-1} n_{l}\right]}
\end{array}\right),
$$

where

$$
\mathrm{d}_{P 1}=\left(\begin{array}{ccccc}
Z^{n_{0}} & 0 & \cdots & 0 & -X^{n_{0}} \\
-X^{n_{1}} & Z^{n_{1}} & & & \\
0 & -X^{n_{2}} & Z^{n_{2}} & & \\
\vdots & & \ddots & \ddots & \\
0 & & & -X^{n_{d^{\prime}-1}} & Z^{n_{d^{\prime}-1}}
\end{array}\right) .
$$

The counital projection defects $P^{\prime}={ }^{\dagger} R \otimes_{\mathbb{Z}_{d^{\prime}}} R$ is given by the left adjoint $P^{\prime}={ }^{\dagger} P$ of $P$.

The morphism

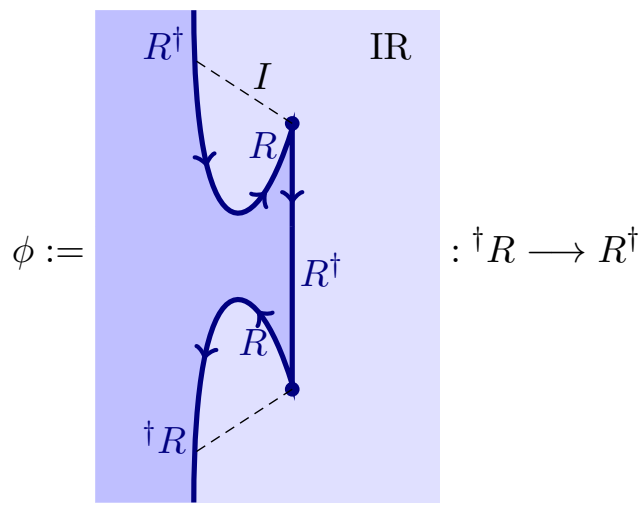

which is used to close right $R$-loops can also be determined explicitly. It is not hard to see that it is given by

$$
\phi=\left(\begin{array}{cc}
\phi_{0} & 0 \\
0 & \phi_{1}
\end{array}\right) \quad \text { with } \quad \phi_{0}=\phi_{1}=\left(\begin{array}{cccc}
0 & X^{n_{1}-1} & \\
& \ddots & \ddots & \\
& & \ddots & X^{n_{d^{\prime}}-1} \\
& & & \\
X^{n_{0}-1} & & & 0
\end{array}\right) .
$$

\subsection{Representing $\mathcal{M}_{d^{\prime}} / \mathbb{Z}_{d^{\prime}}$ in $\mathcal{M}_{d} / \mathbb{Z}_{d}$ for $d^{\prime}<d$}

The projection defects constructed from $R G$ defects in the previous section can now be used to represent Landau-Ginzburg orbifolds $\mathcal{M}_{d^{\prime}} / \mathbb{Z}_{d^{\prime}}$ in orbifolds $\mathcal{M}_{d} / \mathbb{Z}_{d}$ for $d^{\prime}<d$.

Bulk Hilbert space. The orbifolds $\mathcal{M}_{d^{\prime}} / \mathbb{Z}_{d^{\prime}}$ only possess a single bulk chiral field, namely the identity field. Therefore, the bulk Hilbert space in the B-twisted model is trivial, it just contains the vaccuum. One easily checks, that this is also true for $\operatorname{Hom}(P, P)$. Hence, 
the bulk Hilbert space of $\mathcal{M}_{d^{\prime}} / \mathbb{Z}_{d^{\prime}}$ agrees with the space of defect fields on the projection defect in $\mathcal{M}_{d} / \mathbb{Z}_{d}{ }^{15}$

Boundary conditions. Next, we demonstrate how to represent the boundary conditions of $\mathcal{M}_{d^{\prime}} / \mathbb{Z}_{d^{\prime}}$ as $P$-invariant boundary conditions in the models $\mathcal{M}_{d} / \mathbb{Z}_{d}$.

Elementary left boundary conditions in a theory $\mathcal{M}_{d} / \mathbb{Z}_{d}$ are represented by the $\mathbb{Z}_{d^{-}}$ equivariant matrix factorizations

$$
B_{k, N}^{d}: \mathbb{C}[X]([N+k]) \underset{-X^{d-k}}{\longleftarrow} \mathbb{C}[X]([N])
$$

of $-X^{d}$, where $k \in\{1, \ldots, d-1\}$ and $N \in \mathbb{Z}_{d}$.

As is shown in appendix D.3, a UV boundary condition $B_{\mathrm{UV}}=B_{k, N}^{d}$ is invariant under fusion with $P$, i.e. $B_{\mathrm{UV}} \otimes P \cong B_{\mathrm{UV}}$ iff

$$
\begin{aligned}
k & =n_{i}+\ldots+n_{i-l} \\
\text { and } \quad N & =\left[-m-\sum_{a=1}^{i} n_{a}\right]
\end{aligned}
$$

for an $i \in \mathbb{Z}_{d^{\prime}}$ and an $l \in\left\{0, \ldots, d^{\prime}-2\right\}$. These are of course nothing but the lifts $B_{\mathrm{IR}} \otimes_{\mathbb{Z}_{d^{\prime}}} R$ of IR boundary conditions to the UV. Namely, for $B_{\mathrm{IR}}=B_{l, M}^{d^{\prime}}$ one finds [1]

$$
B_{\mathrm{IR}} \otimes_{\mathbb{Z}_{d^{\prime}}} R=B_{\left(n_{-M-l+1}+\ldots+n_{-M}\right),\left(-m-\sum_{a=1}^{-M} n_{a}\right)}^{d} .
$$

IR symmetries. The Landau-Ginzburg orbifold model $\mathcal{M}_{d^{\prime}} / \mathbb{Z}_{d^{\prime}}$ exhibits a $\mathbb{Z}_{d^{\prime}}$-symmetry. The action of an element $a \in \mathbb{Z}_{d^{\prime}}$ on the theory is described by the symmetry defect ${ }_{a} I_{d^{\prime}}=I_{d^{\prime}}\{[a, 0]\} \cong I_{d^{\prime}}\{[0,-a]\}$ obtained by shifting the charges of the identity defect $I_{d^{\prime}}$ by $[a, 0]$ or equivalently by $[0,-a]$. These defects fuse according to the group multiplication in the symmetry group $\mathbb{Z}_{d^{\prime}}$ :

$$
{ }_{a} I_{d^{\prime}} \otimes_{\mathbb{Z}_{d^{\prime}} b} I_{d^{\prime}}={ }_{a+b} I_{d^{\prime}}, \quad \text { for } \quad a, b \in \mathbb{Z}_{d^{\prime}} .
$$

As any IR defects, they lift into the UV theory $\mathcal{M}_{d} / \mathbb{Z}_{d}$ by fusion with RG defects

$$
{ }_{a} I_{d^{\prime}} \longmapsto R^{\dagger} \otimes_{\mathbb{Z}_{d^{\prime}} a} I_{d^{\prime}} \otimes_{\mathbb{Z}_{d^{\prime}}} R=:{ }_{a} P .
$$

These lifted defects also fuse according to multiplication in the symmetry group, i.e. ${ }_{a} P \otimes_{\mathbb{Z}_{d}}$ ${ }_{b} P={ }_{a+b} P$, and therefore give a realization of the IR symmetry in the UV. The neutral element of the group however lifts to the defect ${ }_{0} P=P$ and not to the identity defect in the UV. The lifted IR symmetries are therefore not invertible in the full UV theory, and hence are not symmetries of the UV theory.

\footnotetext{
${ }^{15}$ Since the bulk Hilbert spaces are trivial, this is not that interesting. However, there is a way to describe also the twisted chiral fields in the B-twisted LG orbifolds $\mathcal{M}_{d} / \mathbb{Z}_{d}$. Namely, being orbifold twist fields, they can be realized as defect changing fields between symmetry defects. This realization then lifts from IR to UV using projection defects, i.e. one can realize the twisted chiral fields in $\mathcal{M}_{d^{\prime}} / \mathbb{Z}_{d^{\prime}}$ by defect changing fields in $\mathcal{M}_{d} / \mathbb{Z}_{d}$.
} 
The explicit form of ${ }_{a} P$ can be easily derived by means of a slight variation of the calculation of $P$ as carried out in appendix D.4. The result is

$$
{ }_{a} P: S^{d^{\prime}}\left(\begin{array}{c}
{\left[m+1,-m-\sum_{j=1}^{-a} n_{j}\right]} \\
{\left[m+1+n_{1},-m-\sum_{j=1}^{1-a} n_{j}\right]} \\
{\left[m+1+n_{1}+n_{2},-m-\sum_{j=1}^{2-a} n_{j}\right]} \\
\vdots \\
{\left[m+1+\sum_{l=1}^{d^{\prime}-1} n_{l},-m-\sum_{l=1}^{d^{\prime}-1} n_{l}\right]}
\end{array}\right) \stackrel{\mathrm{d}_{P 1}}{\rightleftarrows} S^{d^{\prime} 0}\left(\begin{array}{c}
{\left[m+1+\sum_{l=1}^{d^{\prime}-1} n_{l},-m-\sum_{j=1}^{-a} n_{j}\right]} \\
{\left[m+1,-m-\sum_{j=1}^{1-a} n_{j}\right]} \\
{\left[m+1+n_{1},-m-\sum_{j=1}^{2-a} n_{j}\right]} \\
\vdots \\
{\left[m+1+\sum_{l=1}^{d^{\prime}-2} n_{l},-m-\sum_{l=1}^{d^{\prime}-1-a} n_{l}\right]}
\end{array}\right),
$$

where

$$
\mathrm{d}_{P 1}=\left(\begin{array}{ccccc}
Z^{n_{0}} & 0 & \cdots & 0 & -X^{n_{0-a}} \\
-X^{n_{1-a}} & Z^{n_{1}} & & & \\
0 & -X^{n_{2-a}} & Z^{n_{2}} & & \\
\vdots & & \ddots & \ddots & \\
0 & & & -X^{n_{d^{\prime}-1-a}} & Z^{n_{d^{\prime}-1}}
\end{array}\right) .
$$

For $a=0$, this is the matrix factorization describing $P$. The lifted IR symmetry defects ${ }_{a} P$ are obtained from it by shifting the exponents of $X$ by $a$ steps while keeping left $\mathbb{Z}_{d}$-charges fixed and adapting the right ones accordingly.

\subsection{The limit $d \rightarrow \infty$}

As discussed in the beginning of this section, the RG flows between LG orbifolds $\mathcal{M}_{d} / \mathbb{Z}_{d}$ are nothing but the mirror versions of flows between LG models $\mathcal{M}_{d}$ generated by deformations of the superpotentials $W=X^{d}$ by lower degree polynomials. Indeed, all the models $\mathcal{M}_{d^{\prime}}$ can be obtained as perturbations of the free chiral field theory $(W=0)$ by superpotential deformations. Thus, employing our procedure provides a representation of all the models $\mathcal{M}_{d^{\prime}}$ inside the theory of a free chiral field, which can be thought of as the limit $\mathcal{M}_{\infty}=$ $\lim _{d \rightarrow \infty} \mathcal{M}_{d}$ of the models $\mathcal{M}_{d}$.

In order to make this more explicit we again take the mirror perspective. The representation of the respective RG defects in terms of matrix factorizations then allows us to explicitly realize all the LG orbifolds $\mathcal{M}_{d^{\prime}} / \mathbb{Z}_{d^{\prime}}$ by means of projection defects in the theory of the free twisted chiral field. The latter can be described as the limit $\mathcal{M}_{\infty} / \mathbb{Z}_{\infty}=\lim _{d \rightarrow \infty} \mathcal{M}_{d} / \mathbb{Z}_{d}$ and can be thought of as a $\mathrm{U}(1)$-equivariant version of the free chiral field.

RG defects between $\mathcal{M}_{d^{\prime}} / \mathbb{Z}_{d^{\prime}}$ and $\mathcal{M}_{\infty} / \mathbb{Z}_{\infty}$ can be obtained as limits of the RG defects (4.3) representing flows $\mathcal{M}_{d} / \mathbb{Z}_{d} \rightarrow \mathcal{M}_{d^{\prime}} / \mathbb{Z}_{d^{\prime}}$, where one $n_{i}$ is sent to $\infty$ while the others are kept fixed. Since $d=\sum_{i} n_{i}$, then also $d \rightarrow \infty$. Indeed, we can choose $n_{0} \rightarrow \infty$ and compensate for this choice by allowing a shift of the charges of $R$ by $[k, 0], k \in \mathbb{Z}_{d^{\prime}}$. In the limit, entries $X^{n_{0}}$ in the matrix factorization have to be replaced by 0 , and the $\mathbb{Z}_{d}$-equivariance turns into a $\mathrm{U}(1)$-equivariance. This way, one obtains the $\mathbb{Z}_{d^{\prime}} \times \mathrm{U}(1)$-equivariant matrix factorizations

$$
R_{\infty}: S^{d^{\prime}}\left(\begin{array}{c}
{[k+1,-m]} \\
{\left[k+2,-m-n_{1}\right]} \\
{\left[k+3,-m-n_{1}-n_{2}\right]} \\
\vdots
\end{array}\right) \stackrel{\mathrm{d}_{R 1}}{\rightleftarrows \mathrm{d}_{R 0}} S^{d^{\prime}}\left(\begin{array}{c}
{[k,-m]} \\
{\left[k+1,-m-n_{1}\right]} \\
{\left[k+2,-m-n_{1}-n_{2}\right]} \\
\vdots
\end{array}\right)
$$


of $Z^{d^{\prime}}$. They are specified by integers $m \in \mathbb{Z}, n_{1}, \ldots, n_{d^{\prime}-1} \in \mathbb{N}$ and $k \in \mathbb{Z}_{d^{\prime}}$. The maps are given by

$$
\begin{aligned}
& \mathrm{d}_{R 1}=\left(\begin{array}{ccccc}
Z & 0 & \cdots & 0 & 0 \\
-X^{n_{1}} & Z & & & \\
0 & -X^{n_{2}} & Z & & \\
\vdots & & \ddots & \ddots & \\
0 & & & -X^{n_{d^{\prime}-1}} & Z
\end{array}\right) \\
& \mathrm{d}_{R 0}=\left(\begin{array}{cccc}
Z^{d^{\prime}-1} & 0 & 0 & \cdots \\
Z^{d^{\prime}-2} X^{n_{1}} & Z^{d^{\prime}-1} & 0 & \cdots \\
Z^{d^{\prime}-3} X^{n_{1}+n_{2}} & Z^{d^{\prime}-2} X^{n_{2}} & Z^{d^{\prime}-1} & \ddots \\
Z^{d^{\prime}-4} X^{n_{1}+n_{2}+n_{3}} & Z^{d^{\prime}-3} X^{n_{2}+n_{3}} & Z^{d^{\prime}-2} X^{n_{3}} & \ddots \\
\vdots & \vdots & \vdots & \ddots
\end{array}\right) .
\end{aligned}
$$

These matrix factorizations represent RG defects between $\mathcal{M}_{d^{\prime}} / \mathbb{Z}_{d^{\prime}}$ and $\mathcal{M}_{\infty} / \mathbb{Z}_{\infty}$. Indeed $R_{\infty} \otimes R_{\infty}^{\dagger} \cong I_{\mathrm{IR}}$ as is spelled out in appendix D.5.

In explicit calculations, it is not difficult to see that fusion commutes with the limit $d \rightarrow \infty$, at least as long as the theory squeezed between the defects is kept fixed in the limit. In particular, the limit $d \rightarrow \infty$ of projection defects is the fusion $P_{\infty}=R_{\infty}^{\dagger} \otimes R_{\infty}$ of the limit of RG defects. The projection defect realizing $\mathcal{M}_{d^{\prime}} / \mathbb{Z}_{d^{\prime}}$ within the limit theory $\mathcal{M}_{\infty} / \mathbb{Z}_{\infty}$ takes the form

$$
P_{\infty}: S^{d^{\prime}}\left(\begin{array}{c}
{[m+1,-m]} \\
{\left[m+1+n_{1},-m-n_{1}\right]} \\
{\left[m+1+n_{1}+n_{2},-m-n_{1}-n_{2}\right]} \\
\vdots \\
{\left[m+1+\sum_{l=1}^{d^{\prime}-1} n_{l},-m-\sum_{l=1}^{d^{\prime}-1} n_{l}\right]}
\end{array}\right) \stackrel{\mathrm{d}_{P 1}}{\rightleftarrows} S^{d^{\prime} 0}\left(\begin{array}{c}
{\left[m+1+\sum_{l=1}^{d^{\prime}-1} n_{l},-m\right]} \\
{\left[m+1,-m-n_{1}\right]} \\
{\left[m+1+n_{1},-m-n_{1}-n_{2}\right]} \\
\vdots \\
{\left[m+1+\sum_{l=1}^{d^{\prime}-2} n_{l},-m-\sum_{l=1}^{d^{\prime}-1} n_{l}\right]}
\end{array}\right)
$$

where $S=\mathbb{C}[Z, X]$ and

$$
\mathrm{d}_{P 1}=\left(\begin{array}{ccccc}
0 & & & & 0 \\
-X^{n_{1}} & Z^{n_{1}} & & & \\
& -X^{n_{2}} & Z^{n_{2}} & & \\
& & \ddots & \ddots & \\
& & & -X^{n_{d^{\prime}-1}} & Z^{n_{d^{\prime}}-1}
\end{array}\right) .
$$

\section{Conclusion}

We conclude with a list of questions for future investigation.

- It would be interesting to apply the construction outlined in this paper to other examples of RG flows and to find representations of more elaborate 2d TQFTs in free theories by means of projectors.

The treatment of flows between LG orbifolds discussed in section 4 easily carries over to flows between orbifolds of free chiral field theories. The latter theories can be 
obtained from LG orbifolds by setting the superpotentials to zero. The respective RG defects can be described in terms of matrix factorizations and have been worked out in [25]. Somewhat more interesting examples are the bulk flows described in terms of gauged linear sigma models in [26] and [27, 28]. In all these examples, the flows are triggered by twisted chiral perturbations yielding $R G$ defects preserving B-type supersymmetry. It would of course be interesting to apply this method to chiral perturbations as well. Unfortunately much less is known about A-type defects.

- In the example of LG orbifolds $\mathcal{M}_{d} / \mathbb{Z}_{d}$ one could explicitly compare the bulk flows studied in section 4.3 with the corresponding flows of the identity defects. While the identity defects can be described by means of matrix factorizations, the respective relevant perturbations are by twisted chiral fields. Such perturbations are not as easily treated in the matrix factorization framework as defect perturbations by chiral fields such as the ones discussed in [29]. In the case at hand however, the twisted chiral fields generating the perturbations are orbifold twist fields. As such, they do have a representation in the matrix factorization framework as defect changing fields. This possibly allows for an explicit analysis of the respective defect perturbations in this case. Hence, it might be possible to work out in this concrete example how the projection defects $P$ associated to bulk flows arise as perturbations of identity defects.

- We have argued that bulk RG flows can be interpreted as flows on the identity defect of the UV theory. Now, bulk flows between $N=2$ SCFTs give rise to $t t^{*}$ equations $[30,31]$. It would be very interesting to see, whether these equations also have a natural interpretation in terms of the flows on the identity defect.

- The construction described in this paper heavily relies on topological covariance. So an obvious question is whether it has any bearing on non-topological QFTs (beyond topologically twisted supersymmetric theories). One can of course perturb the identity defect in non-topological QFTs. Also, RG defects exist in more general theories (see e.g. [2] for an example). However, fusion of non-topological defects is singular in general. Still, at least in some cases it is possible to define a reasonable notion of fusion [32, 33], so that defects $P$ can be constructed from RG defects. The role of these defects is less clear, but it would be very interesting to study them in examples. Perhaps they are related to the line operators appearing in the context of integrable perturbations of conformal field theories [34-38].

- While the discussion in this paper is restricted to 2d TQFTs, we expect that bulk perturbations of TQFTs can be described by means of codimension-one projection defects in any dimension. Indeed, the generalized orbifold construction has been extended from dimension two to higher dimensions in [39]. It would be very interesting to apply the methods described in this paper to higher-dimensional TQFTs. 


\section{Acknowledgments}

FK thanks Friedrich-Naumann-Stiftung and the Chinese Scholarship Council for supporting him throughout this project. DR's work is supported by hits. For ease of readability accross the literature, we have borrowed the defect coloring scheme from [8].

\section{A Properties of projection defects}

\section{A.1 IR bulk fields in the UV}

Here, we show that if a projection defect $P$ in the $\mathrm{UV}$ factorizes as $P \cong R^{\dagger} \otimes R$ with $R \otimes R^{\dagger} \cong I_{\mathrm{IR}}$, then $P$-bimodule morphisms on $P$ are one-to-one with IR bulk fields $\left(I_{\mathrm{IR}^{-}}\right.$ bimodule morphisms of $\left.I_{\mathrm{IR}}\right)$. The latter are mapped into the UV by the homomorphism

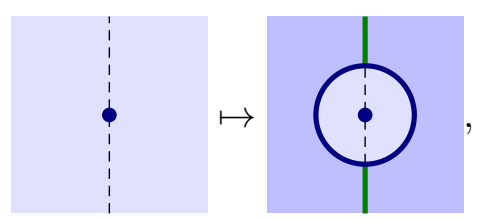

where the junctions are given by the isomorphisms $P \cong R^{\dagger} \otimes R$. (To keep the notation light, we refrain from putting arrows on RG defects. We mark UV and IR theory by dark, respectively light background.) By the projection property the right hand side is a $P$-bimodule morphism of $P$. We claim that the inverse to the homomorphism (A.1) is given by

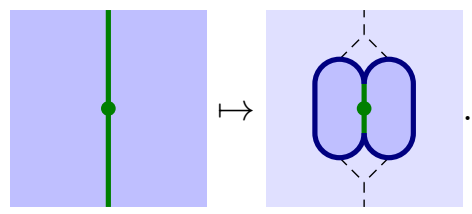

Let us first check that the composition IR $\rightarrow$ UV $\rightarrow$ IR evaluates to the identity on IR bulk fields: ${ }^{16}$

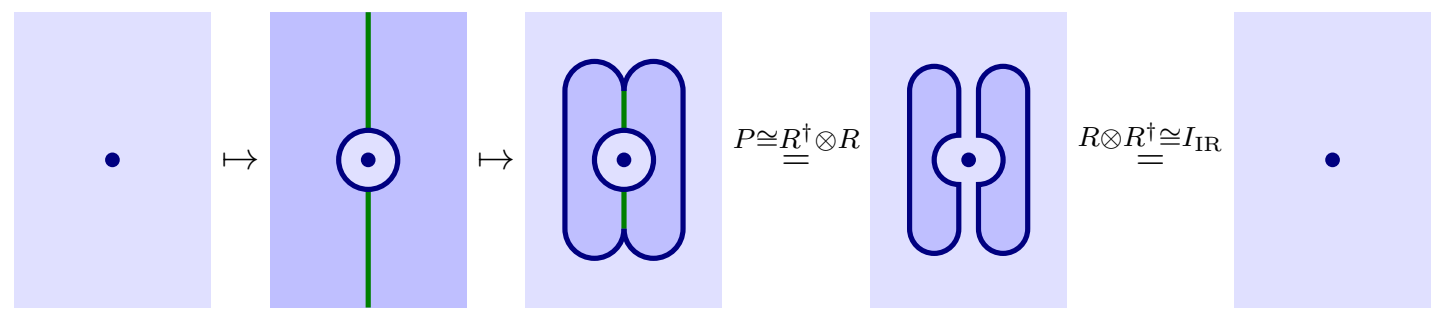

Similarly, the composition UV $\rightarrow$ IR $\rightarrow$ UV is the identity on $P$-bimodule morphisms of $P:$

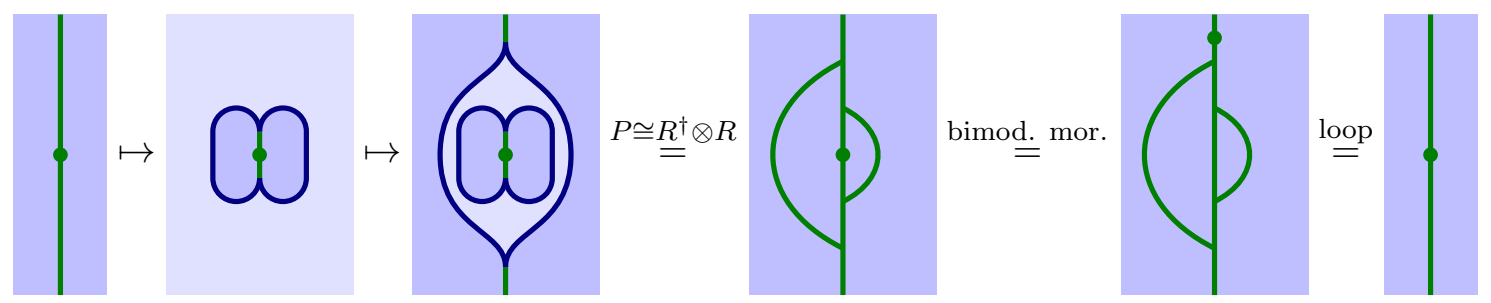

\footnotetext{
${ }^{16}$ For readability, $I_{\mathrm{IR}}$ has been omitted.
} 
Therefore we have established that the map (A.1) is an isomorphism from the space of IR bulk fields to the space of $P$-bimodule morphisms of $P$.

\section{A.2 Bimodule equal bicomodule morphisms}

For any projection defect, bimodule morphisms over itself are automatically cobimodule morphisms and vice versa. In other words, the two types of morphisms are one-to-one:
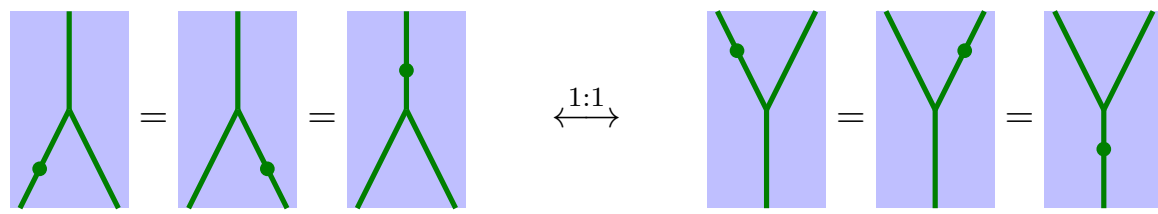

This is easy to see. A bimodule morphism for example (left-hand side above) automatically obeys

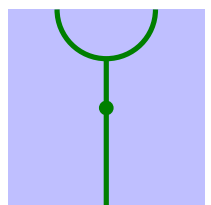

$\stackrel{\text { loop }}{=}$

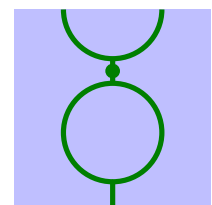

bimod. mor.
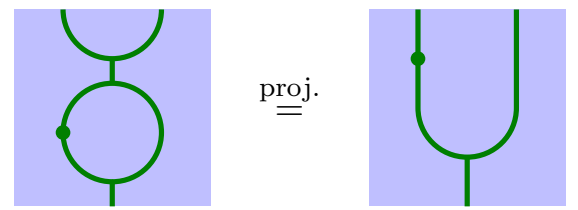

bimod. mor.

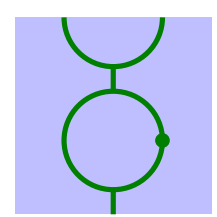

$\stackrel{\text { proj. }}{=}$

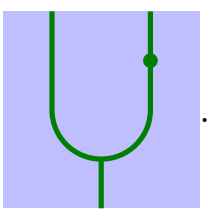

The argument that bicomodule morphisms also respect the bimodule structure follows by turning the diagrams above upside down.

Indeed, if the projection defect $P$ comes with a unit then all morphisms of $P$ automatically respect the bicomodule structure, and by the above also the bimodule structure on $P$. (This easily follows from the projection property.) Hence, in this case all morphisms of $P$ are bimodule morphisms and bicomodule morphisms. The same is true if $P$ has a counit.

\section{A.3 $P$-modules $B$ and $B \otimes P \cong B$}

In this appendix we show that for a projection defect $P$, any left boundary condition $B$ is a right $P$-module if and only if $B \otimes P \cong B$. This in particular means that left IR boundary conditions can be represented by left boundary conditions $B$ in the UV which are invariant under fusion with $P$, i.e. $B \otimes P \cong B$. This statement extends to right boundaries and defects.

First, a left $P$-module (whose comodule structure is induced by the unit on $P$ ) obeys $B \otimes P \cong B:$

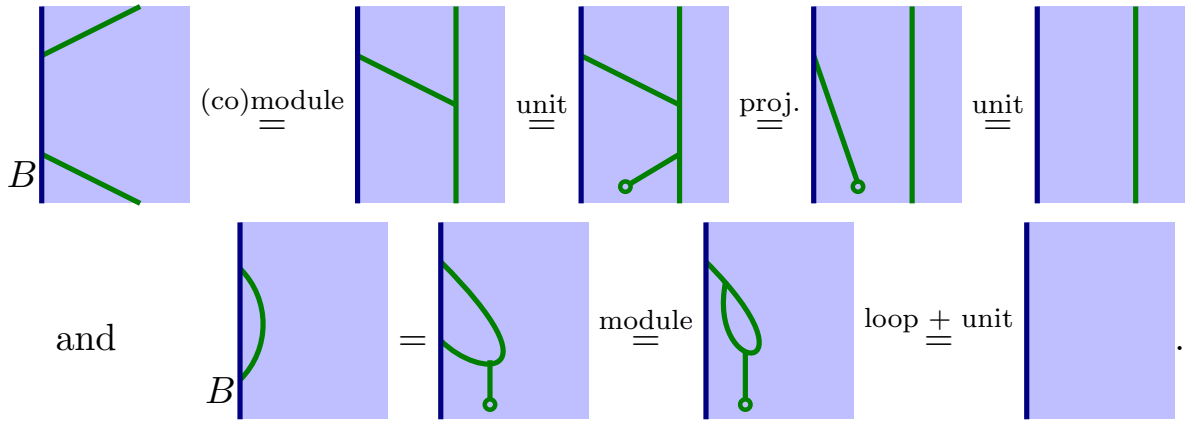


If on the other hand a left boundary condition $B$ satisfies $B \otimes P \cong B, B$ inherits the $P$-module structure of $P$ itself. Namely, there are junctions $B \otimes P \rightarrow B$ and $B \rightarrow B \otimes P$ such that
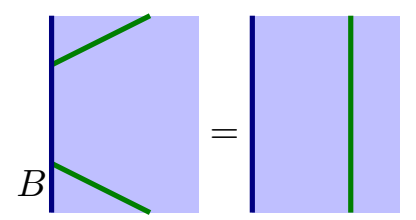

and

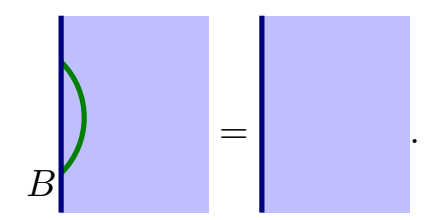

This implies that
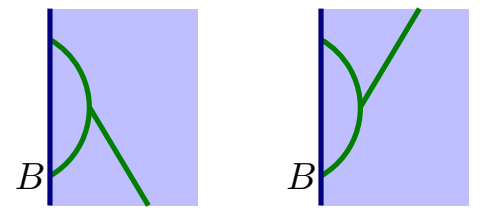

define $P$-module, respectively $P$-comodule structures on $B$, which are also inverse to each other and hence provide isomorphisms $B \otimes P \cong P$.

\section{A.4 P-adjunction}

In this appendix we show that the adjunction of IR defects is lifted to the UV by the following formulas

$$
\begin{aligned}
& D^{\dagger P}=P \otimes D^{\dagger} \otimes P \\
& { }^{\dagger} D=P \otimes{ }^{\dagger} D \otimes P .
\end{aligned}
$$

where $P$ is the corresponding projection defect, and $D$ is a defect in the UV theory representing an IR defect. We will only consider the first equation and will furthermore restrict to the case that $P$ is unital. The arguments for the second equation and the counital case are similar. The IR right adjoints have to satisfy the following Zorro move identities
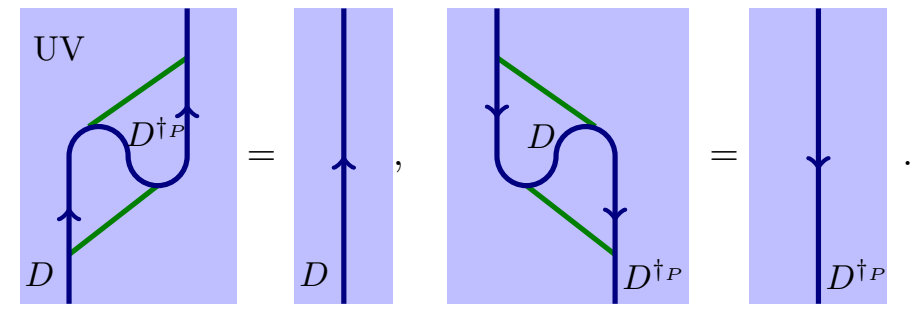

These are satisfied for $D^{\dagger P}$ above when we choose the following natural (co-)evaluation maps
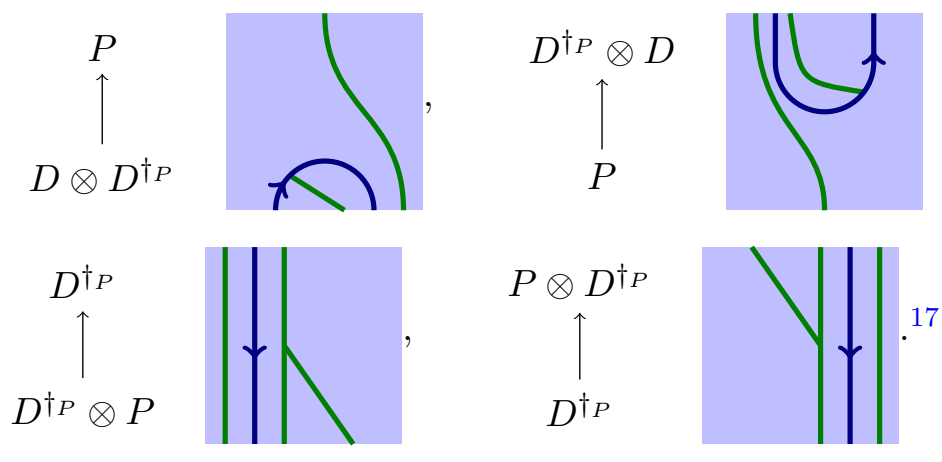
Namely,
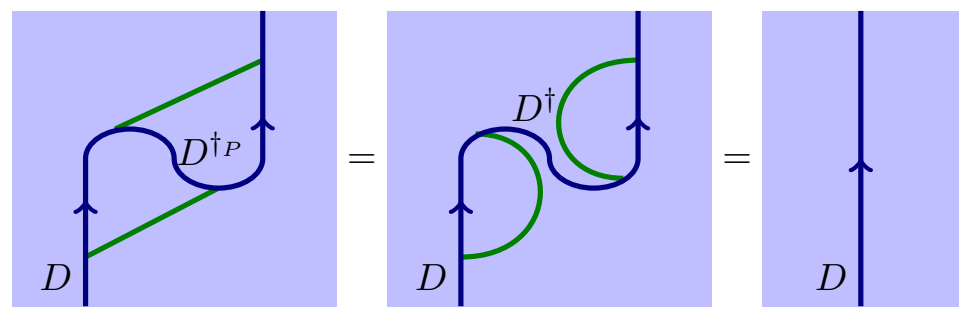

and
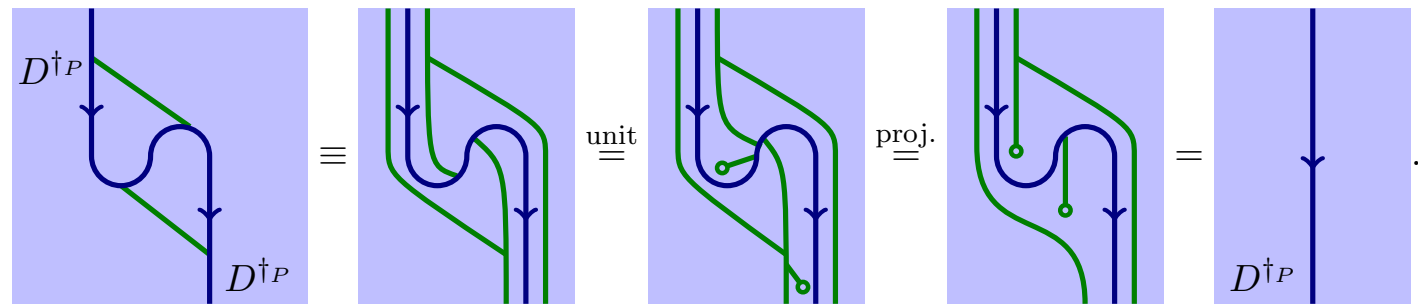

using unit condition, Zorro-move and the definition of $D^{\dagger P}$ in the last step. For counital $P$ and left-adjoints the above diagrams have to be flipped appropriately.

The defect $P$ is a $P$-module and a $P$-comodule, so it can be regarded as an IR defect. As such, it should be selfadjoint, and, using the above notion of IR adjunction one finds that this is indeed the case: $P^{\dagger} P \cong P \cong{ }^{\dagger} P$. If for instance $P$ is unital, the two maps
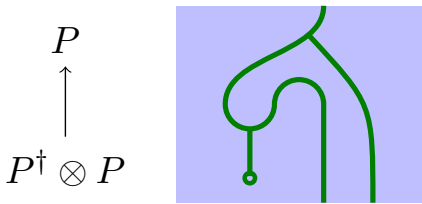

and
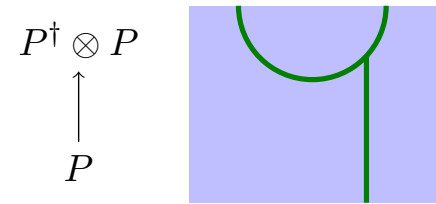

are inverse to each other and hence provide isomorphisms $P \cong P^{\dagger} \otimes P$. The projection property of $P, P \otimes P \cong P$ implies that $P^{\dagger} P=P \otimes P^{\dagger} \otimes P \cong P$. The argument for counital $P$ is analogous.

\section{A.5 Projections with unit and counit}

If a projection defect has a unit as well as a counit, it is automatically self-adjoint as there are natural (co)evaluation maps:
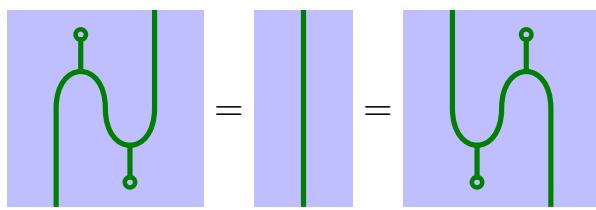

\footnotetext{
${ }^{17}$ The lower maps follow as natural generalizations from the generalized orbifold procedure [5, Prop. 4.7]. Namely,
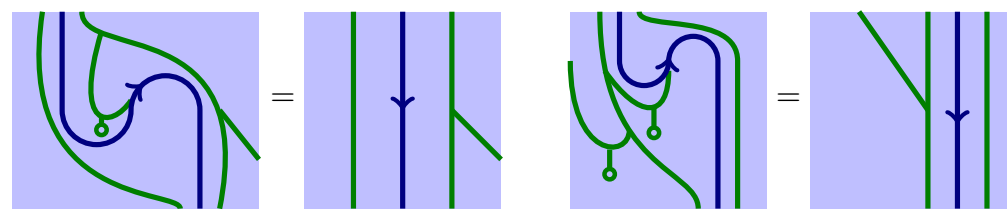
The equalities follow from the two Frobenius and (co)unit properties. If $P$ has a unit, any $P$-module automatically carries a $P$-comodule structure. Vice versa, any $P$-comodule is automatically a $P$-module in case $P$ has a counit. If $P$ has both, a unit and a counit, these two constructions are compatible: Starting with a $P$-module, a $P$-comodule structure is induced which in turn induces a $P$-module structure. This $P$-module structure is identical to the original one:

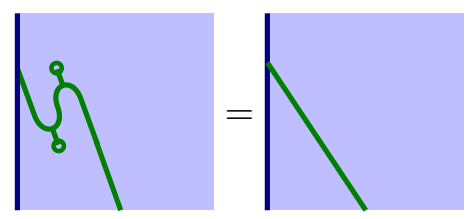

As discussed in section 3.5, all projection defects $P$ factorize into RG type defects $P \cong R^{\dagger} \otimes R$. For example, unitary $P$ factorize as

$$
\begin{aligned}
& \left(P_{\mathrm{IR} \mid \mathrm{UV}}\right)^{\dagger}=P_{\mathrm{UV} \mid \mathrm{IR}} \\
& { }^{\dagger}\left(P_{\mathrm{IR} \mid \mathrm{UV}}\right)=\left({ }^{\dagger} P\right)_{\mathrm{UV} \mid \mathrm{IR}} .
\end{aligned}
$$

For selfadjoint projection defects $P$, the respective RG type defects $R$ then satisfy ${ }^{\dagger} R \cong R^{\dagger}$.

\section{A.6 Equivalence of (co)multiplication and Frobenius properties for projec- tions}

Here, we show the equivalence of associativity, coassociativity and the two Frobenius properties for projection defects and how they follow from the existence of a (co)unit. The identities in question are:

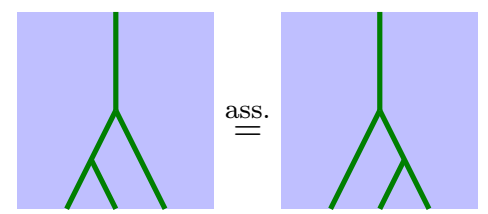

and
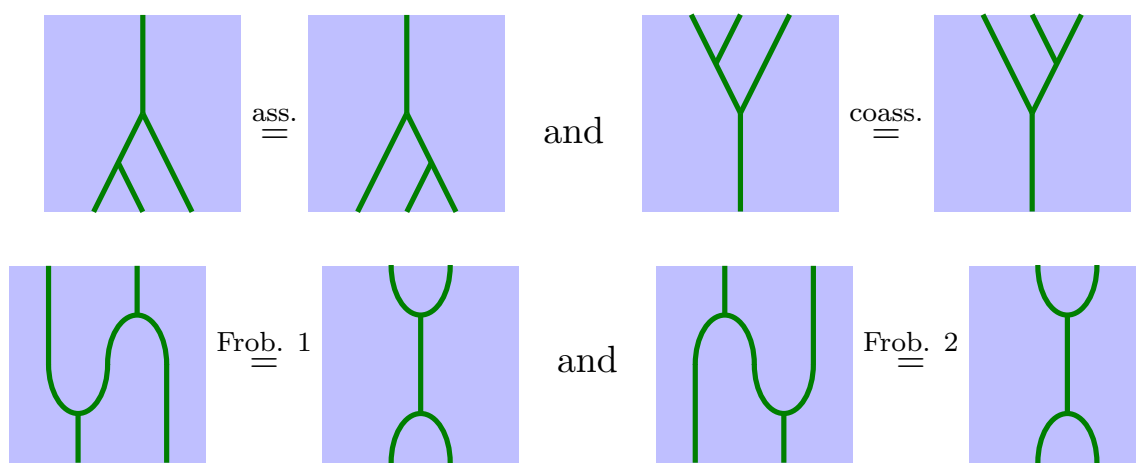

and

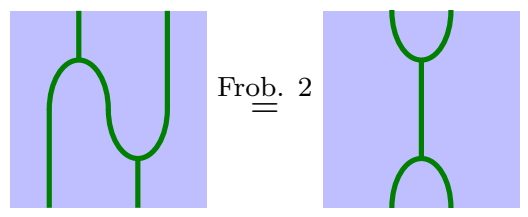

Equivalence is shown by the following chain of implications

$$
\text { associativity } \Rightarrow \text { Frob. } 2 \Rightarrow \text { coassociativity } \Rightarrow \text { Frob. } 1 \Rightarrow \text { associativity. }
$$

associativity $\Rightarrow$ Frob. 2:

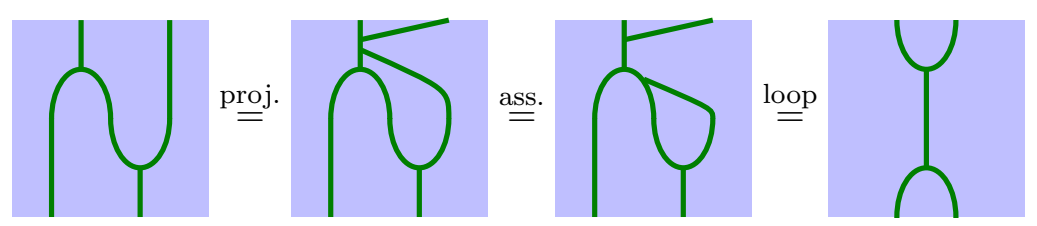


Frob. $2 \Rightarrow$ coassociativity:

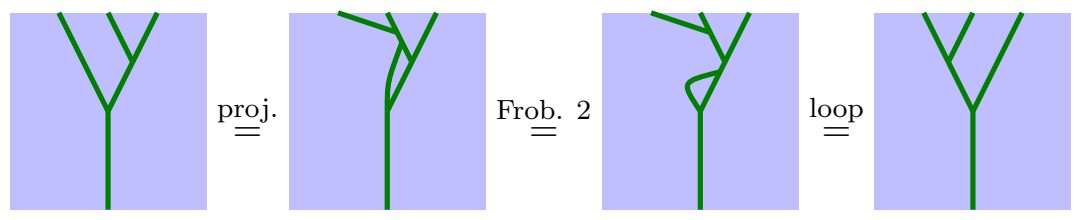

coassociativity $\Rightarrow$ Frob. 1:
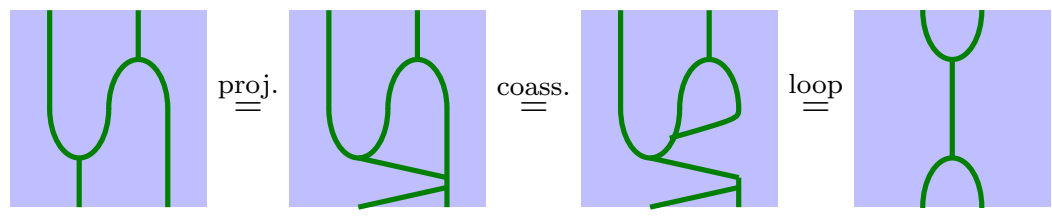

Frob. $1 \Rightarrow$ associativity:
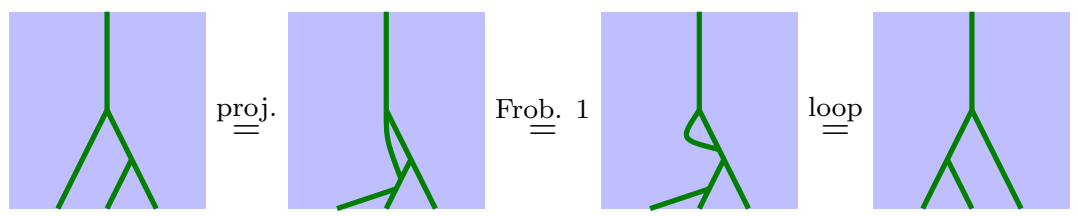

Next, we show how the existence of a unit for a projection defect implies coassociativity:

unit $\Rightarrow$ coassociativity:
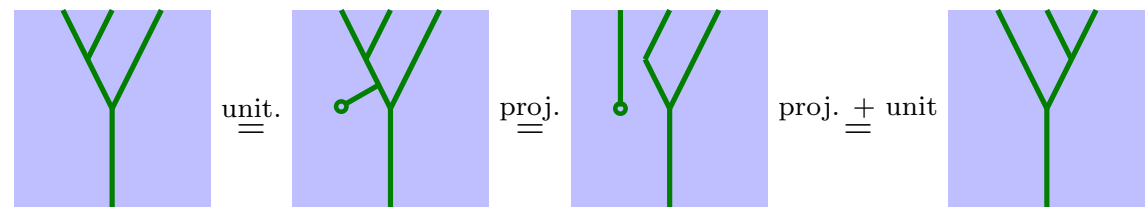

In the last step we applied the projection property to the left and the lower defect. Turning these diagrams upside down shows how associativity follows from the existence of a counit.

\section{A.7 Adjoints of induced RG defects}

Right and left adjoints of the induced RG defect $P_{\mathrm{IR} \mid \mathrm{UV}}$ must satisfy the Zorro move identities

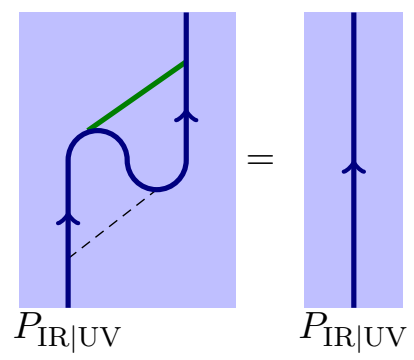

and

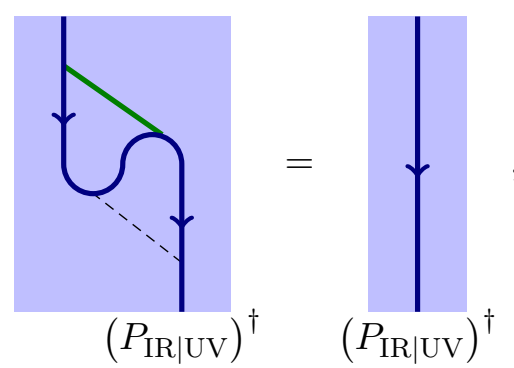



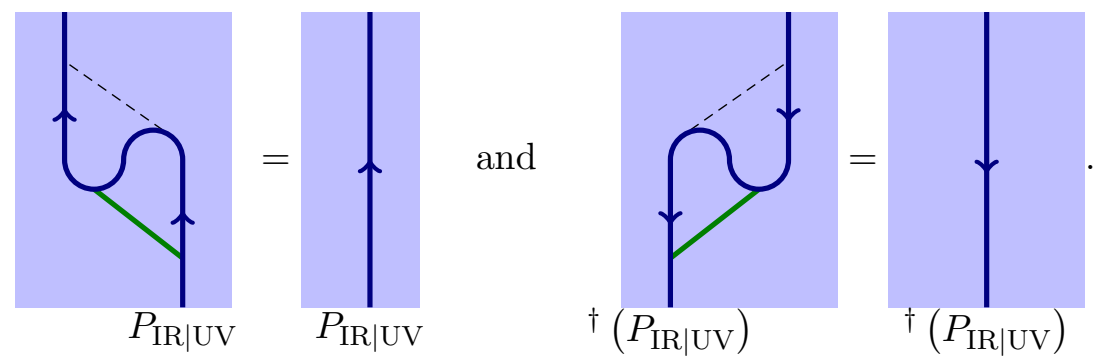

We will discuss the case that $P$ has a unit. The counital case can be treated analogously. Indeed, it is easy to see that

$$
\left(P_{\mathrm{IR} \mid \mathrm{UV}}\right)^{\dagger}=P_{\mathrm{UV} \mid \mathrm{IR}}
$$

i.e. $P$ regarded as defect from IR to UV is the right adjoint of $P$ regarded as a defect from UV to IR. The evaluation map is just given by the algebra $P \otimes P \rightarrow P$ and the coevaluation map is induced by the unit $I_{\mathrm{UV}} \rightarrow P \rightarrow P \otimes P$. (The Zorro identities immediately follow from associativity and the unit condition.) It is slightly more involved to see that the left adjoint is given by

$$
\dagger\left(P_{\mathrm{IR} \mid \mathrm{UV}}\right)=\left({ }^{\dagger} P\right)_{\mathrm{UV} \mid \mathrm{IR}},
$$

the left adjoint of $P$ regarded as a defect from the IR to the UV theory. Evaluation and coevaluation are given by the maps
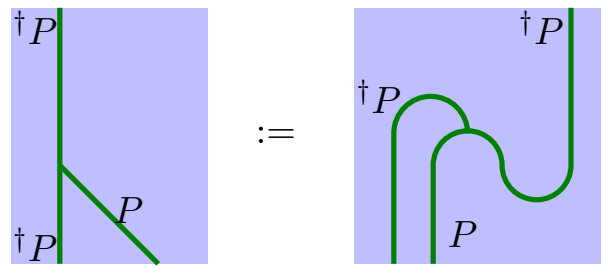

and
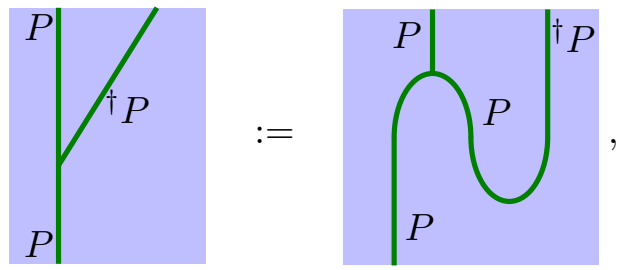

which define the right $P$-module structure of ${ }^{\dagger} P$ and the right ${ }^{\dagger} P$-comodule structure of $P$, respectively. The first Zorro identity then follows from the UV Zorro move and loop omission, while the second one additionally requires associativity and the unit property.

\section{B Generalized orbifold theories}

The generalized orbifold procedure $[4,5,7,8]$ is a method to produce a new theory out of a given 2d TQFT $T$ by inserting networks of an endo-defect $A: T \rightarrow T$ into its correlation functions. These modified correlation functions are well-defined if the defect $A$ satisfies the following special properties. It has to come with (co)multiplication and (co)unit fields

$$
\oint A \otimes A \rightarrow A, \quad\} A \rightarrow A \otimes A, \quad\lceil A \rightarrow I, \quad \text { II } \rightarrow A
$$

which make $A$ into a separable Frobenius algebra, i.e. it obeys the (co)associativity and (co)unit conditions

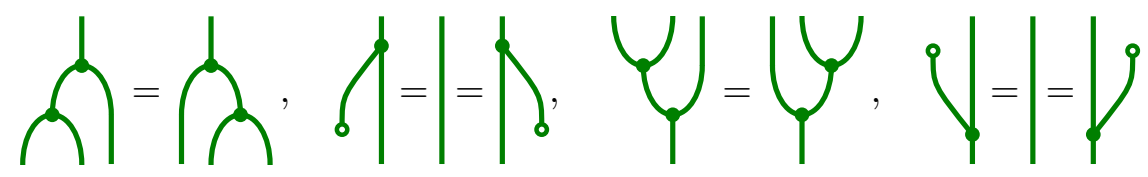


as well as the Frobenius and loop-omission properties:

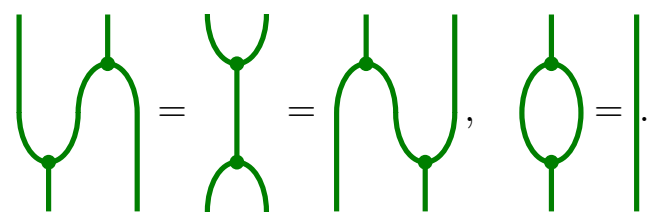

The respective orbifold theory is denoted by $(T, A)$. An obvious example for a defect satsifying the above conditions is the identity defect $A=I$ in any TQFT. Orbifolding by $I$ of course just gives back the original theory, $(T, I) \cong T$. In the following we will briefly outline how objects in the orbifold $(T, A)$ are defined in terms of objects in $A$.

For any two TQFTs $T$ and $T^{\prime}$ with defects $A$ and $A^{\prime}$ as above an $A$ - $A^{\prime}$-bimodule $D$ is a defect $D: T^{\prime} \rightarrow T$ with junctions $A \otimes D \rightarrow D, D \otimes A^{\prime} \rightarrow D$ such that
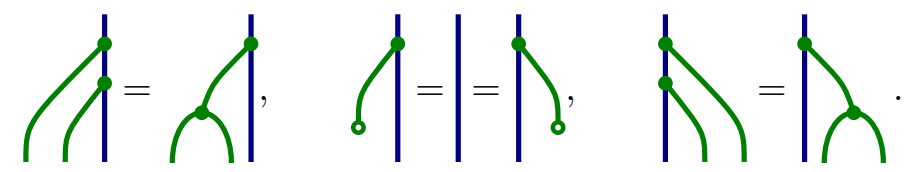

For two such bimodules $D$ and $\tilde{D}, \operatorname{Hom}_{A, A^{\prime}}(D, \tilde{D})$ denotes the space of all defect changing fields $D \rightarrow \tilde{D}$ commuting with the bimodule structure, i.e.

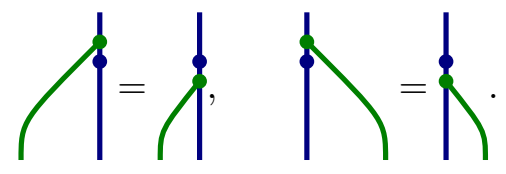

Via the unit, such modules are automatically also comodules, cf. [5, eqn. (4.1)]:

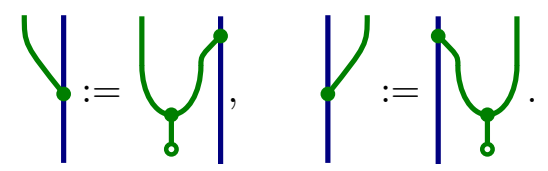

With these notations at hand, one can now represent objects of the generalized orbifold theory (T, A) in terms of objects of $T$ as follows:

i) Its invisible defect is $A$.

ii) Its bulk Hilbert space is $\operatorname{Hom}_{A, A}(A, A)$, the space of $A$ - $A$-bimodule endomorphisms of $A$.

iii) Boundary conditions $B$ of $(T, A)$ are those boundary conditions $B$ of $T$ carrying an appropriate $A$-module structure.

iv) The space of boundary condition changing fields between boundary conditions $B$ and $\tilde{B}$ is given by $\operatorname{Hom}_{A}(B, \tilde{B})$, the space of $A$-module morphisms from $B$ to $\tilde{B}$.

v) Defects $D$ from $\left(T^{\prime}, A^{\prime}\right)$ to $(T, A)$ are $A$ - $A^{\prime}$-bimodules.

vi) The space of defect changing fields from defects $D$ to $\tilde{D}$ is given by $\operatorname{Hom}_{A, A^{\prime}}(D, \tilde{D})$, the space of $A-A^{\prime}$-bimodule morphisms from $D$ to $\tilde{D}$. 
vii) The fusion product $D \otimes_{A} \tilde{D}$ in the orbifold theory $(T, A)$ of two defects $D$ and $\tilde{D}$ is given by the image of the fusion $D \otimes \tilde{D}$ in the unorbifolded theory $T$ under

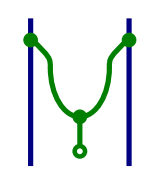

viii) The adjoints of defects $D$ in the orbifold theory are defined in the following way in terms of the adjoints in the unorbifolded theory. The $A$-actions on any defect $D$ in $(T, A)$ induce actions on its non-orbifold adjoints ${ }^{\dagger} D$ and $D^{\dagger}$ :

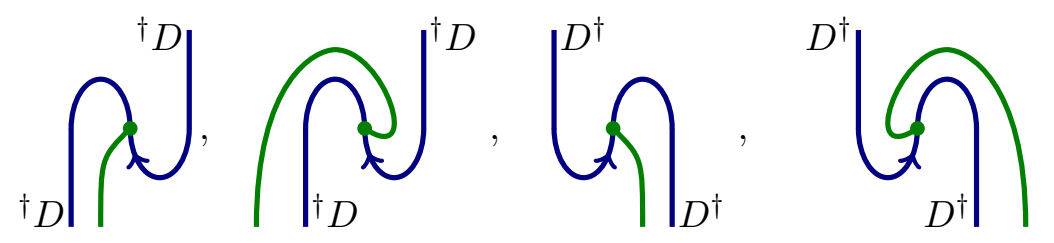

The action of an algebra $A$ on any module can be twisted by an algebra automorphism $\alpha: A \rightarrow A$. So for any defect $D$ in the orbifold theory, one can define twisted defects ${ }_{\alpha}(D)$ and $(D)_{\alpha}$ by twisting the left, respectively right $A$-action:

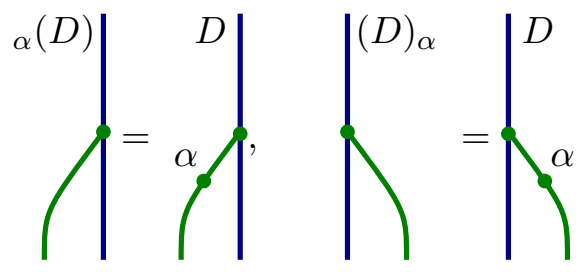

Left and right adjoints in the orbifold theory can be obtained by twisting the respective adjoints in the unorbifolded theory by the Nakayama automorphism

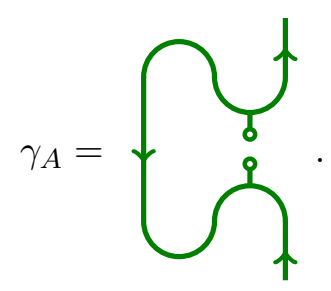

More precisely, the left and right adjoints in the orbifold theory ${ }^{18}$ are given by $[5$, Prop. 4.7]

$$
{ }^{*} D=\gamma_{A}^{-1}\left({ }^{\dagger} D\right), \quad D^{*}=\left(D^{\dagger}\right)_{\gamma_{A^{\prime}}} .
$$

For ${ }^{*} D$, the (co)evaluation maps are given by

$$
\mathrm{ev}_{D}=\bigcap_{D} \bigcap_{D} \circ \xi, \quad \operatorname{coev}_{D}=\vartheta \circ{ }^{D}
$$

with the inclusion and projections maps $\xi:{ }^{*} D \otimes_{A} D \rightarrow{ }^{*} D \otimes D$ and $\vartheta: D \otimes{ }^{*} D \rightarrow$ $D \otimes_{A}{ }^{*} D$. There are similar formulas for the (co)evaluation maps for $D^{*}$.

\footnotetext{
${ }^{18}$ In this appendix we denote adjunction in the orbifold by ${ }^{*}$, to distinguish it from the adjunction ' ${ }^{\prime}$, in the unorbidolded theory.
} 


\section{Orbifold minimal models as generalized orbifolds}

Here, we construct the Landau-Ginzburg orbifolds $\mathcal{M}_{d} / \mathbb{Z}_{d}$ as generalized orbifolds following [5, Chapter 7]. To distinguish objects in the orbifold from objects in the unorbifolded theory, we adopt the following notation, which is different from the one used in the main text: the identity defect in $\mathcal{M}_{d}$ is denoted by $I$, whereas the identity defect in the orbifold theory is represented by the orbifold defect $A$. Also, adjunction in the orbifold is denoted by ${ }^{*} *$ ' to distinguish it from adjunction ' $\dagger$ ' in the unorbifolded theory. This notation is only used in this appendix. In section 4 of the main text, we do not explicitly refer to the orbifold construction and therefore do not need this distinction. There, $I$ denotes the identity defect and ' $\dagger$ ' the adjunction in the orbifold theory $\mathcal{M}_{d} / \mathbb{Z}_{d}$.

\section{C.1 Orbifold identity defect}

The models $\mathcal{M}_{d} / \mathbb{Z}_{d}$ are standard orbifolds. In this case the defect $A$ is given by the direct sum of the defects implementing the respective actions of all the symmetries in the orbifold group: $A=\oplus_{g \in \mathbb{Z}_{d}}(g I)$. The symmetry defects ${ }_{g} I$ can be represented by the rank-one matrix factorizations $\left(\eta=e^{2 \pi i / d}\right)$

$$
{ }_{g} I: \mathbb{C}[Z, X] e_{d+[g]} \frac{\eta^{g} Z-X}{\frac{Z^{d}-X^{d}}{\eta^{g} Z-X}} \mathbb{C}[Z, X] e_{[g]} .
$$

where $[g]$ denotes the representative of $g \in \mathbb{Z}_{d}$ in $\{0, \ldots, d-1\}$, and the $e_{a}, a \in\{0, \ldots, 2 d-$ $1\}$ are the generators of the respective rank-one free modules $\left({ }_{g} I\right)_{0,1} \cdot{ }_{g} I$ is the right twist of the identity defect $I$ in $\mathcal{M}_{d}$ by $g \in \mathbb{Z}_{d}$.

Since $A$ is the direct sum of the ${ }_{g} I$, the modules $A_{0}$ and $A_{1}$ are rank- $d$ free modules generated by $e_{0}, \ldots, e_{d-1}$ and $e_{d}, \ldots, e_{2 d-1}$, respectively. In the basis $\left(e_{a}\right)$, the differential of the matrix factorization $A$ takes the form

$$
\left(\mathrm{d}_{A}\right)_{a b}=\delta_{a, b-d}\left(\eta^{a} Z-X\right)+\delta_{a-d, b} \sum_{l=1}^{d} \eta^{-l \cdot a} Z^{d-l} X^{l-1}
$$

for $a, b=0, \ldots, 2 d-1$.

The following maps give $A$ a separable Frobenius structure [5, Prop. 7.1]:

1. The unit $I={ }_{0} I \hookrightarrow A$ is given by the obvious inclusion while the counit is the projection multiplied by $\mathrm{d}$

$$
A \rightarrow I, \quad e_{i} \mapsto d \cdot\left\{\begin{array}{l}
e_{i}, i \in\{0, d\} \\
0, \text { otherwise }
\end{array}\right.
$$

2. Multiplication and comultiplication
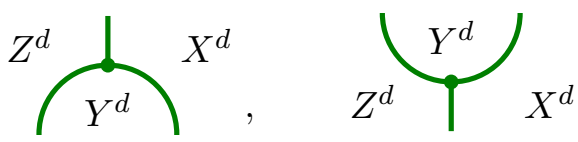
are given by

$$
\begin{aligned}
A \otimes A & \rightarrow A \\
e_{[g]} \otimes Y^{q} e_{[h]} & \mapsto\left(\eta^{g} Z\right)^{q} e_{[g+h]} \\
e_{[g]} \otimes Y^{q} e_{d+[h]} & \mapsto\left(\eta^{g} Z\right)^{q} e_{d+[g+h]} \\
e_{[g]+d} \otimes Y^{q} e_{[h]} & \mapsto 0 \\
e_{[g]+d} \otimes Y^{q} e_{[h]+d} & \mapsto 0
\end{aligned}
$$

and

$$
\begin{aligned}
\Delta: A & \rightarrow A \otimes A \\
e_{[g]} & \mapsto \frac{1}{d} \sum_{h \in \mathbb{Z}_{d}}\left[e_{[g-h]} \otimes e_{[h]}+e_{d+[g-h]} \otimes\left\{\partial^{Z, Y} \frac{Z^{d}-X^{d}}{\eta^{h} Z-X}\right\}^{Z \rightarrow \eta^{g-h} Z} e_{d+[h]}\right] \\
e_{d+[g]} & \mapsto \frac{1}{d} \sum_{h \in \mathbb{Z}_{d}}\left[e_{[g-h]} \otimes e_{d+[h]}+\eta^{h} e_{d+[g-h]} \otimes e_{[h]}\right],
\end{aligned}
$$

where $g, h \in \mathbb{Z}_{d}$ and $q \in \mathbb{N}$. Moreover, $\partial^{Z, Y} Z^{i}=\frac{Z^{i}-Y^{i}}{Z-Y}$ and $\{\ldots\}^{Z \rightarrow \eta^{g-h} Z}$ means that all instances of $Z$ within the brakets have to be replaced by $\eta^{g-h} Z$ after performing all operations. These formulas can be obtained from the natural junctions of symmetry defects ${ }_{h} I$ with the identity defect $I={ }_{0} I$. The calculation for the comultiplication is sketched in appendix C.7.1 below.

In appendix C.6 below, we will reexpress the orbifold identity defect $A$ using an equivariant basis.

\section{C.2 Nakayama automorphism}

The Nakayama automorphism (cf. appendix B) takes the form [8, Example 3.1]

$$
\gamma_{A}=\sum_{g \in \mathbb{Z}_{d}} \operatorname{det}(g) \cdot 1_{g} I
$$

where $\operatorname{det}(g)$ denotes the matrix representing the action of $g$ on the chiral fields of the model to the right of $A$. In our case, $g$ acts on $X$ by multiplication with $\eta^{g}$ and hence $\gamma_{A}$ reduces to

$$
\begin{aligned}
\gamma_{A}: A & \rightarrow A \\
e_{a} & \mapsto \eta^{a} e_{a} .
\end{aligned}
$$

A Frobenius algebra $B$ is symmetric iff $\gamma_{B}=\operatorname{id}_{B}[5,40]$. Since $\gamma_{A} \neq \operatorname{id}_{A}, A$ is not symmetric. Therefore, left and right adjoints of defects in the orbifold theory (see equation (B.1)) generally differ

$$
\left(D^{\dagger}\right)_{\gamma_{A^{\prime}}}=D^{*} \not{ }^{*} D={ }_{\gamma_{A}^{-1}}\left({ }^{\dagger} D\right)
$$

This means that we do not have a general prescription of how to close defect loops in $\mathcal{M}_{d} / \mathbb{Z}_{d}$. However, loops of RG defects can be closed with an explicit natural morphism. 


\section{C.3 Bulk space}

The $(c, c)$-bulk space of the orbifold $\mathcal{M}_{d} / \mathbb{Z}_{d}$ contains only the identity id : $A \rightarrow A$. However, in the unorbifolded theory the defect $A$ carries additional fields - one for each $g \neq 0$ :

$$
\begin{aligned}
\psi_{g}: A & \rightarrow A \\
e_{[h]} & \mapsto \frac{Z^{d}-X^{d}}{\left(\eta^{i} Z-X\right)\left(\eta^{h+g} Z-X\right)} e_{d+[g+h]} \\
e_{d+[h]} & \mapsto e_{[g+h]}
\end{aligned}
$$

These correspond to the twist fields in the orbifold theory.

\section{C.4 Defects and their adjoints}

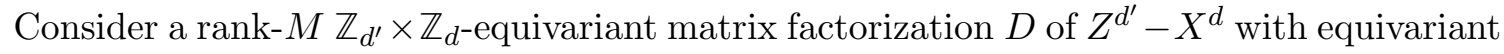

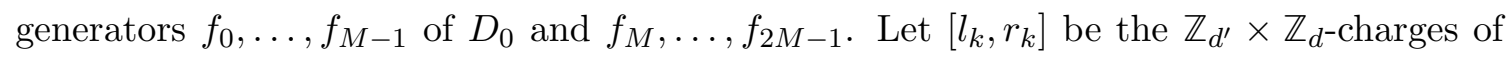
$f_{k}$. As discussed in the second part of C.7, these charges determine the $A$-action on $D$. Denoting the chiral fields as in

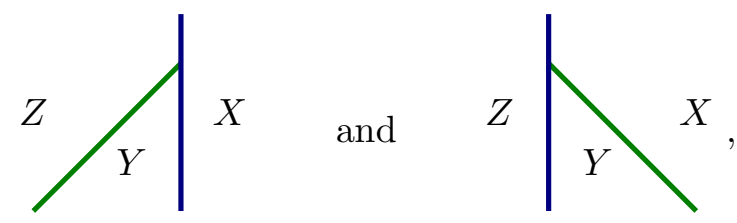

one obtains

$$
\begin{aligned}
A \otimes D & \rightarrow D \\
e_{a} \otimes Y^{p} f_{k} & \mapsto \delta_{\left|e_{a}\right|, 0} \cdot\left(\epsilon^{a} Z\right)^{p} \cdot \epsilon^{a \cdot l_{k}} \cdot f_{k} .
\end{aligned}
$$

and

$$
\begin{aligned}
D \otimes A & \rightarrow D \\
f_{k} \otimes Y^{p} e_{a} & \mapsto \delta_{\left|e_{a}\right|, 0} \cdot f_{k} \cdot \eta^{-a \cdot r_{k}}\left(\eta^{-a} X\right)^{p}
\end{aligned}
$$

where $\epsilon=e^{\frac{2 \pi i}{d^{\prime}}}$ and $\eta=e^{\frac{2 \pi i}{d}}$ are elementary $d^{\prime}$ th, respectively $d$ th roots of unity.

In appendix C.6 below, we will define equivariant generators for $A$ itself, and will reexpress the $A$-action on $D$ in terms of these generators.

The adjoints in the orbifold theory are given by ${ }^{*} D={ }_{\gamma_{A}^{-1}}\left({ }^{\dagger} D\right)$ and $D^{*}=\left(D^{\dagger}\right)_{\gamma_{A^{\prime}}}$, see equation (B.1). Here $D^{\dagger} \cong D^{\vee}[1] \cong{ }^{\dagger} D$ denotes adjunction in the unorbifolded models, cf. section 4.1. An explicit calculation carried out in the last part of appendix C.7 determines the induced $A$-action on $D^{\dagger}$, cf. equation (C.10). From this, one can read off the $\mathbb{Z}_{d} \times \mathbb{Z}_{d^{\prime}}$ charges of the equivariant generators $f_{k}^{\dagger}$ and ${ }^{\dagger} f_{k}$ of $D^{\dagger}$ and ${ }^{\dagger} D$ to be

$$
\left[-r_{k+M}+1,-l_{k+M}+1\right]
$$

Here, we have extended the range of indices of the charges $r$ and $l$ to $\mathbb{Z}$ by identification modulo $2 M$, i.e. $r_{i+2 M z}=r_{i}$ and $l_{i+2 M z}=l_{i}$ for $i \in\{0, \ldots, 2 M-1\}$ and $z \in \mathbb{Z}$. 
Twisting by the Nakayama automorphism one then obtains the charges of the generators $f_{k}^{*}$ and ${ }^{*} f_{k}$ of the matrix factorizations describing the orbifold adjoints $D^{*}$ and ${ }^{*} D$. They are given by

$$
\text { and } \quad \begin{array}{ll} 
& {\left[-r_{k+M},-l_{k+M}+1\right]} \\
& {\left[-r_{k+M}+1,-l_{k+M}\right],}
\end{array}
$$

respectively. By construction, ${ }^{*} D$ and $D^{*}$ obey the Zorro moves whose building blocks are provided in C.8.

\section{C.5 Left boundary conditions and their adjoints}

As special case of defects, a left boundary condition in $\mathcal{M}_{d} / \mathbb{Z}_{d}$ is a $\mathbb{Z}_{d}$-equivariant matrix factorization $B$ of $-X^{d}$. Using the same notation as in appendix C.4, we denote the generators of the modules $B_{0}$ and $B_{1}$ by $f_{k}$ and their $\mathbb{Z}_{d}$-charges by $\left[r_{k}\right]$ which as in the general case determine the $A$-action on $B$. The induced charges on the right and left adjoint generators $f_{k}^{\dagger}$ of $B^{\dagger}$ and ${ }^{\dagger} f_{k}$ of ${ }^{\dagger} B$ are $\left[-r_{k+M}+1\right]$ and $\left[-r_{k}+1\right]$, respectively. Using $B^{*}=B^{\dagger}$ and ${ }^{*} B={ }_{\gamma^{-1}}\left({ }^{\dagger} B\right)$, the charges of the adjoint generators $f_{k}^{*}$ and ${ }^{*} f_{k}$ become

$$
\begin{aligned}
f_{k}^{*} & :\left[-r_{k+M}+1\right] \\
{ }^{*} f_{k} & :\left[-r_{k}\right]
\end{aligned}
$$

The explicit expressions of the relevant (co-)evaluation maps for defects as well as boundaries are given in appendix C.8.

\section{C.6 Equivariant generators of the orbifold identity defect}

One can define equivariant generators of the orbifold identity matrix factorization $A$ (cf. section C.1) by

$$
e_{b}^{\prime}=\frac{1}{d} \sum_{c} \delta_{\left|e_{b}\right|,\left|e_{c}\right|} \eta^{-\left(b+\left|e_{b}\right|\right) c} e_{c},
$$

where the original generators $e_{c}$ are expressed in terms of the equivariant ones as

$$
e_{c}=\sum_{b} \delta_{\left|e_{b}\right|,\left|e_{c}\right|} \eta^{c\left(b+\left|e_{c}\right|\right)} e_{b}^{\prime}
$$

In this basis, the matrix factorization $A$ takes the equivariant form

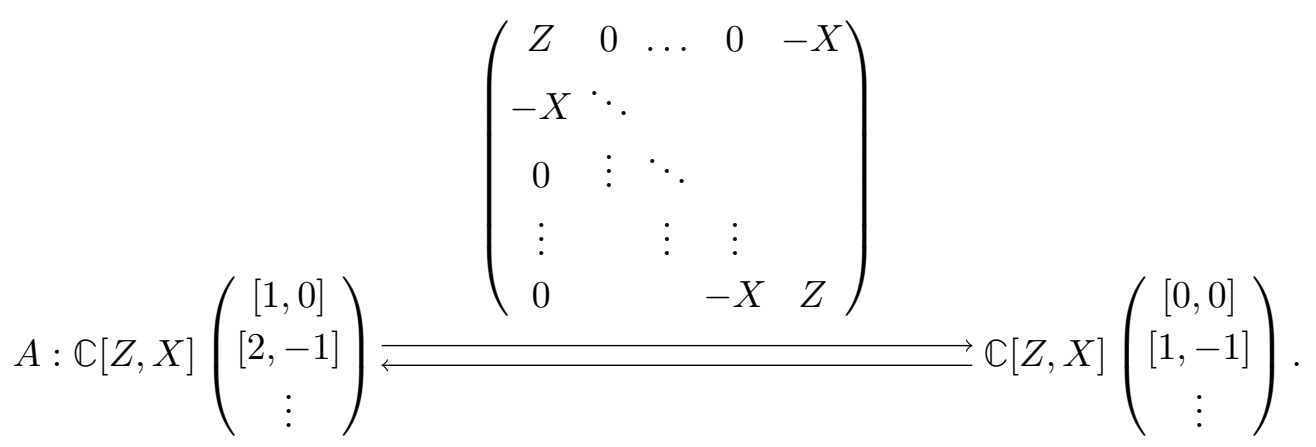

(This is the form used in [1].) The $A$-action on equivariant matrix factorizations determined in appendix C.7 and used in appendix C.4 simplifies in this basis. 
Consider a $\mathbb{Z}_{d} \times \mathbb{Z}_{d^{-}}$-equivariant matrix factorization $D$ of $Z^{d}-X^{d}$. Let $f_{0}, \ldots, f_{M-1}$ and $f_{M}, \ldots, f_{2 M-1}$ be $\mathbb{Z}_{d} \times \mathbb{Z}_{d}$-equivariant generators of $D_{0}$ and $D_{1}$, respectively. Denote the $\mathbb{Z}_{d} \times \mathbb{Z}_{d}$-charges of $f_{k}$ by $\left[l_{k}, r_{k}\right]$. In terms of the equivariant generators $e_{i}^{\prime}$ of $A$, the $A$-action

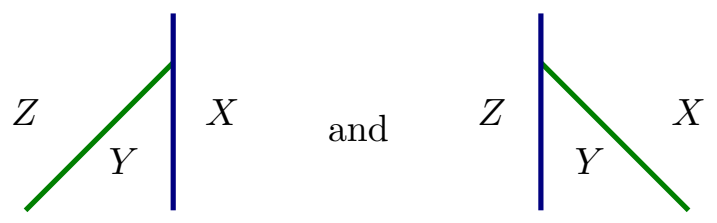

becomes

$$
\begin{array}{ll}
A \otimes D \rightarrow D, & e_{a}^{\prime} \otimes Y^{p} f_{k} \mapsto \delta_{a,\left[p+l_{k}\right]} Z^{p} f_{k} \\
D \otimes A \rightarrow D, & f_{k} \otimes Y^{p} e_{a}^{\prime} \mapsto \delta_{a,\left[-r_{k}-p\right]} f_{k} X^{p} .
\end{array}
$$

\section{C.7 Important calculations}

In this appendix we sketch some calculations used in the main text and the previous sections of this appendix.

\section{C.7.1 Comultiplication of identity defect $A$}

Following [5, 12], we define $\lambda_{h I}^{-1}:{ }_{h} I \rightarrow I \otimes{ }_{h} I$ to be the natural junction of the identity defect with the symmetry defect ${ }_{h} I$. It is given by

$$
\begin{aligned}
e_{[h]} & \mapsto 1 \otimes e_{[h]}+\theta \otimes\left\{\partial^{Z, Y} \frac{Z^{d}-X^{d}}{\eta^{h} Z-X}\right\} e_{d+[h]} \\
e_{d+[h]} & \mapsto 1 \otimes e_{d+[h]}+\eta^{h} \theta \otimes e_{[h]} .
\end{aligned}
$$

Here $X$ and $Z$ are the chiral fields of the models to the right, respectively left of the defects, and $Y$ is the chiral field of the model sandwiched between the defects $I$ and ${ }_{h} I$. Twisting by $g$ from the left (i.e. fusion by ${ }_{g} I$ from the left) one obtains junction fields $\Delta_{g, h}:={ }_{g}\left(\lambda_{h}^{-1}\right):{ }_{g+h} I \rightarrow{ }_{g} I \otimes{ }_{h} I:$

$$
\begin{aligned}
e_{[g+h]} & \mapsto e_{[g]} \otimes e_{[h]}+e_{d+[g]} \otimes\left\{\partial^{Z, Y} \frac{Z^{d}-X^{d}}{\eta^{h} Z-X}\right\}^{Z \rightarrow \eta^{g} Z} e_{d+[h]} \\
e_{d+[g+h]} & \mapsto e_{[g]} \otimes e_{d+[h]}+\eta^{h} e_{d+[g]} \otimes e_{[h]} .
\end{aligned}
$$

(Here, the notation $\{\ldots\}^{Z \rightarrow \eta^{g} Z}$ means that all instances of $Z$ in the brackets have to be replaced by $\eta^{g} Z$ after performing all calculations.)

Summing up all the $\Delta_{g, h}$ yields the comultiplication

$$
\begin{aligned}
\Delta=\frac{1}{d} \sum_{g, h} \Delta_{g, h}: A & \rightarrow A \otimes A \\
e_{[g]} & \mapsto \frac{1}{d} \sum_{h \in \mathbb{Z}_{d}} \Delta_{g-h, h}\left(e_{[g]}\right) \\
e_{d+[g]} & \mapsto \frac{1}{d} \sum_{h \in \mathbb{Z}_{d}} \Delta_{g-h, h}\left(e_{d+[g]}\right)
\end{aligned}
$$

of the identity defect $A$ in the orbifold. It is spelled out completely in equation (C.2). 
$\boldsymbol{A}$ actions on equivariant defect. According to [5, Section 7.1], the data of a $G \times H$ equivariant defect is encoded in its left and right fusion with the symmetry defects $A_{G}$ and $A_{H}$. Namely, it is described by a matrix factorization together with isomorphisms

- $\varphi_{g}:{ }_{g} D \rightarrow D$ such that $\varphi_{e}=\operatorname{id}_{D}$ and $\varphi_{g_{1}} \circ{ }_{g_{1}}\left(\varphi_{g_{2}}\right)=\varphi_{g_{1}+g_{2}}$ and

- $\phi_{h}: D_{h} \rightarrow D$ such that $\phi_{e}=\operatorname{id}_{D}$ and $\phi_{h_{1}} \circ\left(\phi_{h_{2}}\right)_{h_{1}}=\phi_{h_{1}+h_{2}}$.

Here, one can think of ${ }_{g} D$ as the matrix factorization where all variables $Z_{i}$ to the left of $D$ have been replaced by $g\left(Z_{i}\right)$, see for example ${ }_{g} I$ in (C.1). Also, for some morphism $\alpha: D \rightarrow D^{\prime}$ of matrix factorizations, ${ }_{g}(\alpha)_{h}:{ }_{g} D_{h} \rightarrow{ }_{g} D^{\prime}{ }_{h}$ is the same morphism considered as a morphism between the respective twisted matrix factorizations. However, special attention has to be paid to morphisms including an identification of variables. For example, the left and right $I$-actions $\lambda_{D}: I \otimes D \rightarrow D$ and $\rho_{D}: D \otimes I \rightarrow D$ identify the middle variable with the one on the left or right, respectively. The identification of variables in the twisted versions $g\left(\lambda_{D}\right)$ and $\left(\rho_{D}\right)_{-h}$ must respect the twist.

Following the proof of Thm. 7.2 in [5], the above data determine the left $A_{G}$-action on $D$ :

$$
\sum_{g \in G}\left(A_{G} \otimes D \rightarrow{ }_{g} I \otimes D \stackrel{g^{\left(\lambda_{D}\right)}}{\longrightarrow}{ }_{g} D \stackrel{\varphi_{g}}{\longrightarrow} D\right) .
$$

The right action includes the canonical isomorphism ${ }_{h} I \rightarrow I_{-h}$ which we will comment on later:

$$
\sum_{h \in H}\left(D \otimes A_{H} \rightarrow D \otimes{ }_{h} I \rightarrow D \otimes I_{-h} \stackrel{\left(\rho_{D}\right)_{-h}}{\longrightarrow} D_{-h} \stackrel{\phi_{-h}}{\longrightarrow} D\right) .
$$

Turning to our example, set $G=\mathbb{Z}_{d^{\prime}}$ and $H=\mathbb{Z}_{d}$ and consider a $G \times H$-equivariant

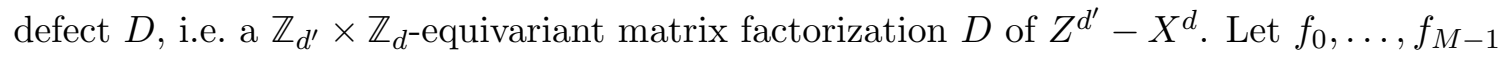
and $f_{M}, \ldots, f_{2 M-1}$ be equivariant generators of $D_{0}$, respectively $D_{1}$. Denote the $\mathbb{Z}_{d^{\prime}} \times \mathbb{Z}_{d^{-}}$ charges of $e_{k}$ by

$$
\left[l_{k}, r_{k}\right]
$$

In other words the action of $(g, h) \in \mathbb{Z}_{d^{\prime}} \times \mathbb{Z}_{d}$ is given by

$$
Z^{p} f_{k} X^{q} \mapsto\left(\epsilon^{g} Z\right)^{p} \cdot \epsilon^{g \cdot l_{k}} f_{k} \eta^{h \cdot r_{k}} \cdot\left(\eta^{h} X\right)^{q}
$$

where $\epsilon=e^{\frac{2 \pi i}{d^{\prime}}}, \eta=e^{\frac{2 \pi i}{d}}$.

We now reformulate this group action in terms of left and right $A$-actions. It is not hard to see that in our case the above isomorphisms are given by ${ }^{19}$

$$
\begin{aligned}
\varphi_{g}:{ }_{g} D & \rightarrow D & \phi_{h}: D_{h} & \rightarrow D \\
f_{k} & \rightarrow \epsilon^{g \cdot l_{k}} f_{k} & f_{k} & \rightarrow f_{k} \eta^{h \cdot r_{k} .}
\end{aligned}
$$

The explicit form of the left $A$-action on $D$ then turns out to be

$$
\begin{aligned}
A \otimes D & \rightarrow D \\
e_{a} \otimes Y^{p} f_{k} & \mapsto \delta_{\left|e_{a}\right|, 0} \cdot\left(\epsilon^{a} Z\right)^{p} \cdot \epsilon^{a \cdot l_{k}} \cdot f_{k}
\end{aligned}
$$

\footnotetext{
${ }^{19} \varphi_{g} \circ g\left(\varphi_{h}\right)=\varphi_{g+h}$ is trivial and $\mathrm{d}_{D} \circ \varphi_{g}=\varphi_{g} \circ{ }_{g}\left(\mathrm{~d}_{D}\right)$ amounts to $\mathrm{d}_{D}$ being a degree zero map, i.e. $e_{k}$ and $\mathrm{d}_{D}\left(e_{k}\right)$ carrying the same $\mathbb{Z}_{d^{\prime}} \times \mathbb{Z}_{d}$ charges.
} 
for the same choice of variables as in (C.3) $\left(|\cdot|\right.$ denotes the $\mathbb{Z}_{2}$-charge). This coincides with the expected action (C.5). The right $A$-action on the other hand takes the form

$$
\begin{aligned}
D \otimes A & \rightarrow D \\
f_{k} \otimes Y^{p} e_{a} & \mapsto \delta_{\left|e_{a}\right|, 0} \cdot f_{k} \cdot \eta^{-a \cdot r_{k}}\left(\eta^{-a} X\right)^{p}
\end{aligned}
$$

where we emphasize the crucial minus sign for the right charges which differs from the expected (C.5). It originates from the fact that the symmetry defect $A$ was defined as the direct sum of the left twisted identity morphisms which requires us to include the canonical isomorphism

$$
\begin{aligned}
{ }_{h} I & \rightarrow I_{-h} \\
e_{i} & \mapsto \eta^{h \cdot\left|e_{i}\right|} e_{i}, \quad i=0,1
\end{aligned}
$$

in the construction (C.4).

Left $\boldsymbol{A}$-action on right adjoint. As explained in appendix B item viii), adjoints of defects in the orbifold theory are induced by their non-orbifold counterpart. Here, given an equivarant matrix factorization $D$ of $Z^{d^{\prime}}-X^{d}$, we explicitely calculate the induced left $A$-action (i.e. the left charges, see previous calculation) on the non-orbifold adjoint $D^{\dagger}$. This leads to the charges of the right adjoint in the orbifold theory as $D^{*} \cong\left(D^{\dagger}\right)_{\gamma}$.

Let $f_{0}, \ldots, f_{M-1}$ be equivariant generators of $D_{0}$ and $f_{M}, \ldots, f_{2 M-1}$ equivariant gen-

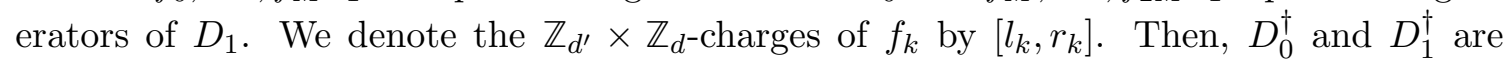
generated by $f_{0}^{\dagger}, \ldots, f_{M-1}^{\dagger}$ and $f_{M}^{\dagger}, \ldots, f_{2 M-1}^{\dagger}$ respectively, where $f_{i}^{\dagger}=f_{i+M}^{\vee}$ for $i<M$ and $f_{i}^{\dagger}=f_{i-M}^{\vee}$ for $i \geq M$. (' ${ }^{\vee}$, denotes the dual.)

From the explicit expressions of the (co-)evaluation maps [12] we obtain

$A \otimes D^{\dagger} \rightarrow D^{\dagger}$

$$
\begin{aligned}
& e_{a} \otimes f_{i}^{\dagger} \mapsto-\delta_{\left|e_{a}\right|, 0} \delta_{\left|f_{i}^{\dagger}\right|, 0} \sum_{k=0}^{M-1} \operatorname{Res}\left[\frac{\left(\begin{array}{c}
{\left[\partial_{X} \mathrm{~d}_{0}\right]^{X \rightarrow X^{\prime \prime}} \cdot \eta_{r, 0}^{-a} \cdot \partial^{X, \eta^{-a} X^{\prime \prime} \mathrm{d}_{1}+}} \\
+\eta^{a}\left[\partial^{X, X^{\prime \prime}} \mathrm{d}_{0}\right]^{X \rightarrow \eta^{a} X} \cdot\left[\partial_{X} \mathrm{~d}_{1}\right]^{X \rightarrow X^{\prime \prime}} \cdot \eta_{r, 1}^{-a}
\end{array}\right)_{i, k}^{Z \rightarrow Z^{\prime}} \mathrm{d} X^{\prime \prime}}{d \cdot X^{\prime \prime} d^{-1}}\right] f_{k}^{\dagger} \\
& -\delta_{\left|e_{a}\right|, 0} \delta_{\left|f_{i}^{\dagger}\right|, 1} \sum_{k=M}^{2 M-1} \operatorname{Res}\left[\frac{\left(\begin{array}{c}
{\left[\partial_{X} \mathrm{~d}_{1}\right]^{X \rightarrow X^{\prime \prime}} \cdot \eta_{r, 1}^{-a} \cdot \partial^{X, \eta^{-a} X^{\prime \prime} \mathrm{d}_{0}+}} \\
+\eta^{a}\left[\partial^{X, X^{\prime \prime}} \mathrm{d}_{1}\right]^{X \rightarrow \eta^{X} X} \cdot\left[\partial_{X} \mathrm{~d}_{0}\right]^{X \rightarrow X^{\prime \prime}} \cdot \eta_{r, 0}^{-a}
\end{array}\right)_{i-M, k-M}^{Z \rightarrow Z^{\prime}} \mathrm{d} X^{\prime \prime}}{d \cdot X^{\prime \prime} d^{-1}}\right] f_{k}^{\dagger}
\end{aligned}
$$

for the following choice of variables

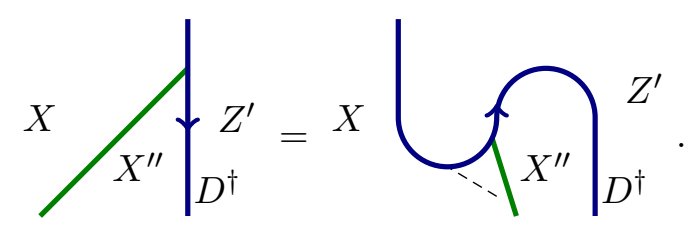


Here $\eta=e^{2 \pi i / d}$, and $\eta_{r, 0}$ and $\eta_{r, 1}$ are the diagonal matrices

$$
\begin{aligned}
& \eta_{r, 0}=\operatorname{diag}\left(\eta^{r_{0}}, \ldots, \eta^{r_{M-1}}\right) \\
& \eta_{r, 1}=\operatorname{diag}\left(\eta^{r_{M}}, \ldots, \eta^{r_{2 M-1}}\right) .
\end{aligned}
$$

Moreover, $\partial^{X, X^{\prime \prime}}$ is the divided difference operator which is defined as $\partial^{X, X^{\prime \prime}} g(X, \ldots)=$ $\frac{g(X, \ldots)-g\left(X^{\prime \prime}, \ldots\right)}{X-X^{\prime \prime}}$ on any polynomial $g$, and the residue Res $\left[\frac{g \cdot d X^{\prime \prime}}{X^{\prime \prime d-1}}\right]$ picks out the prefactor of $X^{\prime \prime d-2}$ in the polynomial $g \in \mathbb{C}\left[Z^{\prime}, X, X^{\prime \prime}\right]$.

We now simplify expression (C.6) by calculating the $X^{\prime \prime}{ }^{d-2}$-term in the numerator. We first derive a few identities which follow from the very definition of a graded matrix factorization.

From the basic property of matrix factorizations $\mathrm{d}_{0} \mathrm{~d}_{1}=\left(Z^{d^{\prime}}-X^{d}\right) \mathbb{1}$ one can deduce

$$
\begin{aligned}
\partial^{X, X^{\prime \prime}}\left(-d \cdot X^{d-1}\right)= & {\left[\left.\partial_{X} \mathrm{~d}_{0}\right|^{X \rightarrow X^{\prime \prime}} \cdot \partial^{X, X^{\prime \prime}} \mathrm{d}_{1}+\partial^{X, X^{\prime \prime}} \mathrm{d}_{0} \cdot\left[\left.\partial_{X} \mathrm{~d}_{1}\right|^{X \rightarrow X^{\prime \prime}}\right.\right.} \\
& +\left\{\left(\partial^{X, X^{\prime \prime}} \partial_{X} \mathrm{~d}_{0}\right) \cdot \mathrm{d}_{1}+\mathrm{d}_{0} \cdot\left(\partial^{X, X^{\prime \prime}} \partial_{X} \mathrm{~d}_{1}\right)\right\} .
\end{aligned}
$$

Now, we will simplify the derivation by assuming that the matrices $d_{0}$ and $d_{1}$ do not contain terms $X^{n}$ for $n \geq d$. This is certainly true for all the matrix factorizations relevant in this paper, namely the ones associated to RG and projection defects, boundary conditions etc. Under this assumption, the curly bracket part of the last equation does not contain a term $\sim X^{\prime \prime d-2}$, and hence

$$
\left\{\left[\partial_{X} \mathrm{~d}_{0}\right]^{X \rightarrow X^{\prime \prime}} \cdot \partial^{X, X^{\prime \prime}} \mathrm{d}_{1}+\partial^{X, X^{\prime \prime}} \mathrm{d}_{0} \cdot\left[\left.\partial_{X} \mathrm{~d}_{1}\right|^{X \rightarrow X^{\prime \prime}}\right\}_{i k}=-d X^{\prime \prime d-2} \delta_{i k}+\ldots\right.
$$

where ... contains only powers $\left(X^{\prime \prime}\right)^{n}$ with $n<d-2$. In order to make contact with equation (C.6) we replace $\partial^{X, X^{\prime \prime}} \mathrm{d}_{0}$ in (C.7) by $\left.\partial^{X, X^{\prime \prime}} \mathrm{d}_{0}\right|^{X \rightarrow \eta^{a} X}$ which does not alter the leading $X^{\prime \prime}$-term:

$$
\left\{\left[\partial_{X} \mathrm{~d}_{0}\right]^{X \rightarrow X^{\prime \prime}} \cdot \partial^{X, X^{\prime \prime}} \mathrm{d}_{1}+\left.\partial^{X, X^{\prime \prime}} \mathrm{d}_{0}\right|^{X \rightarrow \eta^{a} X} \cdot\left[\left.\partial_{X} \mathrm{~d}_{1}\right|^{X \rightarrow X^{\prime \prime}}\right\}_{i k}=-d X^{\prime \prime d-2} \delta_{i k}+\ldots\right.
$$

Also, since $d_{1}$ is of grade zero

$$
\eta_{r, 0}^{-a} \cdot\left[\left.\mathrm{d}_{1}\right|^{X \rightarrow \eta^{-a} X}=\mathrm{d}_{1} \cdot \eta_{r, 1}^{-a}\right.
$$

which together with the definition of the divided difference operator yields

$$
\left.\eta_{r, 0}^{-a} \cdot \partial^{X, \eta^{-a} X^{\prime \prime}} \mathrm{d}_{1}\right|_{X^{\prime \prime} \text {-term }} ^{\text {leading }}=\left.\partial^{X, X^{\prime \prime}} \mathrm{d}_{1}\right|_{X_{X^{\prime \prime} \text {-term }}^{\text {leading }}} \cdot \eta^{a} \cdot \eta_{r, 1}^{-a} .
$$

Here, $\left.\partial^{X, \eta^{-a} X^{\prime \prime}} \mathrm{d}_{1}\right|_{\substack{\text { leading } \\ X^{\prime \prime} \text {-term }}}$ is the matrix $\mathrm{d}_{1}$ with all entries $X^{p+1}$ replaced by $\left(\eta^{-a} \cdot X^{\prime \prime}\right)^{p}$ 
and similarly for $\left.\partial^{X, X^{\prime \prime}} \mathrm{d}_{1}\right|_{\substack{\text { leading } \\ X^{\prime \prime} \text {-term }}}$. Finally, we evaluate the first summand of (C.6):

$$
\begin{aligned}
& -\delta_{\left|e_{a}\right|, 0} \delta_{\left|f_{i}^{\dagger}\right|, 0} \sum_{k=0}^{M-1} \operatorname{Res}\left[\frac{\left(\begin{array}{c}
{\left[\partial_{X} \mathrm{~d}_{0}\right]^{X \rightarrow X^{\prime \prime}} \cdot \eta_{r, 0}^{-a} \cdot \partial^{X, \eta^{-a} X^{\prime \prime} \mathrm{d}_{1}+}} \\
+\eta^{a}\left[\partial^{X, X^{\prime \prime}} \mathrm{d}_{0}\right]^{X \rightarrow \eta^{a} X} \cdot\left[\partial_{X} \mathrm{~d}_{1}\right]^{X \rightarrow X^{\prime \prime}} \cdot \eta_{r, 1}^{-a}
\end{array}\right)_{i, k} \mathrm{~d} X}{d \cdot X^{\prime \prime}{ }^{d-1}}\right]^{Z \rightarrow Z^{\prime}} f_{k}^{\dagger} \\
& \stackrel{(\mathrm{C} .9)}{=}-\delta_{\left|e_{a}\right|, 0} \delta_{\left|f_{i}^{\dagger}\right|, 0} \sum_{k=0}^{M-1} \operatorname{Res}\left[\frac{\left(\begin{array}{c}
{\left[\partial_{X} \mathrm{~d}_{0}\right]^{X \rightarrow X^{\prime \prime}} \cdot \partial^{X, X^{\prime \prime}} \mathrm{d}_{1} \cdot \eta^{a} \cdot \eta_{r, 1}^{-a}+\ldots} \\
+\eta^{a}\left[\partial^{X, X^{\prime \prime}} \mathrm{d}_{0}\right]^{X \rightarrow \eta^{a} X} \cdot\left[\partial_{X} \mathrm{~d}_{1}\right]^{X \rightarrow X^{\prime \prime}} \cdot \eta_{r, 1}^{-a}
\end{array}\right)_{i, k} \mathrm{~d} X}{d \cdot X^{\prime \prime}{ }^{d-1}}\right]^{Z \rightarrow Z^{\prime}} f_{k}^{\dagger} \\
& =-\delta_{\left|e_{a}\right|, 0} \delta_{\left|f_{i}^{\dagger}\right|, 0} \sum_{k=0}^{M-1} \operatorname{Res}\left[\frac{\left(\begin{array}{c}
{\left[\partial_{X} \mathrm{~d}_{0}\right]^{X \rightarrow X^{\prime \prime}} \cdot \partial^{X, X^{\prime \prime}} \mathrm{d}_{1}+} \\
+\left[\partial^{X, X^{\prime \prime}} \mathrm{d}_{0}\right]^{X \rightarrow \eta^{a} X} \cdot\left[\partial_{X} \mathrm{~d}_{1}\right]^{X \rightarrow X^{\prime \prime}}
\end{array}\right)_{i, k} \cdot \eta^{-a\left(r_{k+M}-1\right)} \mathrm{d} X}{d \cdot X^{\prime \prime}{ }^{d-1}}\right]^{Z \rightarrow Z^{\prime}} f_{k}^{\dagger} \\
& \stackrel{(\mathrm{C} .8)}{=}-\delta_{\left|e_{a}\right|, 0} \delta_{\left|f_{i}^{\dagger}\right|, 0} \sum_{k=0}^{M-1} \operatorname{Res}\left[\frac{\left(-d X^{\prime \prime}{ }^{d-2} \delta_{i k}+\ldots\right) \mathrm{d} X}{d \cdot X^{\prime \prime} d-1}\right]^{Z \rightarrow Z^{\prime}} \cdot \eta^{-a\left(r_{k+M}-1\right)} f_{k}^{\dagger} \\
& =\delta_{\left|e_{a}\right|, 0} \delta_{\left|f_{i}^{\dagger}\right|, 0} \eta^{a\left(-r_{i+M}+1\right)} f_{i}^{\dagger} .
\end{aligned}
$$

Here, with '...' we indicate that we omitted terms which do not contribute to the residue.

The second summand in equation (C.6) can be determined in a similar way and also takes a similar form. We find that the left charges of $D^{\dagger}$ are the negative right charges of $D$ shifted by +1 :

$$
\begin{aligned}
& A \otimes D^{\dagger} \rightarrow D^{\dagger} \\
& e_{a} \otimes f_{i}^{\dagger} \mapsto \delta_{\left|e_{a}\right|, 0} \eta^{a\left(-r_{i+M}+1\right)} f_{i}^{\dagger} .
\end{aligned}
$$

\section{C.8 (Co)evaluation maps}

Finally, we provide the explicit (co)evaluation maps used in calculations in the main text. They follow from the generalized orbifold construction, (cf. appendix B) and the expressions of [12]. Throughout, [...] denotes the representative in $\{0, \ldots, d-1\}$ modulo d. Furthermore, $\partial^{Z, X} Z^{i}=\frac{Z^{i}-X^{i}}{Z-X}, \sigma=\left(\begin{array}{cc}\mathbb{1} & 0 \\ 0 & -\mathbb{1}\end{array}\right), \eta=e^{\frac{2 \pi i}{d}}, \epsilon=e^{\frac{2 \pi i}{d^{\prime}}}, \eta_{r}=\operatorname{diag}\left(\eta^{r_{0}}, \eta^{r_{1}}, \eta^{r_{2}}, \ldots\right)$, $\epsilon_{l}=\operatorname{diag}\left(\epsilon^{l_{0}}, \epsilon^{l_{1}}, \epsilon^{l_{2}}, \ldots\right)$ and $\epsilon_{1+l}=\operatorname{diag}\left(\epsilon^{1+l_{0}}, \epsilon^{1+l_{1}}, \epsilon^{1+l_{2}}, \ldots\right)$.

\section{Orbifold evaluation map (left)}

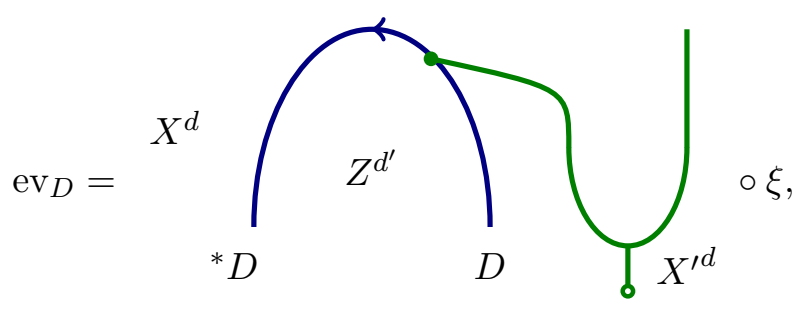


where $\xi:{ }^{*} D \otimes_{A} D \rightarrow{ }^{*} D \otimes D$ is the inclusion.

$$
\begin{aligned}
& e v_{D}:{ }^{*} D \otimes_{A} D \rightarrow A \\
& { }^{*} f_{k} \otimes Z^{n} f_{i} \mapsto \frac{1}{d} \sum_{h \in \mathbb{Z}_{d}} \operatorname{Res}\left[\frac{Z^{n}\left(\sigma \cdot \partial_{Z} \mathrm{~d}_{D} \cdot \eta_{r}^{h}\right)_{(k+M), i}}{d^{\prime} \cdot Z^{d^{\prime}-1}}\right] e_{[h]} \\
& \quad+\frac{1}{d} \sum_{j} \sum_{h \in \mathbb{Z}_{d}} \operatorname{Res}\left[\frac{Z^{n}\left(\sigma \cdot \partial_{Z} \mathrm{~d}_{D} \cdot \eta_{r}^{h} \cdot\left[\partial^{X, X^{\prime}} \mathrm{d}_{D}\right]^{X \rightarrow \eta^{h} X} \cdot \sigma\right)_{(k+M), i}}{d^{\prime} \cdot Z^{d^{\prime}-1}}\right] e_{d+[h]}
\end{aligned}
$$

\section{Orbifold evaluation map (right)}

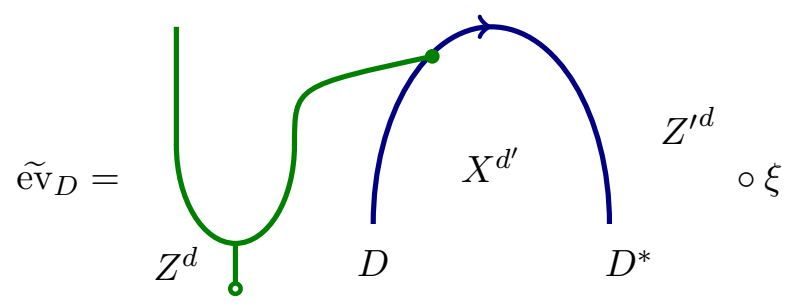

where $\xi: D \otimes_{A} D^{*} \rightarrow D \otimes D^{*}$ is the inclusion.

$$
\begin{aligned}
& \widetilde{\mathrm{ev}}_{D}: D \otimes{ }_{A} D^{*} \rightarrow A \\
& f_{i} \otimes X^{n} f_{k}^{*} \mapsto-\frac{1}{d^{\prime}} \sum_{h \in \mathbb{Z}_{d^{\prime}}} e_{[-h]} \operatorname{Res}\left[\frac{\left(\partial_{X} \mathrm{~d}_{D} \cdot \epsilon_{l}^{h}\right)_{(k+M), i}^{Z \rightarrow Z^{\prime}} X^{n}}{d \cdot X^{d-1}}\right] \\
&-\frac{1}{d^{\prime}} \sum_{h \in \mathbb{Z}_{d^{\prime}}} e_{d^{\prime}+[-h]} \operatorname{Res}\left[\frac{\left(\partial_{X} \mathrm{~d}_{D}^{Z \rightarrow Z^{\prime}} \cdot \epsilon_{1+l}^{h} \cdot \partial^{Z, \epsilon^{h} Z^{\prime}} \mathrm{d}_{D}\right)_{(k+M), i} X^{n}}{d \cdot X^{d-1}}\right]
\end{aligned}
$$

\section{Orbifold coevaluation map (left)}

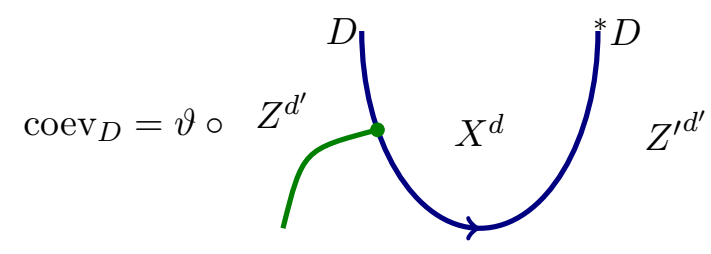

where $\vartheta: D \otimes{ }^{*} D \rightarrow D \otimes_{A}{ }^{*} D$ is the projection.

$$
\begin{aligned}
\operatorname{coev}_{D}: A & \rightarrow D \otimes_{A}{ }^{*} D \\
e_{a} \mapsto & \delta_{\left|e_{a}\right|, 0} \sum_{i j}(-1)^{\left|e_{j}\right|}\left(\left\{\partial^{Z, Z^{\prime}} \mathrm{d}_{D}\right\}^{Z \mapsto \epsilon^{a} Z}\right)_{i j} \epsilon^{a l_{i}} f_{i} \otimes f_{(j+M)}^{*} \\
& +\delta_{\left|e_{a}\right|, 1} \sum_{i}(-1)^{\left|e_{i}\right|} \epsilon^{a l_{i}} f_{i} \otimes f_{(i+M)}^{*}
\end{aligned}
$$


Orbifold coevaluation map (right)

$$
\widetilde{\operatorname{cov}}_{D}=\vartheta \circ X_{X^{d^{\prime}}}^{D^{*}} \underbrace{D}_{X^{\prime d^{\prime}}}
$$

where $\vartheta: D \otimes D^{*} \rightarrow D \otimes_{A} D^{*}$ is the projection.

$$
\begin{aligned}
\widetilde{\operatorname{coev}}_{D}: A \rightarrow & D^{*} \otimes D \\
e_{a} \mapsto & \delta_{\left|e_{a}\right|, 0} \sum_{i j}\left(\partial^{X, \eta^{-a} X^{\prime}} \mathrm{d}_{D}\right)_{j i} f_{(i+M)}^{*} \otimes f_{j} \eta^{-a r_{j}} \\
& +\delta_{\left|e_{a}\right|, 1} \eta^{a} \sum_{i}(-1)^{\left|e_{i}\right|} f_{(i+M)}^{*} \otimes f_{i} \eta^{-a r_{i}}
\end{aligned}
$$

Orbifold evaluation map (right) for boundaries

$$
\widetilde{\mathrm{ev}}_{B}=\overbrace{B} X_{B^{*}}
$$

$\widetilde{\mathrm{ev}}_{B}: B \otimes_{A} B^{*} \rightarrow \mathbb{C}$

$$
f_{i} \otimes X^{p} f_{k}^{*} \mapsto-\operatorname{Res}\left[\frac{X^{p}\left(\partial_{X} \mathrm{~d}_{B}\right)_{(k+M), i} \mathrm{~d} X}{d \cdot X^{d-1}}\right]
$$

Orbifold coevaluation map (left) for boundaries

$$
\begin{aligned}
\operatorname{coev}_{B}= & \left.\left.\right|_{\operatorname{cov}_{B}:} ^{B}\right|^{*} \rightarrow B \otimes_{A}{ }^{*} B \\
1 & \mapsto \sum_{i} f_{i} \otimes{ }^{*} f_{i}
\end{aligned}
$$

\section{Explicit calculations for RG defects in LG orbifolds}

In this appendix we explicitly check that the RG defects $R$ between LG orbifolds presented in section 4 satisfy the RG property that $R \otimes R^{\dagger} \cong I$ (appendix D.1) and determine the corresponding projection defects $P=R^{\dagger} \otimes R$ (appendix D.2). We show how IR boundary conditions and symmetries are realized in the UV (appendices D.3 and D.4) and we perform the calculation $R_{\infty} \otimes R_{\infty}^{\dagger} \cong I_{\mathrm{IR}}$ (appendix D.5). For the purpose of this appendix we again adopt the generalized orbifold notation of appendix $\mathrm{C}$. 


\section{D.1 $R \otimes_{A} R^{*} \cong A$}

Here, we show that $R \otimes_{A} R^{*} \cong A$. (In this appendix we adopt the following notation from appendix $\mathrm{C}: \otimes_{A}$ denotes fusion in the generalized orbifold theory defined by $A$, while $\otimes$ denotes the fusion in the unorbifolded theory. Moreover, ${ }^{*}$ denotes adjunction in the orbifold theory, while ${ }^{\dagger}$ refers to adjunction in the unorbifolded theory.) Fusion of B-type defects has been discussed in [13], for the orbifold version see [1].

As explained in those papers, matrix factorizations of $W$ over a polynomal ring $R$ are related to finitely generated modules over $\hat{R}:=R /(W)$ as free resolutions of such modules always turn two-periodic after finitely many steps [41]. The two-periodic part then gives a matrix factorization of $W$.

In order to calculate $R \otimes_{A} R^{*}$, we fix the coordinates on all three parts of the worldsheet to be $Z, X$ and $Y$ :

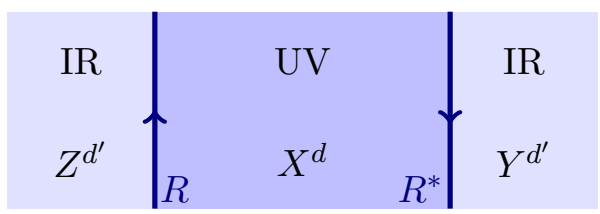

The matrix factorization describing $R$ is given by

$$
\begin{aligned}
& \mathrm{d}_{R 1}=\left(\begin{array}{ccccc}
Z & 0 & \cdots & 0 & -X^{n_{0}} \\
-X^{n_{1}} & Z & & & \\
0 & -X^{n_{2}} & Z & & \\
\vdots & & \ddots & \ddots & \\
0 & & & -X^{n_{d^{\prime}-1}} & Z
\end{array}\right) \\
& R: R_{1} \rightleftarrows \mathrm{d}_{R 0} \rightleftarrows R_{0} \text {, }
\end{aligned}
$$

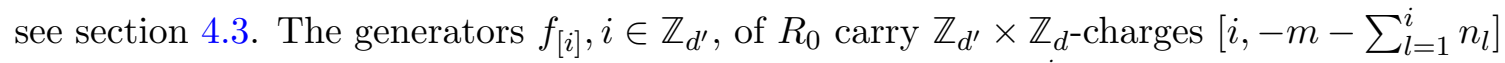
while the generators $e_{d^{\prime}+[i]}$ of $R_{1}$ have charges $\left[i+1,-m-\sum_{l=1}^{i} n_{l}\right]$.

According to section 4.2 , the right adjoint $R^{*}$ is given by the matrix factorization

$$
\mathrm{d}_{R^{*} 1}=\left(\begin{array}{ccccc}
Y & -X^{n_{1}} & 0 & \cdots & 0 \\
0 & Y & -X^{n_{2}} & & \\
\vdots & & Y & \ddots & \\
0 & & & \ddots & -X^{n_{d^{\prime}-1}} \\
-X^{n_{0}} & & & & Y
\end{array}\right)
$$

The generators $f_{[k]}^{*}, k \in \mathbb{Z}_{d^{\prime}}$, of $R_{0}^{*}$ carry $\mathbb{Z}_{d} \times \mathbb{Z}_{d^{\prime}}$-charges $\left[+m+\sum_{l=1}^{k} n_{l}+1,-k-1\right]$, and the generators $f_{d^{\prime}+[k]}^{*}$ of $R_{1}^{*}$ carry charges $\left[+m+\sum_{l=1}^{k} n_{l}+1,-k-1\right]$. 
Following the tensor product formula of section 4.1, the matrix factorization describing

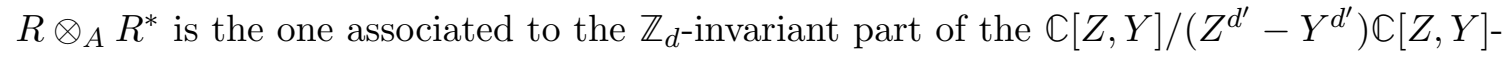
module

$$
M:=\operatorname{coker}\left(\begin{array}{cc}
\mathrm{id}_{R 0} \otimes \mathrm{d}_{R^{*} 1} & \mathrm{~d}_{R 1} \otimes \mathrm{id}_{R^{*} 0} \\
\mathrm{~d}_{R 0} \otimes \mathrm{id}_{R^{*} 1} & -\mathrm{id}_{R 1} \otimes \mathrm{d}_{R^{*} 0}
\end{array}\right) .
$$

The two-periodic resolution of $M$ is isomorphic to the two-periodic part of the resolution of

$$
M^{\prime}:=\operatorname{coker}\left(\mathrm{d}_{R 1} \otimes \operatorname{id}_{R^{*} 0}, \operatorname{id}_{R 0} \otimes \mathrm{d}_{R^{*} 1}\right) .
$$

The module $M^{\prime}$ is generated by $f_{[i],[k]}^{l}:=f_{[i]} \otimes X^{l} f_{[k]}^{*}$. They satisfy the relations

$$
Z f_{[i]}=X^{n_{i+1}} f_{[i+1]} \quad \text { and } \quad Y f_{[k]}^{*}=X^{n_{[k]}} f_{[k-1]}^{*},
$$

which allow to reduce the generators to $f_{[i],[k]}^{l}$ with $0 \leq l<\min \left(n_{i}, n_{k+1}\right)$. These carry $\mathbb{Z}_{d^{\prime}} \times \mathbb{Z}_{d} \times \mathbb{Z}_{d^{\prime}}$ charges

$$
\left[i,-m-\sum_{j=1}^{i} n_{j}+l+m+\sum_{j=1}^{k} n_{j}+1,-k-1\right] .
$$

The $\mathbb{Z}_{d}$-invariant part $\left(M^{\prime}\right)^{\mathbb{Z}_{d}}$ is generated by the $\mathbb{Z}_{d}$-invariant generators of $M^{\prime}$, which are given by $\hat{f}_{[i]}:=f_{[i],[i-1]}^{n_{i}-1}$. They carry $\mathbb{Z}_{d^{\prime}} \times \mathbb{Z}_{d^{\prime}}$-charges $[i,-i]$ and satisfy the relations

$$
Z \hat{f}_{[i]}=Y \hat{f}_{[i+1]} .
$$

Hence, $\left(M^{\prime}\right)^{\mathbb{Z}_{d}}$ is isomorphic to the module coker $\left(\mathrm{d}_{A 1}\right)$, which implies that the matrix factorization $R \otimes_{A} R^{*}$ is isomorphic to the identity defect $A$ in $\mathcal{M}_{d^{\prime}} / \mathbb{Z}_{d^{\prime}}$. Taking the left adjoint of this equation immediately yields $R \otimes_{A}{ }^{*} R \cong A$ as well.

\section{D.2 The projection defect $P$}

Having shown $R \otimes_{A} R^{*} \cong A$ in the previous appendix, we are now in a position to determine the projection defect $P=R^{*} \otimes_{A} R$. The projection $P^{\prime}={ }^{*} R \otimes_{A} R$ based on the left adjoint ${ }^{*} R$ can then easily be obtained by left adjunction $P^{\prime}={ }^{*} P$.

The calculation of $P$ follows the same route as the calculation of $R \otimes_{A} R^{*}$ in appendix D.1 above. First, we fix the chiral fields on all three parts of the worldsheet to be $Y, Z$ and $X$ :

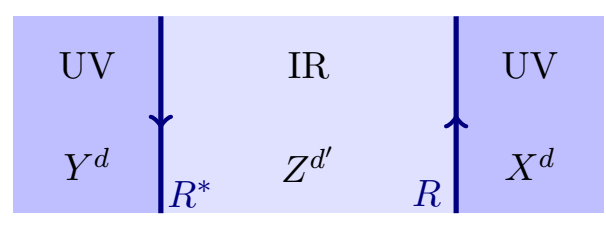

The matrix factorizations $R$ and $R^{*}$ are described in appendix D.1. As in the derivation of $R \otimes_{A} R^{*} \cong I$, the matrix factorization $R^{*} \otimes R$ is given by the two-periodic part of the free resolution of the $\mathbb{Z}_{d^{\prime}}$-invariant part of the $\mathbb{C}[X, Y] /\left(Y^{d}-X^{d}\right) \mathbb{C}[X, Y]$-module

$$
M^{\prime}:=\operatorname{coker}\left(d_{R^{*} 1} \otimes \operatorname{id}_{R_{0}}, \operatorname{id}_{R^{*} 0} \otimes d_{R 1}\right) .
$$


The latter is generated by

$$
f_{[k],[i]}^{l}:=f_{[k]}^{*} Z^{l} \otimes f_{[i]}
$$

subject to the relations

$$
Y^{n_{k}} f_{[k-1]}^{*}=Z f_{[k]}^{*} \quad \text { and } \quad Z f_{[i]}=X^{n_{i+1}} f_{[i+1]} .
$$

These relations allow to reduce the generators to the ones with $l=0$. The remaining generators $f_{[k],[i]}^{0}$ carry $\mathbb{Z}_{d} \times \mathbb{Z}_{d^{\prime}} \times \mathbb{Z}_{d^{\text {-charges }}}$

$$
\left[m+\sum_{j=1}^{[k]} n_{j}+1,-k-1+i,-m-\sum_{j=1}^{[i]} n_{j}\right] .
$$

The $\mathbb{Z}_{d^{\prime}}$-invariant part of $M^{\prime}$ is generated by the $\mathbb{Z}_{d^{\prime}}$-invariant generators, i.e. those $f_{[k],[i]}^{0}$, for which $[-k-1+i]=0$. These are the $\hat{f}_{[i]}:=f_{[i-1],[i]}^{0}$, which are subject to the relations

$$
Y^{n_{i}} \hat{f}_{[i]}=X^{n_{i+1}} \hat{f}_{[i+1]}
$$

They carry $\mathbb{Z}_{d} \times \mathbb{Z}_{d}$-charges

$$
\left[m+\sum_{j=1}^{[i-1]} n_{j}+1,-m-\sum_{j=1}^{[i]} n_{j}\right] .
$$

Comparing with the matrix factorization $P$ given in equation (4.4), one finds that $\left(\widetilde{M}^{\prime}\right)^{\mathbb{Z}_{d^{\prime}}} \cong$ $\operatorname{coker}\left(p_{1}\right)$ (where $Z$ has to be replaced by $Y$ in $p_{1}$ ). Hence, $R^{*} \otimes R$ is isomorphic to the matrix factorization $P$ given in section 4.3.

\section{D.3 Boundary conditions satisfying $B \otimes_{A} P \cong B$}

We now determine the boundary conditions, which are invariant under fusion with $P$. Elementary left boundary conditions in $\mathcal{M}_{d} / \mathbb{Z}_{d}$ are given by the $\mathbb{Z}_{d}$-equivariant matrix factorizations

$$
B_{\mathrm{UV}}: \mathbb{C}[Z]([N+k]) \underset{-Z^{d-k}}{\longleftarrow} \mathbb{C}[Z]([N]) .
$$

of $-Z^{d}$, where $k, N \in \mathbb{Z}_{d}, k \neq 0$, cf. section 4.4. The aim is to identify those boundary conditions, for which $B_{\mathrm{UV}} \otimes_{A} P \cong B_{\mathrm{UV}}$. To do so, we just calculate the fusion as is done in the previous appendices. We denote the generators of $B_{0}$ and $B_{1}$ by $b_{0}$ and $b_{1}$, respectively.

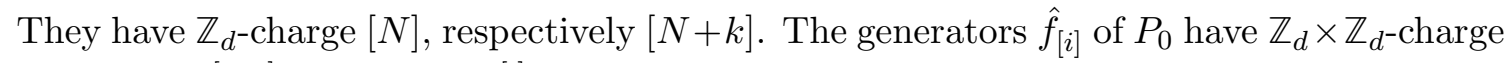
$\left[m+1+\sum_{l=1}^{[i-1]} n_{[l]},-m-\sum_{l=1}^{[i]} n_{[l]}\right]$, cf. appendix D.2.

To determine the fusion $B_{\mathrm{UV}} \otimes P$, we again employ the method described in appendix D.1. For this, we determine generators and relations of the $\mathbb{Z}_{d}$-invariant part of the $\mathbb{C}[X] / X^{d} \mathbb{C}[X]$-module $M^{\prime}:=\operatorname{coker}\left(d_{B 1} \otimes \operatorname{id}_{P 0}, \operatorname{id}_{B 0} \otimes d_{P 1}\right):$

$$
\begin{array}{r}
b_{0} Z^{k}=0 \\
Z^{n_{i}} f_{[i]}=X^{n_{[i+1]}} f_{[i+1]}
\end{array}
$$


For $B_{\mathrm{UV}} \otimes_{A} P \cong B_{\mathrm{UV}}$ to hold, out of all the generators $b_{0} Z^{q} \otimes f_{[i]}$ of the fusion product exactly one generator may survive in $\left(M^{\prime}\right)^{\mathbb{Z}_{d}}$. It must

- be invariant under the left $\mathbb{Z}_{d}$-action, i.e.

$$
\left[N+q+m+1+\sum_{l=1}^{[i-1]} n_{l}\right]=0
$$

- carry right $\mathbb{Z}_{d^{-}}$charge $[N]$, i.e.

$$
\left[-m-\sum_{l=1}^{[i]} n_{l}\right]=[N]
$$

- has to be a generator with respect to $\mathbb{C}[X]$ and in particular cannot be eliminated by (D.1), i.e.

$$
q<n_{i} \quad \text { and } \quad q<k
$$

and

- it has to satisfy $b_{0} Z^{q} \otimes f_{[i]} X^{k}=0$.

The first two conditions fix $N=-m-\sum_{j=1}^{[i]}$ and imply $q=n_{i}-1$ which is consistent with $q<n_{i}$. The last condition becomes

$$
k \in\left\{n_{i}, n_{i}+n_{i-1}, \ldots, n_{i}+\ldots+n_{i-d^{\prime}-2}\right\} .
$$

These conditions are equivalent to $B_{\mathrm{UV}} \otimes_{A} P \cong B_{\mathrm{UV}}$ and imply that $B_{\mathrm{UV}}$ must be of the form

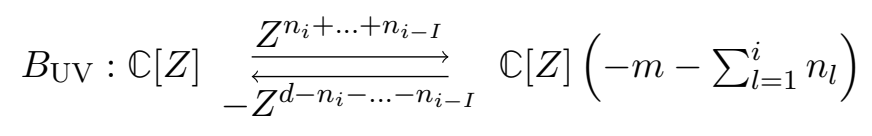

for arbitrary $i \in \mathbb{Z}_{d^{\prime}}$ and $I \in\left\{0, \ldots, d^{\prime}-2\right\}$.

\section{D.4 IR symmetry defects in the UV}

Following section 4.4 , the IR $\mathbb{Z}_{d^{\prime}}$-symmetry is realized in the UV by means of the defects

$$
R^{*} \otimes_{A a_{a} I_{d^{\prime}}} \otimes_{A} R=:{ }_{a} P
$$

As ${ }_{a} I_{d^{\prime}} \otimes_{A} R$ is described by the same matrix factorization as $R$ but with all left charges shifted by $+a$, we can employ the same set-up as in appendix D.2 and only shift charges by $+a$ where necessary. The corresponding module $M^{\prime}$ is generated by

$$
f_{[k],[i]}^{l}:=f_{[k]}^{*} Z^{l} \otimes f_{[i]}
$$


with $\mathbb{Z}_{d} \times \mathbb{Z}_{d^{\prime}} \times \mathbb{Z}_{d^{\text {-charges }}}$

$$
\left[m+\sum_{j=1}^{[k]} n_{j}+1,-k-1+l+i+a,-m-\sum_{j=1}^{[i]} n_{j}\right]
$$

subject to the relations

$$
Y^{n_{k}} f_{[k-1]}^{*}=Z f_{[k]}^{*} \quad \text { and } \quad Z f_{[i]}=X^{n_{i+1}} f_{[i+1]}
$$

While the relations can be used to reduce generators to those with $l=0, \mathbb{Z}_{d^{\prime}}$-invariance gives the condition $[i+a-k-1]=0$. The remaining generators $\hat{f}_{[i]}:=f_{[i-1],[i-a]}^{0}$ of $\left(M^{\prime}\right)^{\mathbb{Z}_{d^{\prime}}}$ obey

$$
Y^{n_{i}} \hat{f}[i]=X^{n_{i-a+1}} f_{[i+1]}
$$

and carry $\mathbb{Z}_{d} \times \mathbb{Z}_{d^{-}}$-charges

$$
\left[m+\sum_{j=1}^{[i-1]} n_{j}+1,-m-\sum_{j=1}^{[i-a]} n_{j}\right]
$$

One now easily reads off that $\left(M^{\prime}\right)^{\mathbb{Z}_{d^{\prime}}}$ is isomorphic to the cokernel of the matrix $p_{1}$ of the matrix factorization ${ }_{a} P$ given in section 4.3. Thus, the lifted symmetry defects are isomorphic to these matrix factorizations.

\section{D.5 $R_{\infty} \otimes_{\mathrm{U}(1)} R_{\infty}^{*} \cong I_{\mathrm{IR}}$}

In this appendix we show that one can insert loops of the U(1)-equivariant LandauGinzburg theory with a single chiral superfield and zero superpotential into the LandauGinzburg orbifold models $\mathcal{M}_{d^{\prime}} / \mathbb{Z}_{d^{\prime}}, d^{\prime} \geq 3$ without affecting correlators. The respective RG defects are described by the matrix factorizations $R_{\infty}$ of $Z^{d^{\prime}}$ presented in section 4.5 :

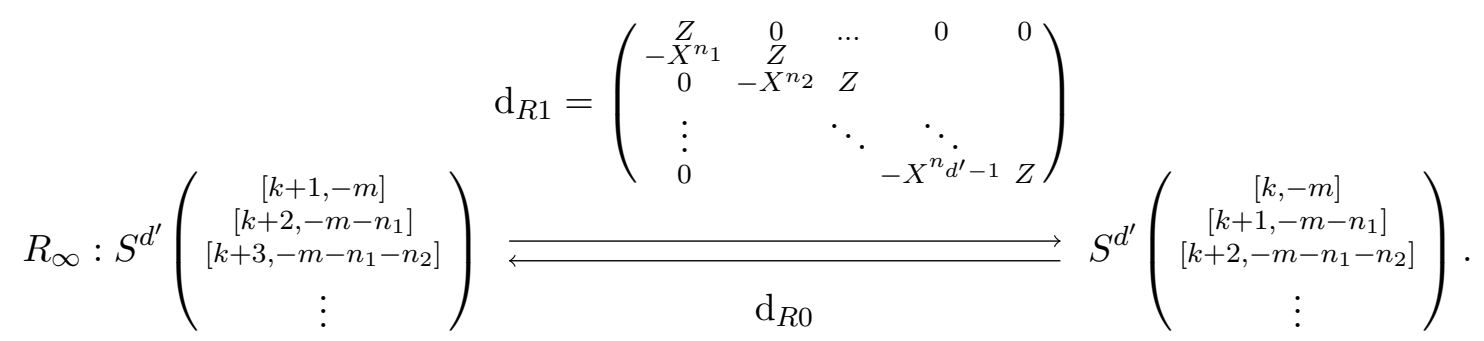

Here $m \in \mathbb{Z}, k \in \mathbb{Z}_{d^{\prime}}$ and $n_{1}, \ldots, n_{d^{\prime}-1} \in \mathbb{N}$. Moreover, $S=\mathbb{C}[Z, X]$ and

$$
\mathrm{d}_{R 0}=\left(\begin{array}{ccccc}
Z^{d^{\prime}-1} & 0 & \cdots & \cdots & 0 \\
Z^{d^{\prime}-2} X^{n_{1}} & Z^{d^{\prime}-1} & 0 & \cdots & 0 \\
Z^{d^{\prime}-3} X^{n_{1}+n_{2}} & Z^{d^{\prime}-2} X^{n_{2}} & Z^{d^{\prime}-1} & \ddots & \vdots \\
\vdots & \vdots & \ddots & \ddots & 0 \\
X^{n_{1}+\ldots+n_{d^{\prime}-1}} & Z X^{n_{2}+\ldots+n_{d^{\prime}-1}} & \ldots & Z^{d^{\prime}-2} X^{n_{d^{\prime}-1}} & Z^{d^{\prime}-1}
\end{array}\right) .
$$


The adjoint

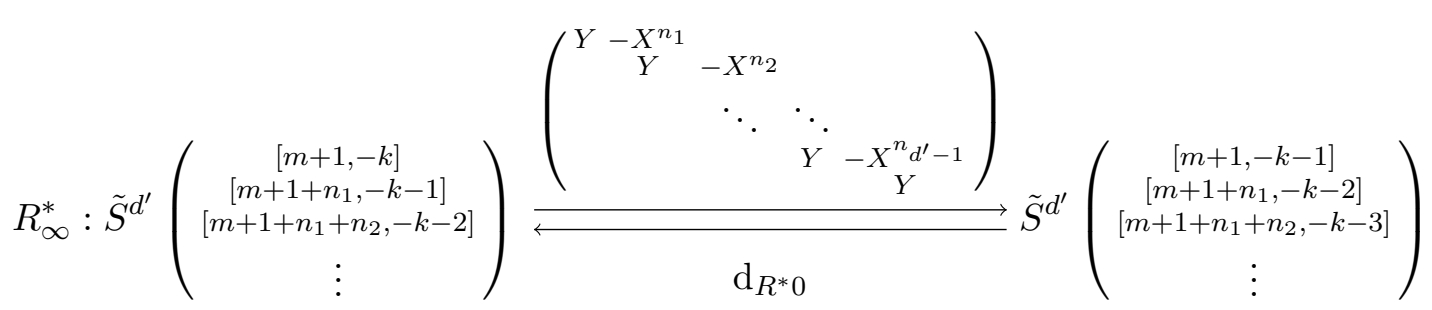

can be obtained by taking the limit $d \rightarrow \infty$ of $R^{*}$. It is a matrix factorization of $-Y^{d^{\prime}}$. $\tilde{S}=\mathbb{C}[X, Y]$ and $\mathrm{d}_{R^{*} 0}$ is given by $-\mathrm{d}_{R 0}^{T}$ with $Z$ replaced by $Y$.

According to section 4.1, the fusion product $R_{\infty} \otimes_{\mathrm{U}(1)} R_{\infty}^{*}$ is given by the $\mathrm{U}(1)$-invariant part of the tensor product matrix factorization $R_{\infty} \otimes R_{\infty}^{*}$. The U(1)-invariant generators of the latter are

$$
\begin{aligned}
g_{(i, j)} & :=f_{i} \otimes X^{n_{i}+\ldots+n_{j+1}-1} f_{j}^{*} & {[k+i,-k-1-j] } \\
g_{\left(d^{\prime}+i, j\right)} & :=f_{d^{\prime}+i} \otimes X^{n_{i}+\ldots+n_{j+1}-1} f_{j}^{*} & {[k+1+i,-k-1-j] } \\
g_{\left(i, d^{\prime}+j\right)} & :=f_{i} \otimes X^{n_{i}+\ldots+n_{j+1}-1} f_{d^{\prime}+j}^{*} & {[k+i,-k-j] } \\
g_{\left(d^{\prime}+i, d^{\prime}+j\right)} & :=f_{d^{\prime}+i} \otimes X^{n_{i}+\ldots+n_{j+1}-1} f_{d^{\prime}+j}^{*} & {[k+1+i,-k-j] }
\end{aligned}
$$

for $1 \leq i \leq d^{\prime}-1$ and $0 \leq j \leq i-1$. The $\mathbb{Z}_{d^{\prime}} \times \mathbb{Z}_{d^{\prime}}$-charges of the generators are specified in square brackets. Here, $f_{i}$ and $f_{i}^{*}$ label the generators of $R_{\infty}$ and $R_{\infty}^{*}$, respectively. The generators with $0 \leq i<d^{\prime}$ are $\mathbb{Z}_{2}$-even and the ones with $d^{\prime} \leq i<2 d^{\prime}$ are $\mathbb{Z}_{2}$-odd. Setting

$$
l=\frac{i(i+1)}{2}+j, \quad 0 \leq l \leq M:=\frac{\left(d^{\prime}+1\right)\left(d^{\prime}-2\right)}{2},
$$

one can order the generators as follows

$$
\begin{aligned}
g_{l} & =g_{(i, j)} \\
g_{M+l} & =g_{\left(d^{\prime}+i, d^{\prime}+j\right)} \\
g_{2 M+l} & =g_{\left(d^{\prime}+i, j\right)} \\
g_{3 M+l} & =g_{\left(i, d^{\prime}+j\right)} .
\end{aligned}
$$

$\left(R_{\infty} \otimes_{\mathrm{U}(1)} R_{\infty}^{*}\right)_{0}$ is then generated by the $g_{l}$ and $g_{M+l}$ for $0 \leq l \leq M$ and $\left(R_{\infty} \otimes_{\mathrm{U}(1)} R_{\infty}^{*}\right)_{1}$ by the $g_{2 M+l}$ and $g_{3 M+l}$ for $0 \leq l \leq M$.

In terms of the generators, the $\mathrm{U}(1)$-invariant tensor product matrix factorization

$$
\mathrm{d}=\mathrm{d}_{R} \otimes_{\mathrm{U}(1)} \mathbb{1}+\mathbb{1} \otimes_{\mathrm{U}(1)} \mathrm{d}_{R^{*}}=:\left(\begin{array}{c}
\mathrm{d}_{1} \\
\mathrm{~d}_{0}
\end{array}\right)
$$


takes the form

$$
\begin{aligned}
\left(\mathrm{d}_{1}\right)_{(p, q),\left(d^{\prime}+i, j\right)} & =\delta_{q, j}\left(Z \delta_{p, i}-\delta_{p, i+1}\right) \\
\left(\mathrm{d}_{1}\right)_{\left(d^{\prime}+p, d^{\prime}+q\right),\left(d^{\prime}+i, j\right)} & =\delta_{p, i} \theta(j-q) Y^{d^{\prime}-1-(j-q)} \\
\left(\mathrm{d}_{1}\right)_{(p, q),\left(i, d^{\prime}+j\right)} & =\delta_{p, i}\left(Y \delta_{q, j}-\delta_{q+1, j}\right) \\
\left(\mathrm{d}_{1}\right)_{\left(d^{\prime}+p, d^{\prime}+q\right),\left(i, d^{\prime}+j\right)} & =\delta_{q, j} \theta(p-i) Z^{d^{\prime}-1-(p-i)} \\
\left(\mathrm{d}_{0}\right)_{\left(d^{\prime}+i, j\right),(p, q)} & =\delta_{j, q} \theta(i-p) Z^{d^{\prime}-1-(i-p)} \\
\left(\mathrm{d}_{0}\right)_{\left(i, d^{\prime}+j\right),(p, q)} & =-\delta_{i, p} \theta(q-j) Y^{d^{\prime}-1-(q-j)} \\
\left(\mathrm{d}_{0}\right)_{\left(d^{\prime}+i, j\right),\left(d^{\prime}+p, d^{\prime}+q\right)} & =-\delta_{i, p}\left(Y \delta_{j, q}-\delta_{j+1, q}\right) \\
\left(\mathrm{d}_{0}\right)_{\left(i, d^{\prime}+j\right),\left(d^{\prime}+p, d^{\prime}+q\right)} & =\delta_{j, q}\left(Z \delta_{i, p}-\delta_{i, p+1}\right)
\end{aligned}
$$

where $1 \leq i, p \leq d^{\prime}, 0 \leq j<i, 0 \leq q<p$ and $\theta(x)=\left\{\begin{array}{l}1, x \geq 0 \\ 0, x<0\end{array}\right.$. For example, for $d^{\prime}=5$ one obtains

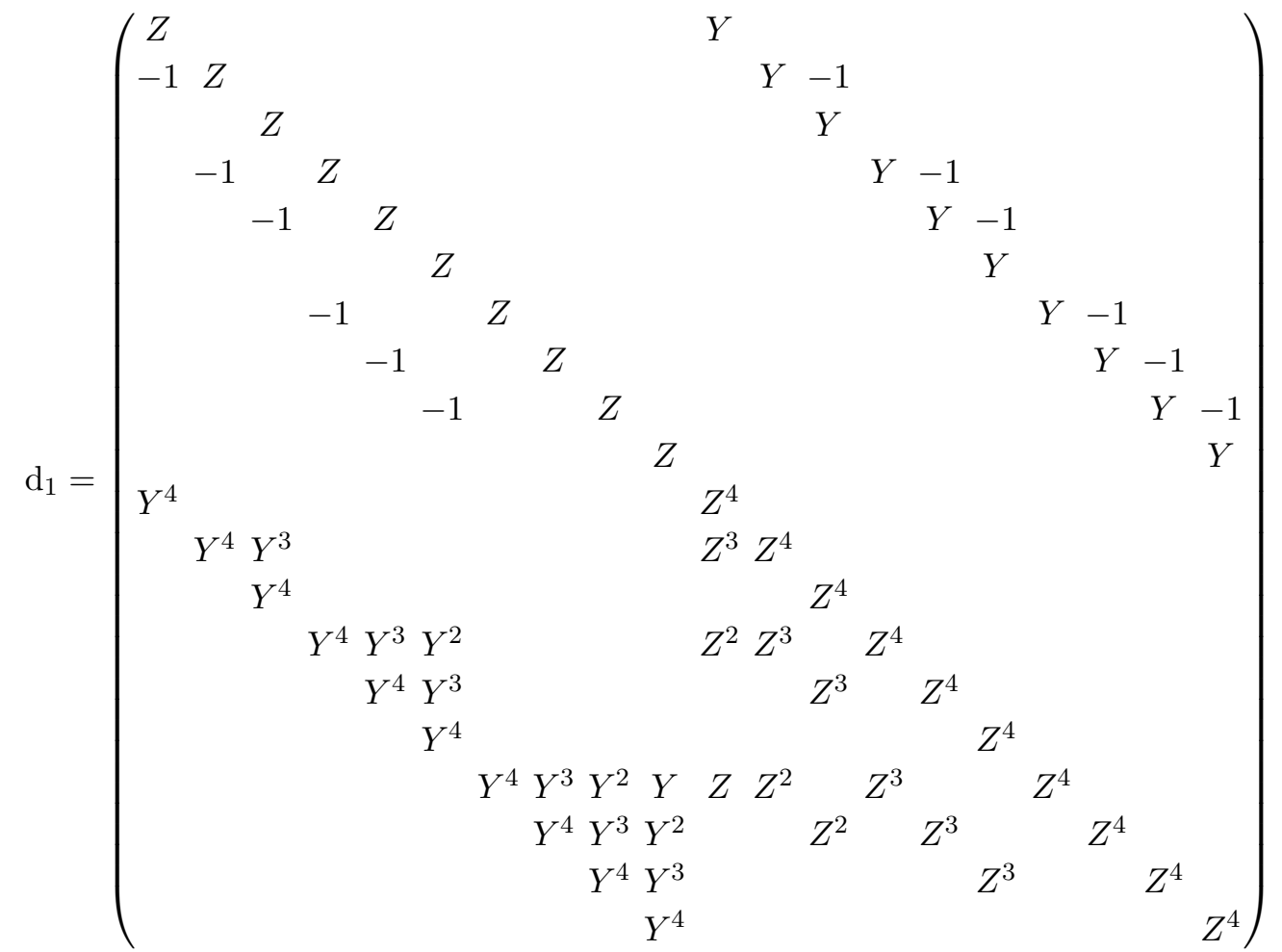

Stripping off trivial summands this matrix factorization reduces to the IR identity matrix factorization $\left(S^{\prime}=\mathbb{C}[Z, Y]\right)$

$$
I_{\mathrm{IR}}: S^{\prime d^{\prime}}\left(\begin{array}{c}
{[1,0]} \\
{[2,-1]} \\
{[3,-2]} \\
\vdots \\
{\left[d^{\prime},-d^{\prime}+1\right]}
\end{array}\right) \stackrel{\left(\begin{array}{ccccc}
Z & 0 & \ldots & 0 & -Y \\
-Y & Z & & & \\
0 & -Y & Z & & \\
\vdots & & \ddots & \ddots & \\
0 & & & -Y & Z
\end{array}\right)}{\rightleftarrows} S^{\prime d^{\prime}}\left(\begin{array}{c}
{[0,0]} \\
{[1,-1]} \\
{[2,-2]} \\
\vdots \\
\\
\end{array}\right.
$$


In order to see this, we perform a change of basis on (D.2):

$$
\mathrm{d}_{1}=S \cdot \tilde{\mathrm{d}}_{1} \cdot T^{-1}, \quad \mathrm{~d}_{0}=T \cdot \tilde{\mathrm{d}}_{0} \cdot S^{-1},
$$

where $S$ and $T^{-1}$ are defined by

$$
\begin{aligned}
(S)_{(p, q),(i, j)}= & \delta_{q, j}\left(\delta_{p, i}-Z \delta_{p+1, i}\right) \\
(S)_{(p, q),\left(d^{\prime}+i, d^{\prime}+j\right)}= & 0 \\
(S)_{\left(d^{\prime}+p, d^{\prime}+q\right),(i, j)}= & -\delta_{p+1, i} Y^{d^{\prime}-1-(j-q)} \theta(j-q) \\
& -\delta_{i, j+1}\left[Z^{d^{\prime}-p+i-1} Y^{q-i} \theta(q-i)+Z^{i-1-p} Y^{d^{\prime}+q-i} \theta(i-2-p)\right] \\
(S)_{\left(d^{\prime}+p, d^{\prime}+q\right),\left(d^{\prime}+i, d^{\prime}+j\right)}= & \delta_{q, j}\left(\delta_{p, i}+\theta(i-p-1) Z^{i-p}\right)+\delta_{i, d^{\prime}-1} \delta_{j, 0} \theta(q-1) Z^{d^{\prime}-1-p} Y^{q}
\end{aligned}
$$

and

$$
\begin{aligned}
\left(T^{-1}\right)_{\left(d^{\prime}+p, q\right),\left(d^{\prime}+i, j\right)}= & \delta_{q, j} \delta_{p, i} \\
& +\delta_{i, d^{\prime}-1}\left(-\delta_{q, j} Z^{d^{\prime}-1-p} \theta\left(d^{\prime}-1-p\right)+\delta_{p, d^{\prime}-1} Y^{q-j} \theta(q-j-1)\right) \\
\left(T^{-1}\right)_{\left(p, d^{\prime}+q\right),\left(d^{\prime}+i, j\right)}= & -\delta_{i, d^{\prime}-1} \delta_{q+1, p} \theta(q-j-1) Z^{d^{\prime}-1-q} Y^{q-1-j} \\
\left(T^{-1}\right)_{\left(d^{\prime}+p, q\right),\left(i, d^{\prime}+j\right)}= & -\delta_{q, j} Y\left(\delta_{p+1, i}+Z^{i-p-1} \theta(i-p-2)\right) \\
& \quad+\delta_{q+1, j} Z^{i-p-1} \theta(i-p-1) \\
\left(T^{-1}\right)_{\left(p, d^{\prime}+q\right),\left(i, d^{\prime}+j\right)}= & \delta_{p, i} \delta_{q, j}+\delta_{q, j} \delta_{q+1, p} \theta(i-p-1) Z^{i-p} .
\end{aligned}
$$

Here again $1 \leq i, p \leq d^{\prime}, 0 \leq j<i, 0 \leq q<p$. Then

$$
\begin{aligned}
\left(\tilde{\mathrm{d}}_{1}\right)_{(p, q),\left(d^{\prime}+i, j\right)} & =-\delta_{q, j} \delta_{p, i+1}+Z \delta_{i, d^{\prime}-1} \delta_{p, d^{\prime}-1} \delta_{q, d^{\prime}-2} \delta_{j, d^{\prime}-2} \\
\left(\tilde{\mathrm{d}}_{1}\right)_{\left(d^{\prime}+p, d^{\prime}+q\right),\left(d^{\prime}+i, j\right)} & =\delta_{p, d^{\prime}-1} \delta_{i, d^{\prime}-1}\left(W \delta_{q, j+1}-Y \delta_{q, 0} \delta_{j, d^{\prime}-2}\right) \\
\left(\tilde{\mathrm{d}}_{1}\right)_{(p, q),\left(i, d^{\prime}+j\right)} & =-Y \delta_{p, i} \delta_{q, j} \delta_{i, j+1}+Z \delta_{p+1, i} \delta_{p, j} \delta_{p, q+1} \\
\left(\tilde{\mathrm{d}}_{1}\right)_{\left(d^{\prime}+p, d^{\prime}+q\right),\left(i, d^{\prime}+j\right)} & =\delta_{i, p+1} \delta_{q, j} W+\delta_{i, 1} \delta_{j, 0} \delta_{p, d^{\prime}-1} \delta_{q, 0} Z
\end{aligned}
$$

and

$$
\begin{aligned}
&\left(\tilde{\mathrm{d}}_{0}\right)_{\left(d^{\prime}+i, j\right),(p, q)}=-\delta_{i+1, p} \delta_{j, q} W+\delta_{i, d^{\prime}-1} \delta_{j, d^{\prime}-2} \delta_{p, q+1} Z^{p} Y^{d^{\prime}-1-p} \\
&\left(\tilde{\mathrm{d}}_{0}\right)_{\left(i, d^{\prime}+j\right),(p, q)}=-\delta_{i, j+1} \delta_{p, q+1}\left(\theta(p-i) Y^{d^{\prime}-1-p+i} Z^{p-i}\right. \\
&\left.+\theta(i-p-1) Z^{d^{\prime}-i+p} Y^{i-p-1}\right) \\
&\left(\tilde{\mathrm{d}}_{0}\right)_{\left(d^{\prime}+i, j\right),\left(d^{\prime}+p, d^{\prime}+q\right)}=\delta_{p, d^{\prime}-1} \delta_{i, d^{\prime}-1}\left(\delta_{j+1, q}-Y^{d^{\prime}-1} \delta_{j, d^{\prime}-2} \delta_{q, 0}\right) \\
&\left(\tilde{\mathrm{d}}_{0}\right)_{\left(i, d^{\prime}+j\right),\left(d^{\prime}+p, d^{\prime}+q\right)}=-\delta_{i, p+1} \delta_{j, q}+\delta_{p, d^{\prime}-1} \delta_{q, 0} \delta_{i, j+1} Z^{d^{\prime}-i} Y^{j}
\end{aligned}
$$

are matrix factorization of $W=Z^{d^{\prime}}-Y^{d^{\prime}}$ which reduce to the indentity matrix factorization. 
In the example $d^{\prime}=5 \mathrm{~d}_{1}$ above turns into

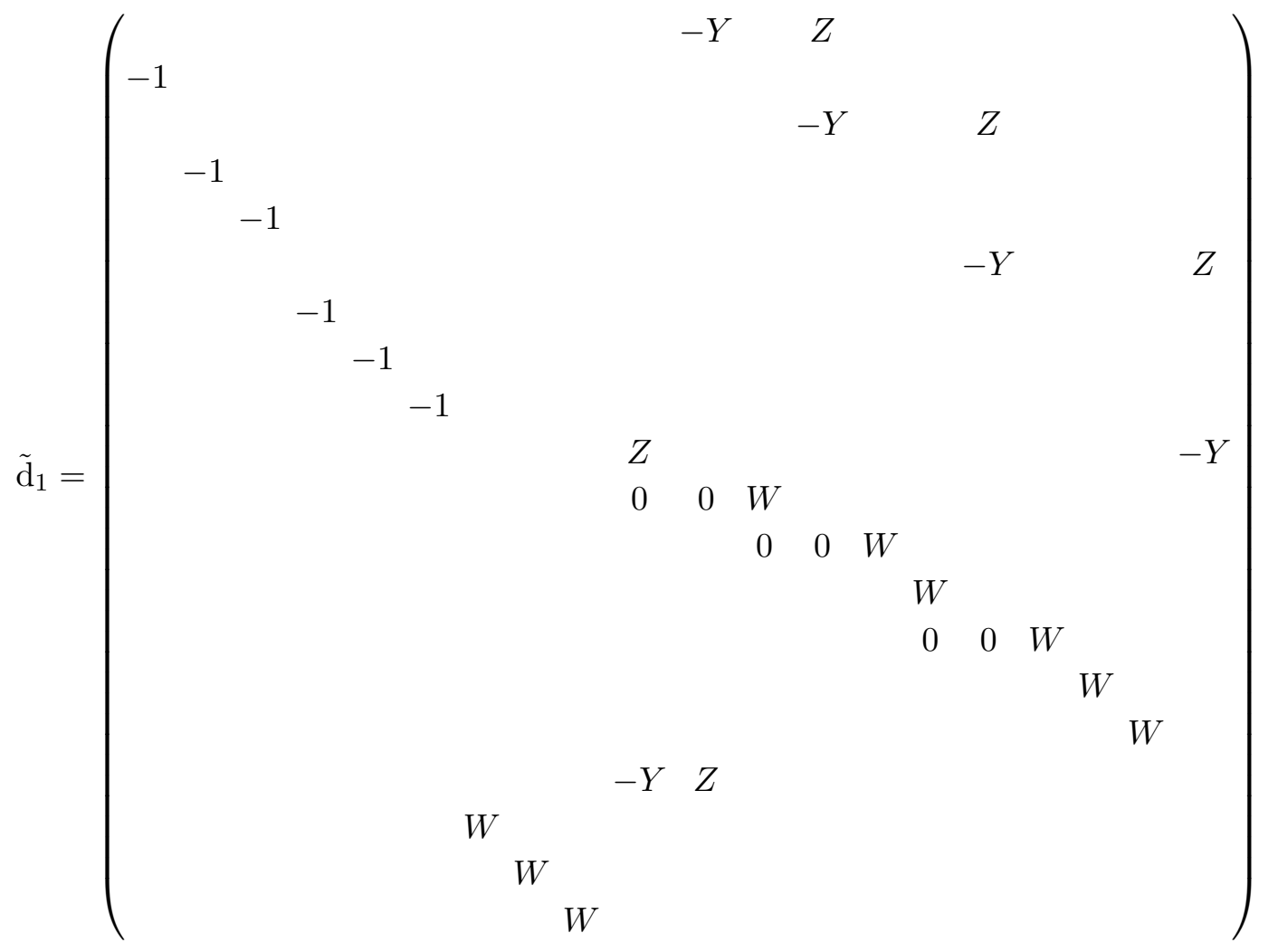

which is easily recognized as the matrix associated to a sum of the identity matrix factorization $I_{\mathrm{IR}}$ with a number of trivial rank-one matrix factorizations.

In the general case, the generators not belonging to trivial summands are the ones labelled by the restricted index sets

$$
\begin{aligned}
\{(i, j) \mid i=j+1\} & \subset\left\{(i, j) \mid i=1, \ldots, d^{\prime}-1 ; j=0, \ldots, i-1\right\} \\
\left\{\left(d^{\prime}+i, d^{\prime}+j\right) \mid i=d^{\prime}-1, j=0\right\} & \subset\left\{\left(d^{\prime}+i, d^{\prime}+j\right) \mid i=1, \ldots, d^{\prime}-1 ; j=0, \ldots, i-1\right\} \\
\left\{\left(d^{\prime}+i, j\right) \mid i=d^{\prime}-1, j=d^{\prime}-2\right\} & \subset\left\{\left(d^{\prime}+i, j\right) \mid i=1, \ldots, d^{\prime}-1 ; j=0, \ldots, i-1\right\} \\
\left\{\left(i, d^{\prime}+j\right) \mid i=j+1\right\} & \subset\left\{\left(i, d^{\prime}+j\right) \mid i=1, \ldots, d^{\prime}-1 ; j=0, \ldots, i-1\right\}
\end{aligned}
$$

Restricting to these generators yields the IR identity defect $I_{\mathrm{IR}}$.

Open Access. This article is distributed under the terms of the Creative Commons Attribution License (CC-BY 4.0), which permits any use, distribution and reproduction in any medium, provided the original author(s) and source are credited.

\section{References}

[1] I. Brunner and D. Roggenkamp, Defects and bulk perturbations of boundary Landau-Ginzburg orbifolds, JHEP 04 (2008) 001 [arXiv: 0712.0188] [INSPIRE].

[2] D. Gaiotto, Domain Walls for Two-Dimensional Renormalization Group Flows, JHEP 12 (2012) 103 [arXiv:1201.0767] [INSPIRE]. 
[3] J. Fröhlich, J. Fuchs, I. Runkel and C. Schweigert, Duality and defects in rational conformal field theory, Nucl. Phys. B 763 (2007) 354 [hep-th/0607247] [InSPIRE].

[4] J. Fröhlich, J. Fuchs, I. Runkel and C. Schweigert, Defect lines, dualities and generalised orbifolds, in Proceedings, 16th International Congress on Mathematical Physics (ICMP09): Prague, Czech Republic, August 3-8, 2009, 2009, DOI [arXiv:0909.5013] [INSPIRE].

[5] N. Carqueville and I. Runkel, Orbifold completion of defect bicategories, Quantum Topol. 7 (2016) 203 [arXiv: 1210.6363] [INSPIRE].

[6] N. Carqueville, A.R. Camacho and I. Runkel, Orbifold equivalent potentials, J. Pure Appl. Algebra 220 (2016) 759 [arXiv:1311.3354] [INSPIRE].

[7] I. Brunner, N. Carqueville and D. Plencner, A quick guide to defect orbifolds, Proc. Symp. Pure Math. 88 (2014) 231 [arXiv:1310.0062] [INSPIRE].

[8] I. Brunner, N. Carqueville and D. Plencner, Orbifolds and topological defects, Commun. Math. Phys. 332 (2014) 669 [arXiv:1307.3141] [INSPIRE].

[9] A. Kapustin, Topological Field Theory, Higher Categories and Their Applications, in International Congress of Mathematicians (ICM 2010) Hyderabad, India, August 19-27, 2010, arXiv: 1004.2307 [INSPIRE].

[10] A. Davydov, L. Kong and I. Runkel, Field theories with defects and the centre functor, arXiv: 1107.0495 [INSPIRE].

[11] N. Carqueville, Lecture notes on 2-dimensional defect TQFT, arXiv:1607.05747 [INSPIRE].

[12] N. Carqueville and D. Murfet, Adjunctions and defects in Landau-Ginzburg models, Adv. Math. 289 (2016) 480 [arXiv: 1208.1481] [INSPIRE].

[13] I. Brunner and D. Roggenkamp, B-type defects in Landau-Ginzburg models, JHEP 08 (2007) 093 [arXiv: 0707.0922] [INSPIRE].

[14] I. Brunner, N. Carqueville and D. Plencner, Discrete torsion defects, Commun. Math. Phys. 337 (2015) 429 [arXiv: 1404.7497] [INSPIRE].

[15] J. Fuchs, I. Runkel and C. Schweigert, TFT construction of RCFT correlators 1. Partition functions, Nucl. Phys. B 646 (2002) 353 [hep-th/0204148] [INSPIRE].

[16] A. Kapustin and Y. Li, D branes in Landau-Ginzburg models and algebraic geometry, JHEP 12 (2003) 005 [hep-th/0210296] [INSPIRE].

[17] I. Brunner, M. Herbst, W. Lerche and B. Scheuner, Landau-Ginzburg realization of open string TFT, JHEP 11 (2006) 043 [hep-th/0305133] [INSPIRE].

[18] M. Khovanov and L. Rozansky, Topological Landau-Ginzburg models on a world-sheet foam, Adv. Theor. Math. Phys. 11 (2007) 233 [hep-th/0404189] [INSPIRE].

[19] D. Orlov, Triangulated categories of singularities and D-branes in Landau-Ginzburg models, Trudy Steklov Mat. Inst. 246 (2004) 240 [math/0302304] [INSPIRE].

[20] N. Carqueville and I. Runkel, Rigidity and defect actions in Landau-Ginzburg models, Commun. Math. Phys. 310 (2012) 135 [arXiv:1006.5609] [INSPIRE].

[21] N. Carqueville and D. Murfet, A toolkit for defect computations in Landau-Ginzburg models, Proc. Symp. Pure Math. 90 (2015) 239 [arXiv:1303.1389] [INSPIRE].

[22] K.A. Intriligator and C. Vafa, Landau-Ginzburg ORBIFOLDS, Nucl. Phys. B 339 (1990) 95 [INSPIRE]. 
[23] S.K. Ashok, E. Dell'Aquila and D.-E. Diaconescu, Fractional branes in Landau-Ginzburg orbifolds, Adv. Theor. Math. Phys. 8 (2004) 461 [hep-th/0401135] [InSPIRE].

[24] K. Hori and J. Walcher, F-term equations near Gepner points, JHEP 01 (2005) 008 [hep-th/0404196] [INSPIRE].

[25] M. Becker, Y. Cabrera and D. Robbins, Defects and boundary $R G$ flows in $\mathbb{C} / \mathbb{Z}_{d}$, JHEP 02 (2017) 007 [arXiv: 1611.01133] [INSPIRE].

[26] C. Vafa, Mirror symmetry and closed string tachyon condensation, hep-th/0111051 [INSPIRE].

[27] G.W. Moore and A. Parnachev, Profiling the brane drain in a nonsupersymmetric orbifold, JHEP 01 (2006) 024 [hep-th/0507190] [INSPIRE].

[28] G.W. Moore and A. Parnachev, Localized tachyons and the quantum McKay correspondence, JHEP 11 (2004) 086 [hep-th/0403016] [INSPIRE].

[29] I. Brunner, D. Roggenkamp and S. Rossi, Defect Perturbations in Landau-Ginzburg Models, JHEP 03 (2010) 015 [arXiv: 0909.0696] [INSPIRE].

[30] S. Cecotti and C. Vafa, Topological-antitopological-fusion, Nucl. Phys. B 367 (1991) 359 [INSPIRE].

[31] S. Cecotti and C. Vafa, On classification of $N=2$ supersymmetric theories, Commun. Math. Phys. 158 (1993) 569 [hep-th/9211097] [INSPIRE].

[32] C. Bachas and I. Brunner, Fusion of conformal interfaces, JHEP 02 (2008) 085 [arXiv:0712.0076] [INSPIRE].

[33] C. Bachas, I. Brunner and D. Roggenkamp, A worldsheet extension of $O(d, d \mid Z)$, JHEP 10 (2012) 039 [arXiv: 1205.4647] [INSPIRE].

[34] V.V. Bazhanov, S.L. Lukyanov and A.B. Zamolodchikov, Integrable structure of conformal field theory, quantum KdV theory and thermodynamic Bethe ansatz, Commun. Math. Phys. 177 (1996) 381 [hep-th/9412229] [INSPIRE].

[35] V.V. Bazhanov, S.L. Lukyanov and A.B. Zamolodchikov, Integrable structure of conformal field theory. 2. Q operator and DDV equation, Commun. Math. Phys. 190 (1997) 247 [hep-th/9604044] [INSPIRE].

[36] V.V. Bazhanov, S.L. Lukyanov and A.B. Zamolodchikov, Integrable structure of conformal field theory. 3. The Yang-Baxter relation, Commun. Math. Phys. 200 (1999) 297 [hep-th/9805008] [INSPIRE].

[37] I. Runkel, Perturbed Defects and T-Systems in Conformal Field Theory, J. Phys. A 41 (2008) 105401 [arXiv:0711.0102] [INSPIRE].

[38] I. Runkel, Non-local conserved charges from defects in perturbed conformal field theory, J. Phys. A 43 (2010) 365206 [arXiv: 1004.1909] [inSPIRE].

[39] N. Carqueville, I. Runkel and G. Schaumann, Orbifolds of n-dimensional defect TQFTs, Geom. Topol. 23 (2019) 781 [arXiv:1705. 06085] [INSPIRE].

[40] J. Fuchs and C. Stigner, On frobenius algebras in rigid monoidal categories, Arab. J. Sci. Eng. 33-2C (2008) 175 [arXiv:0901.4886].

[41] D. Eisenbud, Homological algebra on a complete intersection, with an application to group representations, Trans. Am. Math. Soc. 260 (1980) 35. 